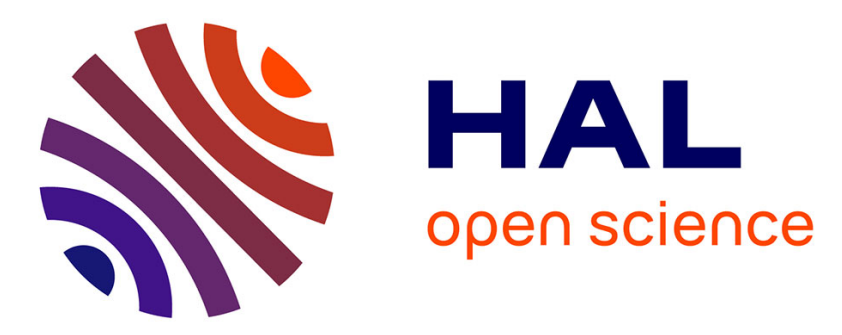

\title{
Hard x-ray spectroscopy and dynamics of isolated atoms and molecules: a review
}

\author{
M. Piancastelli, T. Marchenko, R. Guillemin, L. Journel, O. Travnikova, I. \\ Ismail, M. Simon
}

\section{- To cite this version:}

M. Piancastelli, T. Marchenko, R. Guillemin, L. Journel, O. Travnikova, et al.. Hard x-ray spectroscopy and dynamics of isolated atoms and molecules: a review. Reports on Progress in Physics, 2020, 83 (1), pp.016401. 10.1088/1361-6633/ab5516 . hal-02884031

\section{HAL Id: hal-02884031 \\ https://hal.sorbonne-universite.fr/hal-02884031}

Submitted on 29 Jun 2020

HAL is a multi-disciplinary open access archive for the deposit and dissemination of scientific research documents, whether they are published or not. The documents may come from teaching and research institutions in France or abroad, or from public or private research centers.
L'archive ouverte pluridisciplinaire HAL, est destinée au dépôt et à la diffusion de documents scientifiques de niveau recherche, publiés ou non, émanant des établissements d'enseignement et de recherche français ou étrangers, des laboratoires publics ou privés. 


\title{
Hard x-ray spectroscopy and dynamics of isolated atoms and molecules: a review
}

\author{
M. N. Piancastelli ${ }^{1,2, *}$ T. Marchenko ${ }^{1}$, R. Guillemin ${ }^{1}$, L. Journel ${ }^{1}$, O. Travnikova ${ }^{1}$, I. Ismail ${ }^{1}$ \\ and M. Simon ${ }^{1}$ \\ ${ }^{1}$ Sorbonne Université, CNRS, Laboratoire de Chimie Physique-Matière et Rayonnement, \\ LCPMR, F-75005 Paris, France \\ ${ }^{2}$ Department of Physics and Astronomy, Uppsala University, SE-75120 Uppsala, Sweden
}

maria-novella.piancastelli@physics.uu.se

\begin{abstract}
$\underline{\text { Abstract }}$
We present here a review of the most significant recent achievements in the field of HAXPES (HArd X-ray PhotoElectron Spectroscopy) on isolated atoms and molecules, and related spectroscopies. The possibility of conducting hard $\mathrm{x}$-ray photoexcitation and photoionization experiments under state-of-the art conditions in terms of photon and electron kinetic energy resolution has become available only in the last few years. HAXPES has then produced structural and dynamical information at the level of detail already reached in the VUV and soft-x-ray ranges. The much improved experimental conditions have allowed extending to the hard $\mathrm{x}$-ray range some methods well established in soft $\mathrm{x}$-ray spectroscopies. Investigations of electron and nuclear dynamics in the femtosecond (fs, $10^{-15} \mathrm{sec}$ ) and even attosecond (as, $10^{-18} \mathrm{sec}$ ) regime have become feasible. Complex relaxation phenomena following deep-core ionization can now be enlightened in great detail. Other phenomena like e.g. recoil-induced effects are much more important in fast photoelectron emission, which can be induced by hard x-rays. Furthermore, a new kind of ionic states with double core holes can be observed by x-ray single-photon absorption. Future perspectives are also discussed.
\end{abstract}




\section{Contents:}

1. Introduction

2. Background

2.1 Auger resonant Raman

2.2 Core-hole-clock

2.3 Effective scattering time

2.4 Post-collision interaction

2.5 Cascade Auger decay

2.6 Double-core-hole studies

3. Instrumentation

3.1 Beam lines

3.2 Spectrometers

4. Recent results

4.1 RIXS: reaching the attosecond timescale

4.2 Double-core-hole studies by single-channel photoemission

4.3 Direct derivation of potential energy surfaces

4.4 Ultrafast photodissociation in the Auger cascade following deep-core excitation

4.5 Recoil due to the photoelectron's momentum

4.6 Exotic Auger decay pathways

4.7 Novel interference phenomena

4.8 Electron-recapture and post-collision interaction effects

4.9 Disentangling complex decay patterns after deep-core-hole ionization

4.10 Core-hole localization/delocalization

5. Perspectives

6. Conclusion

7. Acknowledgments

8. Literature references 


\section{Introduction}

The interaction between light and matter in general, and more specifically the photoelectric effect, has been among the foundations of the development of physics in the last century. Einstein's paper in 1905 [1] is one of the pillars not only of modern spectroscopy, but, even more, of our comprehension of the nature of electromagnetic radiation and its interaction with matter.

The technique based on such effect, photoelectron spectroscopy, is nowadays one of the most powerful tools at our disposal to investigate the electronic structure of atoms, molecules, liquids and solids. Its main capability is to measure the kinetic energy of photoemitted electrons, and, knowing the wavelength of the ionizing radiation, derive from there the valence and core orbital structure. Combined with suitable theoretical calculations, photoelectron spectroscopy gives a unique picture of the occupied levels of the system under investigation.

In a very general framework, the study of the electronic structure and dynamics of a system can be performed by a combination of spectroscopic tools. While photoelectron spectroscopy is the technique of choice to investigate filled electronic levels, other complementary techniques offer the possibility to look at empty states and at the processes which can follow photoelectron emission. The photoabsorption cross section near an inner-shell orbital can be measured, which provides information on unoccupied electronic states just below the ionization threshold, and to the local structure around the photoexcited atom. The inner-shell electron vacancy can be subsequently occupied by a valence electron (e.g., from a highest occupied molecular orbital state, HOMO), and either photons or electrons can be emitted in this decay process. The related techniques are x-ray emission (collecting the emitted photons) or Auger spectroscopy (collecting the emitted electrons). A combination of such spectroscopies gives a complete picture of the electronic structure and dynamics of atoms, molecules, liquids and solids.

In this review we will deal mainly with photoemission from deep electronic levels and the subsequent relaxation processes after an inner-shell vacancy is created. Our group is also using resonant $\mathrm{x}$-ray emission spectroscopy and we will show some related examples.

The photon energy sources traditionally used in photoelectron spectroscopy experiments are ultraviolet or x-ray sources, depending on whether valence or core levels are mainly investigated. While laboratory sources were used at the beginning of the technique, synchrotron radiation has proved to be the most versatile tool, due to its tunability in a large range.

The high-energy version of photoelectron spectroscopy, now usually called hard-x-ray photoelectron spectroscopy or HAXPES, applied to isolated atoms and molecules, is the main topic of this review article. We specify immediately that we will consider the photon energy range from 2 to $12 \mathrm{keV}$, which is perhaps lower that the usual hard $\mathrm{x}$-ray energy interval (from $5 \mathrm{keV}$ up). We often call it "tender" $\mathrm{x}$-ray domain, to underline that it is somewhat inbetween soft and hard. However, the majority of new studies in atomic and molecular physics, which are our main subject, are conducted in this "tender" energy range rather than with harder X-rays.

A general overview of the results obtained in this newly accessible photon energy range is the following: deeper and deeper electronic levels reached by photoionization; shorter and shorter lifetimes of the states produced this way, which allows studying electron and nuclear dynamics on the timescale of femtoseconds ( $\mathrm{fs}, 10^{-15} \mathrm{~s}$ ) and even attoseconds (as, $10^{-18} \mathrm{~s}$ ); deeper and deeper core electron emission leads to faster and faster Auger electron, shorter and shorter core hole lifetime as well as higher and higher charge of the produced ions due to the cascade Auger effect; strong interaction between the photoelectron, the Auger electron 
and the ion left behind; investigation of physical phenomena well-known in the macroscopic world such as recoil and Doppler effects but occurring at the atomic and molecular level; identification of molecular dynamics on the several relaxation steps in cascade following deep core ionization and excitation; characterization of new electronic states with multiple vacancies exhibiting large chemical shifts; electronic states with highly repulsive potential energy surfaces leading to ultrafast nuclear dynamics; novel phenomena such as electronic state/lifetime interference which are typical of this new regime; possibility of investigating Compton scattering in a suitable photon energy range under state-of-the-art experimental conditions for both electron and photon detection.

Beside the interest of $x$-ray spectroscopies in fundamental research, we can point out that, in general, $\mathrm{x}$-rays are widely used in medicine, both for diagnostics and for treatment (radiation therapy), as well as in other scientific fields, e.g. in crystallography. In all these uses, radiation damage induced by ionization occurs, in human tissues and biomaterials.

The first steps in radiation damage involve $\mathrm{x}$-rays ionizing deep core levels, typically in high$\mathrm{Z}$ elements. Such ionized species with a deep electron vacancy are unstable and rapidly decay, often in cascades, via both radiative (photon emission) and non-radiative (Auger electron emission) channels. The Auger decay involves the emission of electrons, and the cascades result in increasingly positively charged ions. These processes lead to extensive local ionization, and eventually to bond breaking and formation of radicals, which are important for radiation damage in biological systems. While radiation damage constitutes a hazard in medical diagnostics, it may also be used, e.g. in tumour radiation therapy.

How the photon energy primarily absorbed by the high- $Z$ atom is dissipated into several nuclear and electronic degrees of freedom, and to what extent this can be used to improve the therapeutic efficiency, is an open question, which requires detailed knowledge of the first steps towards radiation damage, i.e. the x-ray-induced electronic and nuclear dynamics. The results we will show in this work clearly demonstrate that the newly accessible extended photon energy range does not simply allow studying more systems with deeper core edges, but opens a totally new horizon in what concerns electron and nuclear dynamics of deep coreexcited and core-ionized isolated species. A profound knowledge of such decay processes is very important not only in the assessment of radiation damage in biosystems, but also in plasma physics and astrophysics, and in many other fields outside physics.

Back to HAXPES, we can date the start of its history to the middle of last century, with the implementation of its ancestor, Electron Spectroscopy for Chemical Analysis (ESCA) [2,3]. This technique uses x-rays (typically $1486.6 \mathrm{eV}$ with an aluminum anode as source) and single-photon absorption, leading to the emission of one electron from a deep shell. By definition, the energy necessary to extract an electron, the ionization energy, is equal to the energy which holds the electron in the system, the binding energy.

The two key points of the ESCA technique were: 1) the possibility to identify the atomic species present in a solid or gaseous sample, since the binding energy of core electrons is typical of a specific atom, and therefore to derive the chemical composition of the sample; and 2) the fact that, depending upon the chemical environment of an atom in a molecule or a solid, its binding energy is slightly different (the well-known "chemical shift").

In addition to measuring photoelectrons, another asset of by ESCA was to follow the evolution of a system with a core vacancy. In particular, the relaxation via Auger electron emission (one shallower electron filling the hole, and another shallower electron being emitted in the continuum) was also possible to measure.

Few decades after the development of the ESCA technique, a great impulse to studies of core photoelectron spectroscopy of atoms, molecules and solid has been given by the advent of synchrotron radiation as ionizing source. Compared to laboratory sources, synchrotron 
radiation offers the great advantages of tunability, intensity and stability. Studies performed with a tunable source have some important advantages with respect to single-wavelength laboratory sources, the first being the possibility to choose a specific energy to selectively photoexcite the systems under investigation by promoting a core or valence electron to a reachable empty level, and then follow the dynamics of the intermediate neutral excited state created in this way. This particular way is called resonant photoemission. However, apart from few significant examples (see chapter 2), the focus in gas-phase studies for the past few decades has been in the VUV and soft-x-ray regions of the spectrum rather than hard x-rays. While hard x-ray spectroscopy has been performed with rather limited experimental tools since decades, the possibility of conducting experiments under state-of-the art conditions in terms of photon and electron kinetic energy resolution is very recent, namely in the last five or six years. Since then, HAXPES of atoms and molecules has been able to produce very detailed structural and dynamical information at the level already reached in the VUV and soft-x-ray ranges.

In particular, the much improved experimental conditions have allowed extending to the hard $\mathrm{x}$-ray range some methods well established in soft $\mathrm{x}$-ray spectroscopies, such as the core-hole clock method [4-6]. Since for deep core vacancies the core-hole lifetime before relaxation via Auger or x-ray emission decay is much shorter than for shallower holes, electron and nuclear dynamics in the femtosecond (fs, $10^{-15} \mathrm{sec}$ ) and even attosecond (as, $10^{-18} \mathrm{sec}$ ) regime can be studied. Relaxation phenomena following deep-core ionization imply a cascade of events which can now be enlightened in great detail. Other phenomena like e.g. recoil-induced effects are much more important in fast photoelectron emission, which can be induced by hard x-rays. Furthermore, a new kind of ionic states with double core holes can be observed by $\mathrm{x}$-ray single-photon absorption.

The relaxation process of a deep core vacancy can imply multiple steps, where an interplay of radiative (photon emission) and nonradiative (Auger electron emission) decay patterns can be observed. Therefore complementary techniques to study electron and nuclear dynamics following deep core excitation and ionization are Auger electron emission, x-ray emission and RIXS (Resonant Inelastic X-ray Scattering).

RIXS is well established in spectroscopy of isolated atoms and molecules. It exhibits remarkable sensitivity as a probe of the chemical environment of a specific atom in a molecule, and can also shed light on dynamical aspects such as nuclear dynamics in a molecule following core-hole excitation. It has recently been applied in the hard $\mathrm{x}$-ray domain to molecular systems containing heavy atoms such as $\mathrm{Cl}$ (K-shell) and I (L-shell). As an example, nonlinear dispersion and a significant narrowing of inelastic emission lines around the resonant excitation were demonstrated to reflect molecular bond elongation after deep-core excitation in $\mathrm{HCl}$ [7]. There the sensitivity of RIXS spectral features to the nuclear motion on a sub-Ångström scale in the time interval set by the natural lifetime of $1 \mathrm{fs}$ was exploited. In our subsequent work on deep core excitation, we have shown that by RIXS we can have access to the subfemtosecond timescale, and even reach the attosecond regime.

Together with high-resolution photoelectron, Auger and x-ray emission spectroscopies, another mainstream in research on photoionization and photoexcitation dynamics of isolated species is the use of coincidence techniques, meaning in general the detection of two or more charged particles from the same ionization event. To obtain a complete picture of a photoinduced process such as the photoexcitation of a bound electron to a virtual state or the photoionization of a core or valence electron, a simultaneous investigation of all emitted particles is needed to shed light on the full dynamics of photon-molecule impact and of the subsequent decay pathways, which include electron emission and fragmentation of the molecular ion. 
In particular, the simultaneous detection of one electron and one or two ion fragments allows one to connect a specific electronic decay process to a specific ion production in general and to a specific fragmentation pattern in molecules. Throughout the last 15 years, the most used techniques have been PEPICO (photoelectron-photoion coincidence) and PEPIPICO (photoelectron-photoion-photoion coincidence) (for reviews of earlier work, see [8,9]).

More recently, a new technique, called COLTRIMS (COLd Target Recoil Ion Momentum Spectroscopy) [10], has been developed which makes the simultaneous collection of all charged fragments possible. In a COLTRIMS apparatus, there are two spectrometers, one for ion detection and one for electron detection, which in principle allow $4 \pi$ collection of all charged particles. Momenta of electrons and ions can then be calculated from their flight times and their impact locations on the detectors. This novel imaging technique allows coincident determination of the momentum vectors of particles from ionizing interactions of photons with atoms or molecules. After data collection, the momentum space can be analyzed along any particular direction (e.g., to select electron-ion coincidences).

While COLTRIMS has been used up to few years ago exclusively in the soft x-ray range, a new apparatus of such family has been recently developed by our group and installed at SOLEIL to perform similar measurements in the tender x-ray range. A closer look has been possible on phenomena such as post-collision interaction and core-hole localizationdelocalization dynamics.

While the HAXPES research area is based on the new opportunities offered at synchrotron sources, an important aspect is also its complementarity with the new x-ray free-electron lasers (FELs). As an example, the new scientific area concerning the study of double core holes created in isolated atoms and molecules has been carried out both at the LCLS FEL facility and at SOLEIL, providing a synergy between spectroscopy and dynamics of such novel final states which can be reached through very different pathways (see Sections 2.6 and 4.2). Another important complementarity between synchrotron radiation sources and $x$-ray FELs is that up to now COLTRIMS-like techniques were not possible to implement at FELs, while their use has been successful in HAXPES at synchrotrons.

Another complementarity worth mentioning is between HAXPES in gas phase and in solid state. The higher resolution available for isolated species can be very useful to disentangle solid-state-related effects from purely atomic and molecular ones. As an example, recoil effects at have been measured in gas phase (see Section 4.5), and can be present in solids as well, giving rise to broadening in Auger peak widths at higher energies.

Here we will review the current status of research in atomic and molecular physics by HAXPES and electron-ion coincidence techniques in the tender x-ray regime. The main activity has been carried out at the French synchrotron radiation facility SOLEIL near Paris. We wish to point out that up to now our team has been the main player in this new field, therefore our selection is based on our most significant papers. However, following this lead, new experimental programs are under rapid development at SPring-8 in Japan, MAX IV in Sweden, APS near Chicago, and PETRA III in Hamburg, Germany. Furthermore, the HAXPES conference series is well established (see [11] for a history of the conference).

The outline of this review is the following: in chapter 2 we will summarize the results obtained before the latest instrumental developments, and the concepts and methods already used in the soft $\mathrm{x}$-ray range which are further developed to adapt them to the harder $\mathrm{x}$-ray range; in chapter 3 we will describe the instrumentation which made possible the recent advances (beam lines and end stations at synchrotrons SOLEIL and ESRF); and in chapter 4 we will describe the latest results, ordered by topic. We will then proceed with some perspectives (chapter 5) and conclusion (chapter 6). 


\section{Background}

We have published few years ago a comprehensive book chapter on HAXPES at synchrotron radiation facilities in its early stage [12]. Therefore we will present in this section only some highlights of the development of the field prior to the advent of the recent instrumental advances. We will concentrate here on some significant earlier experimental results. Furthermore, we will introduce here some important concepts and tools developed for the soft $\mathrm{x}$-ray range which are at the foundation of the latest developments we will discuss in the rest of the review.

\subsection{Auger resonant Raman effect}

The first HAXPES study which can be considered as a major breakthrough was published in 1980 [13]. It reports Xe resonant photoemission around the 2p edge. The key point is that it demonstrated the Auger resonant Raman effect, which has been used in many publications since then. The experimental evidence is the following: after photoexcitation of Xe $2 p$ electrons to $5 \mathrm{~d}$, the system relaxes by emitting a valence electron while another valence electron fills the hole. This is the equivalent of Auger emission, only after resonant excitation, so it is called resonant Auger decay. The definition of Auger resonant Raman is the following: one observes linear dispersion of the kinetic energy of the resonant Auger spectral features in the HAXPES spectra as a function of photon energy, and narrowing of the resonant-Auger peaks when the photon energy is tuned to the maximum of the resonance.

The physics behind is the following. The linear dispersion of the resonant-Auger peaks is a consequence of energy conservation: the ejected electron "remembers" the excitation photon energy value, and, if the experimental conditions allow one to vary such energy within the width of the resonance, then the resonant-Auger peaks disperse accordingly. The line narrowing derives from the fact that the width of the resonant-Auger peaks reflects the width of the exciting radiation, rather than the natural lifetime width of the core hole.

This firs result has opened the way to a series of works in which the photon energy has been scanned through a resonance to observe the decay dynamics.

\subsection{Core-hole-clock}

Another milestone in earlier research is the development of the so-called core-hole clock method [4-6]. This method has been first established theoretically and then applied experimentally in the soft x-ray range, but it has important recent developments in HAXPES. The heart of the method is the following: if a core electron is emitted, the ion with a deep vacancy will be unstable, and decay by electron or photon emission. The time the core hole survives depends upon its location and the nature of the atom where the vacancy is created. The K-edge core-hole lifetime ranges between few femtoseconds for light elements to 1 femtosecond for third-row elements, and can reach the attosecond timescale for deep holes in heavy elements. The core-hole lifetime $\tau$ can be derived by the width $\Delta \mathrm{E}$ of the corresponding spectral feature in the photoelectron spectrum, via the equivalent of the Heisenberg principle in the time-energy domain:

$$
\Delta E \Delta \tau \geq \frac{\hbar}{2}
$$


The core-hole-clock method uses the decay time of a core excitation as a reference clock for other processes in the system studied. Typically, the decay of a core excitation yields a spectral signature that is characteristic for a given system. If other processes are taking place during the core-hole decay, they will also have their own identifiable spectral signature. The core-hole lifetime can then be used as "internal clock" to study some dynamical phenomena. The most widely studied one is the so-called ultrafast fragmentation, meaning molecular fragmentation taking place on the same timescale of the core-hole decay. If nuclear motion in a photoexcited molecule leads to the breaking of a chemical bond before electronic relaxation, the decay takes place in the fragment rather than in the intact molecule. The spectrum of the fragment will then be superimposed to the one of the intact molecule, and will be possible to assign [4, 14-20].

\subsection{Effective scattering time}

Another key concept introduced in works in the soft x-ray range is the possibility to actually manipulate the excitation-deexcitation processes by the so-called detuning procedure, meaning the detuning of the excitation energy from the maximum of the photoabsorption resonance. The so-called "scattering time" $\tau_{\text {eff }}$ is introduced, defined as [21]:

$$
\left.\tau_{\mathrm{eff}} \cong 1 / \sqrt{(} \Omega^{2}+\Gamma^{2}\right)
$$

where $\Gamma$ is the lifetime broadening and $\Omega$ is the detuning, or the distance in energy from the top of the resonance. The larger is the mismatch (i.e. detuning, $\Omega$ ) between the chosen photon energy and the vertical absorption energy, the shorter the scattering time $\tau_{\text {eff }}$ due to the increased dephasing of the wave packet [22]. By controlling the effective scattering time, some properties of the systems can be influenced, as e.g. the ratio of molecular-versusfragment electron decays, and in general the evolution of the wave packet formed by the excitation.

\subsection{Post-collision interaction and recapture}

To continue with important topics pioneered in the early days of HAXPES, we should mention post-collision interaction (PCI). This effect takes place in presence of a slow photoelectron and a fast Auger electron. It is more evident in hard x-ray photoionization, because the core-hole lifetime is shorter and the Auger electron starts earlier and can be more energetic. The physical picture is the following: the slow photoelectron starts to move away from the atom, but then the faster Auger electron passes it. This induces an abrupt change in the potential: the slow photoelectron initially 'feels' the potential of a singly charged ion, but, following emission of an Auger electron, the photoelectron suddenly 'feels' the influence of a doubly charged ion.

The manifestation of PCI is a loss of kinetic energy by the photoelectron, accompanied by a simultaneous gain in kinetic energy by the Auger electron. In the case of photoionization just above the core-level threshold, it is even possible for the photoelectron to be recaptured into an unoccupied Rydberg orbital.

In a paper from 1985, a quantitative measure of this effect was shown, as the photon energy was tuned across the Xe 2 $\mathrm{p}_{3 / 2}$ threshold [23]. 


\subsection{Cascade Auger decay}

The Auger relaxation pathway is very well described in the soft $x$-ray range. The main characteristics there is that the decay takes place usually in one stage (core electron vacancy filled by one valence electron while a second valence electron is emitted). Furthermore, for light elements the radiative decay pattern, i.e. photon emission, is a minority channel and it is usually neglected.

The situation is quite different in the hard x-ray regime. If a deep inner-shell hole is created in a heavy atom, isolated or in a molecule, the subsequent Auger transition can lead to vacancies in states which are still localized (i.e. shallower inner-shell), and in turn can decay via Auger effect in a second step. This process can repeat itself several times, until the final state is a multiply charged atomic or molecular ion with only valence holes, and Auger transitions are not energetically possible anymore. This is usually called cascade Auger, to underline the occurrence of two or more subsequent steps. Furthermore, for heavy atoms the radiative decay channel is not negligible anymore, which means that during the relaxation process interplay of radiative and non-radiative (Auger) decay steps is possible. As an earlier example, argon LMM Auger spectra were measured in coincidence with $\mathrm{K} \alpha$ fluorescent $\mathrm{x}$ rays at several photon energies above the $K$ threshold and on the $1 \mathrm{~s} \rightarrow 4 \mathrm{p}$ resonance [24].

An interesting consequence is that the core-hole-clock definition (see above) holds for each of the cascade steps. This opens the possibility of studying the relaxation dynamics of a series of states created during the decay, which exhibit different lifetime because the vacancies are induced on different core levels, from the deepest to the shallower ones.

\subsection{Double-core-hole studies}

Double-core-hole (DCH) states have recently drawn a lot of interest in the literature. One of the main reasons is that chemical shifts exhibited by DCH states in molecules can be more sensitive to chemical environment effects than the 'historical' chemical shifts described by ESCA, as theoretically demonstrated more than 30 years ago in [25]. Prior to this seminal paper, several experiments were performed on DCH states of neon by heavy ion [26-28] or electron impact [29] sources. However, experimental works on DCH by photoelectron spectroscopy and related techniques have been performed many years later, due to the complexity of the necessary instrumentation. Two categories of experiments have been recently reported in the literature: multi-electron coincidence measurements using synchrotron radiation [30-34], or sequential two-photon absorption experiments with a shortpulse source, therefore conducted at free-electron lasers [35-39]. In the multielectron coincidence measurements, a magnetic bottle spectrometer is used, which allows collecting two photoelectrons and two or three Auger electrons in coincidence, therefore giving a precise picture of the energetics of the double core hole formation. However, this process is governed by electron-electron correlation, implying that this channel is rather weak compared to single-core-hole formation (three to five orders of magnitude less probable) [30-34].

At free-electron lasers, in particular at LCLS, Stanford, California, double-core-hole formation has been achieved by two-photon sequential absorption. While the photon beam is orders of magnitude more intense than the one provided at a synchrotron radiation source, the main requirement of this kind of experiments is to induce the second photon absorption before the Auger decay kicks in, to be able to remove the two core electrons in sequence. This implies that the photon beam should be very short to "beat" the Auger decay. Typical core-hole lifetimes for light elements are in the order of few femtoseconds. Therefore this class of experiments has been conducted at LCLS [35-39], where the pulse duration can be as short as 5 fs. 


\section{Instrumentation}

We present here a brief review of the beam lines and the end stations primarily used during the experimental work which is the subject of this review. We wish to stress the point that the experimental setups located at SOLEIL are operational since few years, and have allowed a major breakthrough in terms of both photon energy resolution and data acquisition, i.e. either measurements of high-resolution photoelectron and Auger spectroscopy (HAXPES), or kinetic energy and position (momentum) of electrons and ions in coincidence (CELIMENE).

\subsection{Beamlines}

\subsubsection{ID 26 (ESRF)}

The RIXS measurements were performed on beam line ID26 at the ESRF in Grenoble, France [40]. This beam line is dedicated to X-ray spectroscopy in the field of applied sciences. It is an insertion device source consisting of three mechanically independent undulators (two 35-mm and one revolving 35/27-mm period). Each mirror of the beamline has three different coatings (Pt, Si and $\mathrm{Pd}$ ) for efficient harmonic rejection. The monochromator is a cryogenically-cooled fixed-exit double-crystal monochromator, with flat $\mathrm{Si}(111)$ pair and flat $\mathrm{Si}(311)$ pair as crystals. The photon energy range is from 2.4 to $27 \mathrm{keV}$. The intrinsic resolution $(\Delta \mathrm{E} / \mathrm{E})$ is $1.4 \times 10^{-4}$ for $\mathrm{Si}(111)$ and $0.3 \times 10^{-4} \mathrm{Si}(311)$. The flux at sample is $>10^{13} \mathrm{ph} / \mathrm{sec}$ using the $\mathrm{Si}(111)$ monochromator, and 5 times less for $\mathrm{Si}(311)$. The beam line provides a high-brilliance $\mathrm{x}$-ray beam, which permits absorption studies even on very dilute samples. It is equipped with a crystal spectrometer to perform $\mathrm{x}$-ray emission spectroscopy. It can also handle different sample environments to perform in situ studies, and can accommodate user experimental stations on an open port (see [40] for more details).

\subsubsection{GALAXIES (SOLEIL)}

The GALAXIES beamline is dedicated to inelastic x-ray scattering (IXS) and hard x-ray photoemission (HAXPES) [41]. These spectroscopic techniques are powerful probes to characterize the electronic properties of matters, either in gas or condensed phase. GALAXIES stands for Great beAmLine for Advanced X-ray Inelastic scattering and Electron Spectroscopies.

The beamline is optimized to operate in the $2.3-12 \mathrm{keV}$ energy range with high resolution and micro beam. The resolution $\Delta \mathrm{E}$ ranges from $100 \mathrm{meV}$ to $1 \mathrm{eV}$ at $8 \mathrm{keV}$. The source is an in-vacuum U20 undulator (20mm period, 98 periods, 0.96 Tesla maximum magnetic field at a minimum gap of $5.5 \mathrm{~mm}$ ) installed in a short straight section of the SOLEIL synchrotron. The main optical components consist of a liquid nitrogen cooled fixed- exit Si(111) double-crystal pre-monochromator (DCM) followed by a 4-bounce high-resolution monochromator (HRM) (which can house a range of different silicon crystals, both symmetric and asymmetric, in order to deliver the required energy resolution, and focusing optics (all manipulated using hexapod positioning systems and operating at grazing incidence angles of 5mrad) toroidal mirrors and a set of $\mathrm{KB}$ mirrors to achieve a micro beam spot at the sample position. A collimating mirror placed upstream of the high-resolution monochromator (M1) ensures maximum through- put. The M1 silicon mirror has palladium and carbon coatings, where $\mathrm{C}$ is used to reject higher order harmonics emanating from the Si(111) DCM when experiments are performed at low x-ray energies. 
The spot size is $20(\mathrm{~V}) \times 80(\mathrm{H}) \mu \mathrm{m}^{2}$ in standard mode, $5(\mathrm{~V}) \times 10(\mathrm{H}) \mu \mathrm{m}^{2}$ in a KirkpatrickBaez (KB) configuration. The flux is $1.5 \times 10^{12}$ photons/s (standard focus), $5 \times 10^{11}$ (microfocus) at $8 \mathrm{keV}$. The beam line is equipped with two permanent end stations, one for RIXS (Resonant Inelastic X-ray Scattering), with a 1-2m radius IXS spectrometer, and one for HAXPES, a high energy / high resolution electron analyzer (30 meV at $12 \mathrm{keV}$ ) (see below).

\subsubsection{LUCIA (SOLEIL)}

LUCIA is one of the two high-energy beam lines at synchrotron SOLEIL where experiments in gas phase can be performed [42].

"LUCIA" stands for Line for Ultimate Characterization by Imaging and Absorption. It works in the so-called "tender" (0.8-8 keV) energy range, which allows reaching the K-edges of elements from $\mathrm{Na}$ to $\mathrm{Fe}$, $\mathrm{L}$ edges from $\mathrm{Ni}$ to $\mathrm{Gd}$, and $\mathrm{M}$ edges of rare earths and actinides.

The source is an undulator of the APPLE-II type with a magnetic structure made of 32 magnets and a period of $54 \mathrm{~mm}$. Linearly polarized light from horizontal to vertical can be obtained, as well as a circularly polarized beam. The source size is $200 \times 20 \mu \mathrm{m}^{2}$.

The optical elements include a spherical mirror for horizontal de-magnification, and two planar mirrors which act as a low pass filter to remove higher harmonics. Their angle of incidence can be varied from $0.4^{\circ}$ to $1.3^{\circ}$. All mirrors are silicon coated with nickel and water cooled. A double crystal monochromator (DCM) is mounted, chosen on the ground of the desired energy range. Its design is based on a double cam which provides a fixed exit geometry and covers a large angular range $\left(5^{\circ}-75^{\circ}\right)$. Up to five different crystals, namely KTP(011), MGM (Multilayer Grating Monochromator), InSb(111), Si(311) and Si(111) fit into the water-cooled holders. The final focusing of the beam is achieved by a KB system. The flux on the sample is in the range $10^{10}-10^{11}$ photons/sec for a beam current of $400 \mathrm{~mA}$ (see [42] for a more detailed description of the beam line).

Its capabilities include $\mathrm{x}$-ray micro-absorption spectroscopy ( $\mu$-XAS) and $\mathrm{x}$-ray microfluorescence ( $\mu$-XRF). Users can also bring their own equipment. In the present framework, a vector correlation chamber (see below) was installed to perform experiments in gas phase.

\subsection{End stations}

\subsubsection{Spectrometer at ESRF}

The experimental apparatus is described in detail in Ref. [43]. It is based on wavelengthdispersive in-vacuum x-ray crystal spectrometer in Johansson geometry. It covers the 2-6 keV energy range. It uses off-Rowland circle target position together with position-sensitive detection of x-rays. It can operate with both focused and broad beam excitation. It has a high resolving power $(>7000)$, which allows achieving energy resolution significantly below the lifetime broadening of characteristic emission lines within the tender $\mathrm{x}$-ray energy range The diffracted photons are detected with a thermoelectrically cooled $\left(-40^{\circ} \mathrm{C}\right) \mathrm{CCD}$ camera with $22.5 \times 22.5 \mu \mathrm{m}^{2}$ pixel size (see [43] for more details). 


\subsubsection{HAXPES (SOLEIL)}

HAXPES is an end station permanently installed on GALAXIES [44]. In Figure 1 we show an overview. The spectrometer which measures the electron kinetic energy is the newly designed EW4000 VG-Scienta hemispherical photoelectron analyzer, optimized for highkinetic-energy electrons (up to $12 \mathrm{keV}$ ). The expected resolution is $35 \mathrm{meV}$ for electron kinetic energy of $10 \mathrm{keV}$. The electron analyzer is mounted on a $\mu$-metal shielded UHV experimental chamber, installed at $90^{\circ}$ from the incident beam and in the horizontal plane of the synchrotron orbit, a configuration suitable for both solid sample and gas-phase experiments. In fact, the chamber is used for both solid-state and gas-phase studies.

For diluted species studies, a gas cell is mounted on a fully motorized 4-axes manipulator located at the bottom of the main chamber.

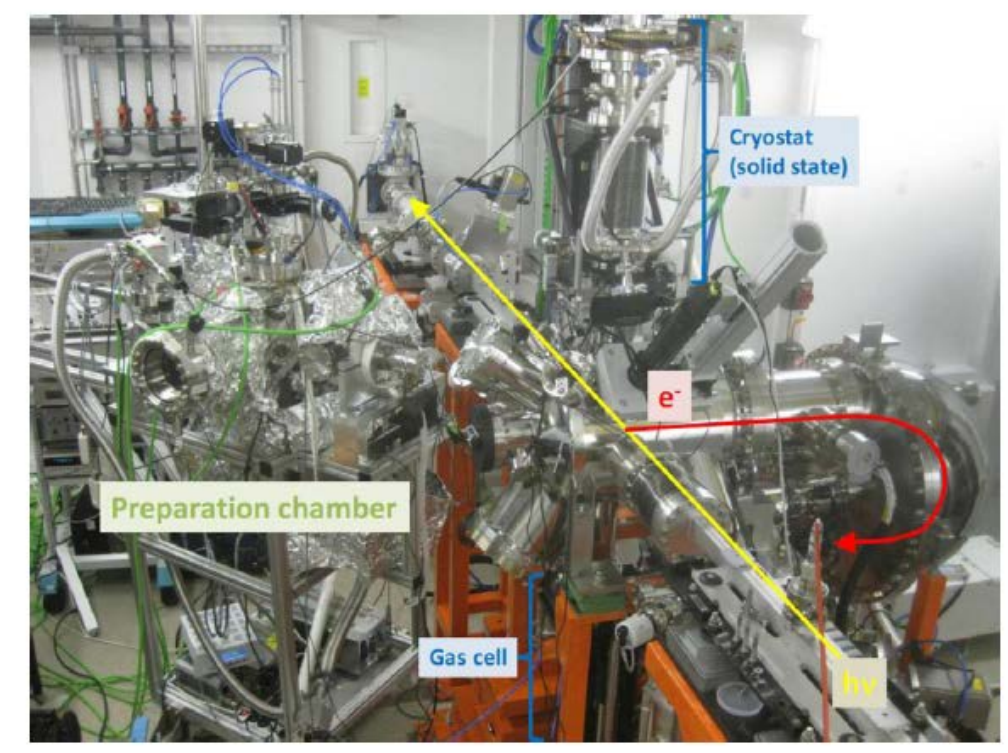

Fig.1. Photo of the HAXPES station at the GALAXIES beamline. The direction of the synchrotron beam is shown in yellow, and the pathway of the collected photoelectrons in red (from [44], reproduced with permission).

\subsubsection{CELIMENE (SOLEIL)}

CELIMENE [45] (Coincidence ELectron Ion Molecule ENErgy) is a momentum spectrometer dedicated to the study of vector correlations, and the measurement of molecular frame photoelectron angular distributions (MFPADs), in the context of dissociative deep core-shell photoionization in the tender x-ray region ( 1 to $10 \mathrm{keV}$ ). It combines time-of-flight and imaging techniques for momentum vector measurements of several charged particles detected in coincidence. It is a double momentum spectrometer of the family of previously developed vector correlation spectrometers. While the original design for those was optimized for low kinetic energies, our apparatus was optimized for particles with kinetic energies of several tens of electron volts.

The experimental setup (Figure 2) is composed of two vacuum chambers: the expansion chamber and the interaction chamber. The expansion chamber houses a molecular beam source. The spectrometer is housed in the interaction chamber. It is composed of two strictly 
identical TOF spectrometers, symmetrically mounted in the interaction chamber with respect to the $(\mathrm{x}, \mathrm{y})$ plane. Electrons and ions are accelerated in opposite direction by a uniform electrostatic field created along the $\mathrm{z}$ axis by two sets of electrostatic lenses polarized with opposite voltages. Each set is made of 7 elements for a total length of $75 \mathrm{~mm}$. A hightransmission mesh (90\%) mounted on the 7th element defines the end of the extraction region and prevents field penetration. The accelerated particles then travel through a $150 \mathrm{~mm}$ long field-free region, or "drift tube". A second mesh is mounted at the end of the tube to prevent field penetration in the field-free region. The particles are collected at the exit of the drift tube by $80 \mathrm{~mm}$ diameter microchannel plates (MCPs) detectors placed $10 \mathrm{~mm}$ behind the exit mesh of the drift tube. The impact position of each particle is measured using commercial delay lines as part of the detector assembly. The impact position coordinates $\mathrm{x}$ and $\mathrm{y}$ measured on the detector and the time of flight $\mathrm{T}$ of the particle through the spectrometer are recorded, and the three components (Px, Py and $\mathrm{Pz}$ ) of the initial momentum vector of each particle can be derived (see [45] for further details).

CELIMENE has been installed on LUCIA, and more recently on an open port on GALAXIES.

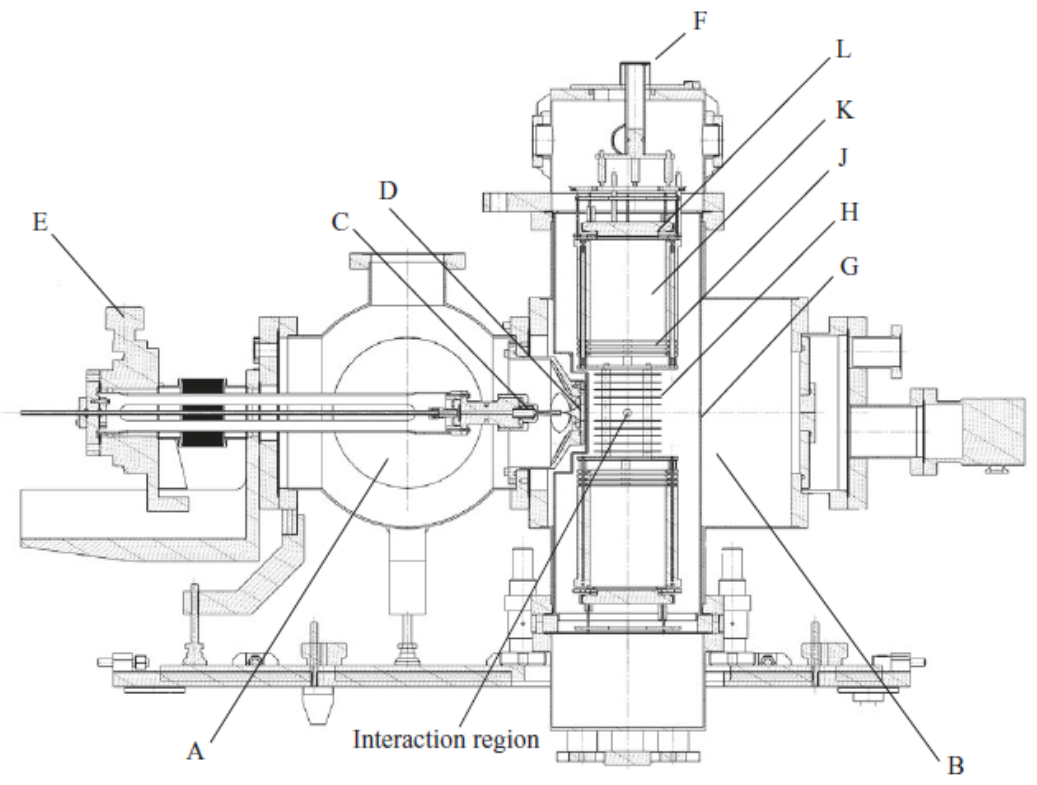

Fig.2. Overview of the experimental setup. A: expansion chamber. B: interaction chamber. C: nozzle. D: skimmer. E: XYZ-manipulator. F: linear manipulator. G: $\mu$-metal shield. H: extraction lens. J: refocusing lens. K: drift tube. L: delay line detector (from [45], reproduced with permission).

\section{$\underline{4 . ~ R e c e n t ~ r e s u l t s ~}$}

In the following paragraphs we will present an overview of the most recent results obtained with the instrumentation described in Section 3. We will start with the ESRF RIXS results (4.1), then continue with the measurements with the HAXPES setup (paragraphs 4.2-4.7), then with experiments performed with both HAXPES and CELIMENE setups $(4.8,4.9)$, and conclude with CELIMENE (4.10). 


\subsection{RIXS: reaching the attosecond timescale}

\subsection{1 $\mathrm{CH}_{3} \mathrm{I}$ and $\mathrm{CH}_{3} \mathrm{Cl}$ : fast nuclear motion revealed}

A pioneering paper [46] has shown the sensitivity of the RIXS spectroscopy to fine details of molecular dynamics upon core photoexcitation on a subfemtosecond timescale.

The showcase system was $\mathrm{CH}_{3} \mathrm{I}$, and the measurements of the RIXS spectra were performed in the hard-x-ray region near the iodine $\mathrm{L}_{2}$ and $\mathrm{L}_{3}$ absorption edges. The main result was that the measured dispersive RIXS spectral features were recognized as a fingerprint of a dissociative molecular state and could be interpreted in terms of ultrashort natural lifetime of 200 attoseconds in the case of the iodine L-shell core-hole. The capability of the RIXS technique to reveal subtle dynamical effects in molecules with sensitivity to nuclear rearrangement on a subfemtosecond time scale was thus demonstrated.

In Figure 3 we show the RIXS spectra presented in the form of a $2 \mathrm{D}$ map, meaning that several RIXS spectra were recorded while changing the photon energy in $0.2 \mathrm{eV}$ steps in the region including the $\mathrm{L}_{3}$ threshold $(\sim 4565 \mathrm{eV})$.

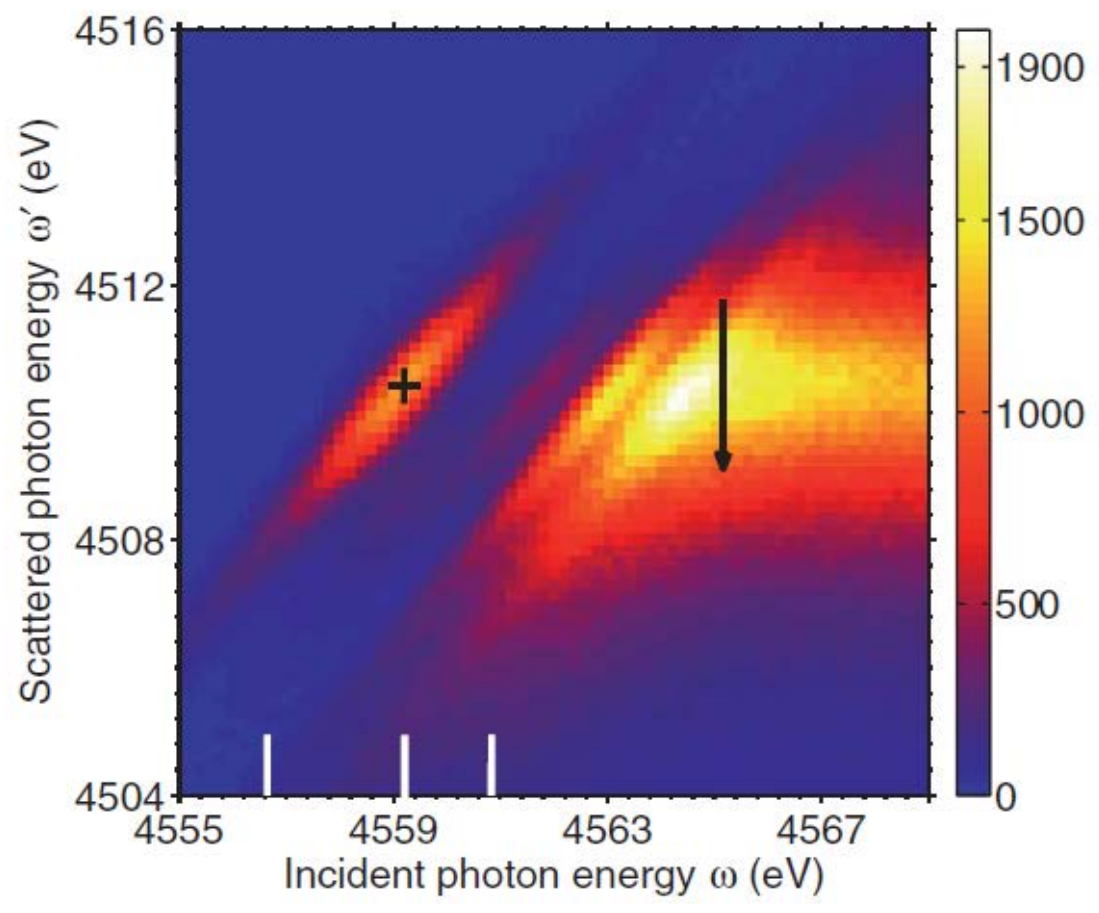

Fig.3. Experimental RIXS spectra in $\mathrm{CH}_{3} \mathrm{I}$ recorded close to $\mathrm{I}_{3}$-edge. The cross around the incident photon energy of $4559 \mathrm{eV}$ shows the maximum of the $\mathrm{L}_{3}-\mathrm{N}_{4,5}$ emission line resulting from a resonant excitation I $2 p_{3 / 2}$ $\rightarrow 15 a_{1}$. The arrow around $4565 \mathrm{eV}$ shows the onset of fluorescence corresponding to an approximate position of $\mathrm{CH}_{3} \mathrm{I}$ ionization threshold (from [46], reproduced with permission).

The cross around the incident photon energy of $4559 \mathrm{eV}$ shows the maximum of the $\mathrm{L}_{3}-\mathrm{N}_{4,5}$ emission line resulting from the resonant excitation $\mathrm{I} 2 \mathrm{p}_{3 / 2} \rightarrow 15 \mathrm{a}_{1}$ (LUMO, lowest unoccupied molecular orbital). The arrow around $4565 \mathrm{eV}$ marks the approximate position of the I $2 \mathrm{p}_{3 / 2}$ ionization threshold. 
More information on the dynamics of the process can be gained by measuring the dispersion and the width of the RIXS features as a function of photon energy.

In particular, the spectral width of a RIXS emission line is a crucial observable, as, along with the line dispersion, it carries information on the evolution of the molecular environment upon the excitation.

In more detail, the cross section for RIXS is given by the Kramers-Heisenberg formula:

$$
\sigma\left(\omega, \omega^{\prime}\right) \propto\left|\sum_{c} \frac{\langle f|D| c\rangle\langle c|D| o\rangle}{\omega-\omega_{c o}+\frac{i \Gamma}{2}}\right|^{2} \delta\left(\omega-\omega^{\prime}-\omega_{f o}\right)
$$

where $\Gamma$ denotes the FWHM (full width at half-maximum) of the natural lifetime broadening of the core-excited state and $D$ is the electric dipole operator, which governs the transitions from the ground state to the intermediate state and from the intermediate state to the final state, $\omega$ and $\omega^{\prime}$ are the incident and emitted photon energies, o, c and $\mathrm{f}$ are the ground, intermediate and final states, $\omega_{\text {co }}$ is the resonant absorption energy, and $\omega_{\mathrm{fo}}$ is the transition energy from the ground state to the final state.

In case the intermediate and final states are dissociative, eq. (1) can be simplified [7]:

$$
\sigma\left(\omega, \omega^{\prime}\right) \propto \frac{\exp \left(-\left[\left(\Omega-\Omega^{\prime} / \Delta\right)\right]^{2} \ln 2\right)}{\Omega^{2}+\Gamma^{2} / 4}
$$

where $\Omega$ and $\Omega$ ' represent the energy detuning from the resonant absorption energy and the resonant emission energy respectively, and $\Delta$ is the width of the Franck-Condon (FC) distribution between the initial and the intermediate state (one FC factor instead of the two FC factors in the numerator of eq.1). This analytical expression can be used to simulate the experimental data, under the main assumption of parallel potential curves of the intermediate and final state (see [46] for details).

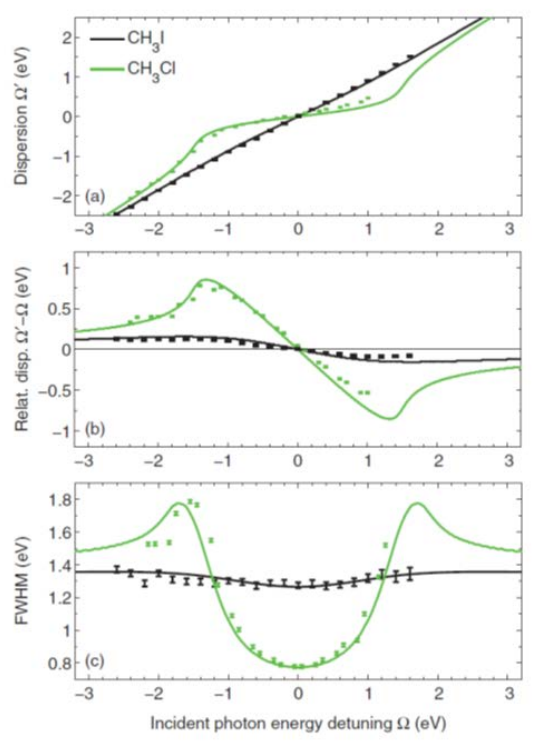

Fig.4. (a) Dispersion, (b) dispersion relative to the linear dispersion, and (c) FWHM of the $\mathrm{I} \mathrm{L}_{3}-\mathrm{N}_{4,5}$ (black) and $\mathrm{Cl} \mathrm{K-L}$ in $\mathrm{CH}_{3} \mathrm{Cl}$ (green) emission lines as a function of the incident photon energy detuning with respect to the resonance energy. Solid lines show simulations using Eq. (3) for RIXS cross section convoluted with the beamline and the spectrometer instrumental function (from [46], reproduced with permission). 
In Figure 4 we show a comparison between the dispersion and the width of RIXS features across the I $2 \mathrm{p}_{3 / 2} \rightarrow 15 \mathrm{a}_{1}$ resonance in $\mathrm{CH}_{3} \mathrm{I}\left(\mathrm{L}_{3}-\mathrm{N}_{4,5}\right.$ emission line, in black) and the $\mathrm{Cl} 1 \mathrm{~s}$ $\rightarrow 8 \mathrm{a}_{1}$ (LUMO) for $\mathrm{CH}_{3} \mathrm{Cl}$ (K-L emission line, in green) measured under the same experimental conditions. The experimental data are compared with simulations obtained by using eq. 2. The rationale behind this direct comparison is that the core-hole lifetime for the I $2 \mathrm{p}_{3 / 2}$ excitation in $\mathrm{CH}_{3} \mathrm{I}$ is much shorter than the one for the $\mathrm{Cl} 1$ s excitation in $\mathrm{CH}_{3} \mathrm{Cl}$, so this is a good test to verify the sensitivity of RIXS to subtle details of the molecular dynamics.

We can observe that the spectral features for both molecules exhibit both a deviation from linear dispersion and a change in width as a function of photon energy. It is also evident that the dispersion law and the width of the spectral features depend critically on the depth of the created core hole: the effects are much stronger for the shallower $\mathrm{Cl}$ 1s hole in $\mathrm{CH}_{3} \mathrm{Cl}$. In particular, in $\mathrm{CH}_{3} \mathrm{Cl}$ dispersion of the peak position is strongly nonlinear around the resonance, whilst $\mathrm{CH}_{3} \mathrm{I}$ shows only a small deviation from the linear dispersion [Fig. 4(top)]. To emphasize this behaviour, in Fig. 4 (middle) the peak position is plotted with respect to linear dispersion; therefore a deviation from zero indicates the presence of nonlinear dispersion. The FWHM of the emission lines (Fig.4, bottom) shows a significant narrowing at the resonance in $\mathrm{CH}_{3} \mathrm{Cl}$, whereas in $\mathrm{CH}_{3} \mathrm{I}$ the effect is hardly visible.

The deviation from linear dispersion can be interpreted as follows: on top of the resonance, a part of the excitation energy is transferred into the nuclear motion. Away from the resonance, the effective scattering time is shortened (see 2.3), the nuclei are effectively frozen, and the linear dispersion of the emission line is recovered. A strong deviation from linear dispersion is present in the $\mathrm{CH}_{3} \mathrm{Cl}$ data (Fig. 4 top and middle), where the $\sim 1 \mathrm{fs}$ natural lifetime of the $\mathrm{Cl} \mathrm{K}$-shell core hole permits considerable nuclear dynamics. In $\mathrm{CH}_{3} \mathrm{I}$ molecule, no significant nuclear motion can occur in the time interval set by the iodine Lshell core-hole lifetime $(0.23 \mathrm{fs})$ : not much energy can be transferred to the nuclei before the photon is emitted. Therefore only a slight deviation of the emission line from the linear dispersion is detected.

Similar considerations can be drawn concerning the line narrowing (see [46] for further details).

We conclude that nuclear dynamics generated in a molecule upon core-hole excitation is clearly reflected in the RIXS spectra, and it depends upon the core-hole lifetime, which sets the time scale of the events. In the $\mathrm{CH}_{3}$ I molecule, the natural lifetime of $\sim 200$ attoseconds of the iodine L-shell core-hole limits the occurrence of nuclear dynamics. However, a slight deviation of the RIXS spectral features around the resonance is still observed, which demonstrates a large sensitivity of RIXS spectroscopy to nuclear dynamics in a molecule, even in the subfemtosecond time range.

We also wish to point put that in a recent paper [47] similar data were obtained with resonant Auger spectroscopy for $\mathrm{CH}_{3} \mathrm{Cl}$, which underlines the complementarity of the two techniques.

\subsection{2: $\mathrm{CS}_{2}$ : accessing the attosecond timescale}

The concept of effective scattering time (see 2.3), is fully exploited in a paper on RIXS experimental and theoretical results on $\mathrm{CS}_{2}$ [48]. In general, an important characteristic to study is the dependence of the spectral profile on the excitation-energy detuning $\Omega$, which allows following the temporal evolution of the electron and/or nuclear wave packets. The natural lifetime of the core hole provides the upper limit of the scattering time under resonant conditions, i.e. for $\Omega=0$ (see eq.1).

The main results are contained in Figure 5, which shows the experimental (red) and the calculated (black) S K $\beta$ RIXS spectra as a function of photon energy detuning from the resonant value (2470.4 eV, corresponding to the $\mathrm{S} 1 \mathrm{~s} \rightarrow 3 \pi_{\mathrm{u}} \mathrm{LUMO}$ transition). A significant 
variation of the spectral shape is evident while going towards both positive and negative detunings.

In particular, for positive detuning values, when the photon energy goes above the LUMO excitation energy, contributions from the excitation of higher MOs are visible. At the resonance energy, the emission bands labelled as A, B, and C in Figure 5, can be assigned to transitions to the final states $\left|2 \pi_{\mathrm{g}}{ }^{-1} 3 \pi_{\mathrm{u}}{ }^{1}>,\right| 2 \pi_{\mathrm{u}}{ }^{-1} 3 \pi_{\mathrm{u}}{ }^{1}>$, and $\mid 5 \sigma_{\mathrm{u}}{ }^{-1} 3 \pi_{\mathrm{u}}{ }^{1}>$. Interestingly, band $\mathrm{A}$, prominent in all spectra from the top of the resonance to negative detunings, corresponds to a symmetry-forbidden transition from the gerade electronic ground state $\Sigma_{\mathrm{g}}{ }^{+}$to the ungerade final state.

Furthermore, the evolution of the RIXS profile towards large negative detuning values $(\Omega=-$ $10 \mathrm{eV}$ ) shows a relevant relative enhancement of the $\mathrm{B}$ and $\mathrm{C}$ emission bands and the appearance of additional transitions at the low-energy tail of the RIXS spectrum.

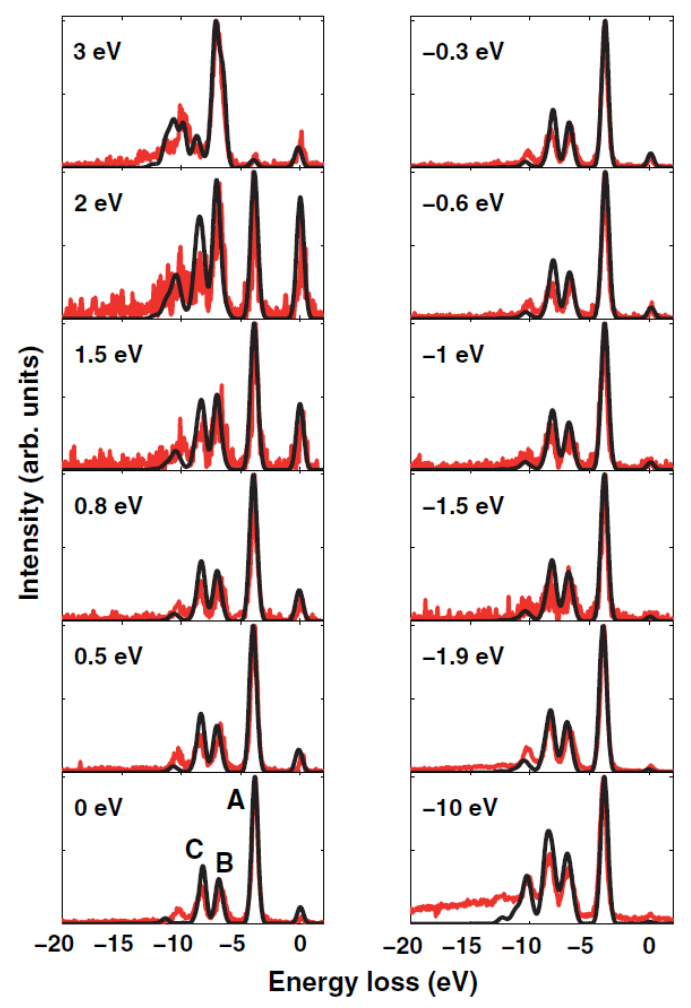

Fig.5. Experimental (red line) and calculated (black line) S K $\beta$ RIXS spectra of the $\mathrm{CS}_{2}$ molecule for different values of the incident photon-energy detuning $\Omega$ from the $1 \mathrm{~s} \rightarrow 3 \pi_{\mathrm{u}}$ LUMO resonance (from [48], reproduced with permission).

We can rationalize these findings as follows: the appearance of the dipole-forbidden transition is related to a mechanism which is typical in the hard x-ray regime, and namely the quenching of the Young's double-slit (YDS) interference, simply because the $x$-ray wavelength is shorter than the internuclear distance. The equivalent of the YDS interference in molecular photoexcitation is the presence of two chemically equivalent atoms, which act as centres of coherent electron or photon emission, and therefore an interference pattern conceptually similar to the well-known YDS experiment can be measured. While this equivalence has been amply studied in the soft x-ray regime (see e.g. [49] and references therein), in $\mathrm{CS}_{2}$ deep-core excitation the two $\mathrm{S}$ centres do not act as equivalent slits (in the YDS model), due to the wavelength of the emission being shorter than the S-S distance. Therefore the core hole can be considered localized on one of the $\mathrm{S}$ atoms, and as a 
consequence a symmetry breaking takes place which leads to the appearance of the forbidden band in the RIXS profile (see [48] and references therein for more details).

The other relevant feature of the RIXS spectra in the negative detuning region reflects the temporal evolution of the multielectron wave packet in the presence of a deep S 1s core hole, and reveals the underlying electron dynamics, which originates from interference of coherently excited multielectron core-hole states.

More in detail, when the excitation energy is tuned significantly below the LUMO resonance, all unoccupied MOs can be coherently excited, and then interference between all the intermediate multielectron core-excited states can occur.

A coherent superposition of excited electronic states can be created in the hard x-ray regime because of the large lifetime broadenings, which enhances the overlap of the deep coreexcited states. In this regime, the spectral shape of RIXS is determined by the interplay of the scattering time and the characteristic time of the electron wave-packet evolution. As $\Omega$ increases, the effective scattering becomes shorter than the characteristic time of the evolution of the electron wave packet, and the scattering amplitude gradually approaches the limit of fast scattering, in which the molecule experiences an instantaneous quadrupole transition from the ground to the final state. It is demonstrated that the convergence of the RIXS spectrum to the limit of fast scattering occurs for detuning $\Omega \approx-100 \mathrm{eV}$, where the scattering time falls below 10 attoseconds (see [48] for further details). The conclusion is that by performing RIXS measurements in the hard x-ray domain the attosecond timescale can be reached even with a continuous source like synchrotron radiation.

\subsection{Double-core-hole studies}

In section 2.6 we have illustrated the existing literature concerning double-core-hole studies. While both types of experiments (multielectron coincidence studies with synchrotron radiation or two-photon sequential ionization with short light pulses) are very demanding in terms of experimental conditions, more recently we found a third simpler way to investigate double core holes. In particular, DCH states may be studied relatively easily via standard single-channel photoelectron spectroscopy using synchrotron radiation [50-58]. In the HAXPES apparatus (see 3.2.2) we can measure one ejected photoelectron at a time, therefore we can measure spectra of what can be labelled a super shake-up (satellite) state, and namely 1s photoionization accompanied by shake-up of a core ns or np electron to some unoccupied orbitals.

The first prototypical system investigated is argon [50].

Assuming that monopole satellite transitions dominate in argon, there are two possible states corresponding to double core-hole formation:

1) $\operatorname{Ar} 1 s^{-1} 2 p^{-1} n p\left({ }^{2} S_{1 / 2}\right) \varepsilon p$

2) $\operatorname{Ar} 1 \mathrm{~s}^{-1} 2 \mathrm{p}^{-1} \mathrm{~ns}\left({ }^{2} \mathrm{P}_{1 / 2,3 / 2}\right) \varepsilon \mathrm{s}, \varepsilon \mathrm{d}$

The states of type (1) can be considered as Ar 1s ionization accompanied by shakeup of a $2 p$ core electron into an unoccupied np Rydberg orbital. The states of type 2 are due to Ar $2 p$ ionization accompanied by shakeup of a 1 s electron into an unoccupied ns Rydberg orbital.

In Figure 6 we show the photoelectron spectrum of $\mathrm{Ar} 1 \mathrm{~s}^{-1} 2 \mathrm{p}^{-1} \mathrm{nl} \mathrm{DCH}$ shake-up states (experimental spectrum together with a fit).

Several Rydberg series converging to the $1 \mathrm{~s}^{-1} 2 \mathrm{p}^{-1}$ thresholds are visible in the spectrum; therefore the energies for the DCH thresholds can be derived. 
In addition, by a careful fitting procedure the lifetime broadening of the final states was determined. The obtained value of $675 \mathrm{meV}$ is close to the value of a single Ar 1s hole (655 $\mathrm{meV}$ ) but not with the sum of a single Ar 1s hole and a single 2p hole (115 meV). This decrease of the Ar 1s core-hole lifetime broadening is attributed to a decrease of the Auger rates caused by the vacancy in the $2 p$ shell.

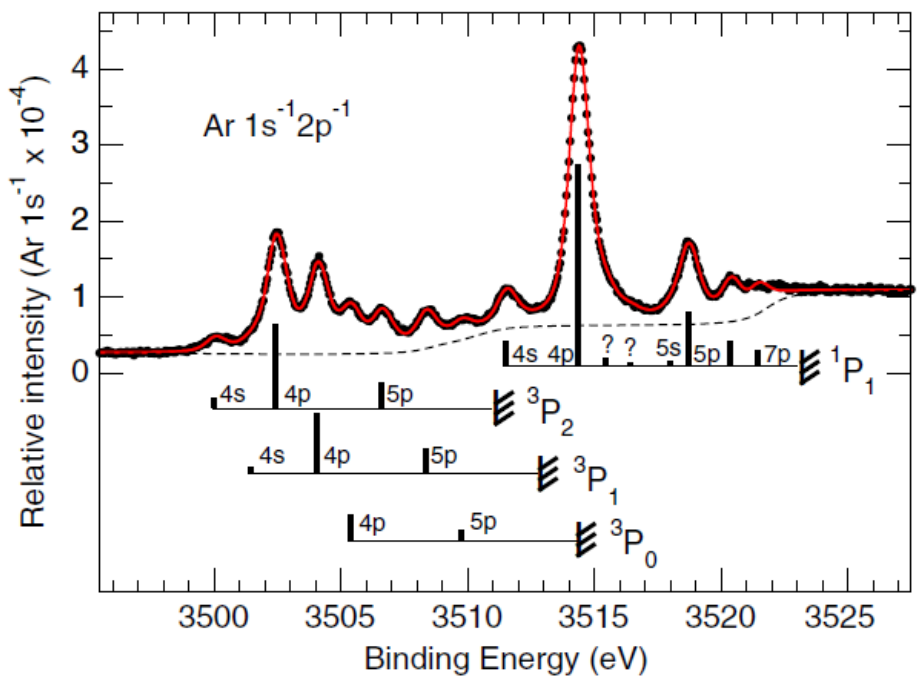

Fig.6. $1 \mathrm{~s}^{-1} 2 \mathrm{p}^{-1} \mathrm{DCH}$ shake-up photoelectron spectrum of argon. The experimental points are shown together with a solid line which is the result of a fit. The bar diagrams indicate positions and intensities of Rydberg series converging to the four different double ionization thresholds (from [50], reproduced with permission).

While in argon double core holes with one vacancy in the K-shell and one in the L-shell were studied, the following step was to investigate neon, where it has been possible to create states with a double K-shell vacancy [51] and also to study the Auger decay of such states [52].

In Figure 7 we show the photoelectron spectrum of the super shakeups in Ne, i.e. state with two holes in the K-shell and one excited electron in a Rydberg state. Both the ns and the np Rydberg series are clearly observed. According to the standard shake up model, direct and conjugate shake up states can be distinguished, i.e. states with a dipolar photoelectron emission and a monopole excitation (direct process, in the present case the $1 \mathrm{~s}^{-2} \mathrm{~ns}$ Rydberg series) or states with a dipole excitation and a photoelectron shake off emission (conjugate process, the $1 \mathrm{~s}^{-2} \mathrm{np}$ Rydberg series).

Experimental values for the core-hole lifetime broadening were also obtained. It is interesting to compare the results with the argon case. The obtained value for the $1 \mathrm{~s}^{-2} \mathrm{Ryd}$ states in neon is $701 \mathrm{meV}$, which is about 2.9 times the broadening for a single 1s hole. This effect is explained by the contraction of the $n=2$ orbitals, involved in the Auger decay (see [51] for details ion the model used). The $\mathrm{n}=2$ orbitals shrink more for DCH states than for SCH ones. Therefore the overlap with the final states, and consequently the Auger decay rate, increases, causing the shortening of the core-hole lifetime. 


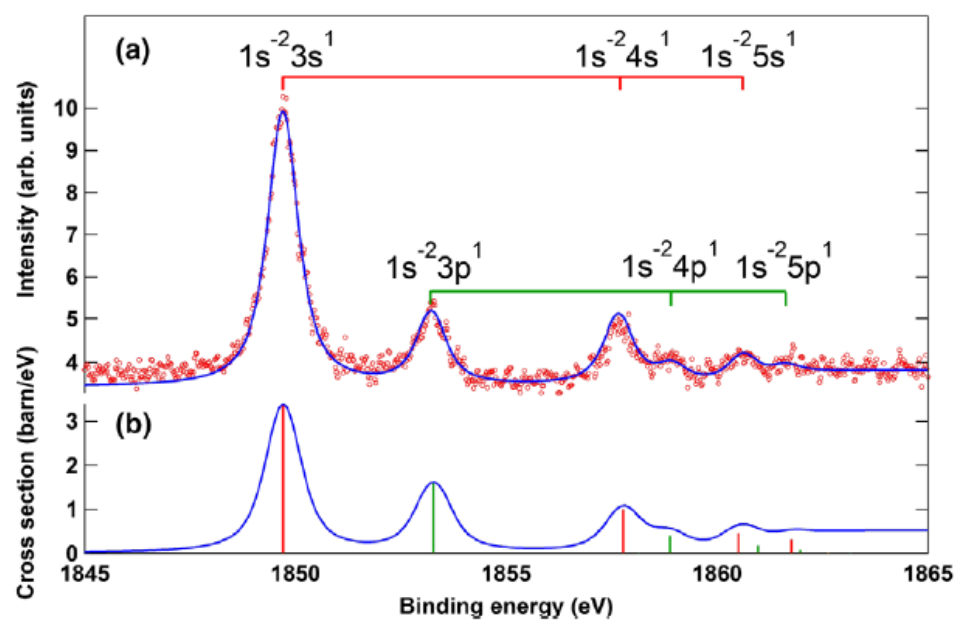

Fig.7. (a) $\mathrm{K}^{-2} \mathrm{~V}$ photoelectron spectrum of Ne measured at $2.3 \mathrm{keV}$. The red dots are the experimental points and the solid blue line is the result of the fit. The photoelectron peaks are identified and two different Rydberg series are observed, corresponding to direct $\left(1 \mathrm{~s}^{-2} \mathrm{~ns}^{1}\right)$ and conjugate $\left(1 \mathrm{~s}^{-2} \mathrm{np}^{1}\right)$ contributions. (b) Calculated photoelectron spectrum (from [51], reproduced with permission).

To cast a deeper look at this process, the Auger decay was studied, in particular the energy region of the so-called hypersatellites, i.e. Auger final states reached by the decay of doublecore-hole states [52]. Such Auger electrons are more energetic than the diagram Auger emission following the decay of a single core hole; therefore those can be easily separated. However, their detection is rather challenging, since they sit on a high background which is the tail of the single-hole Auger decay spectrum. The experimental Auger hypersatellite intensity has to be multiplied by a factor of 2,000 to make the structures clearly visible.

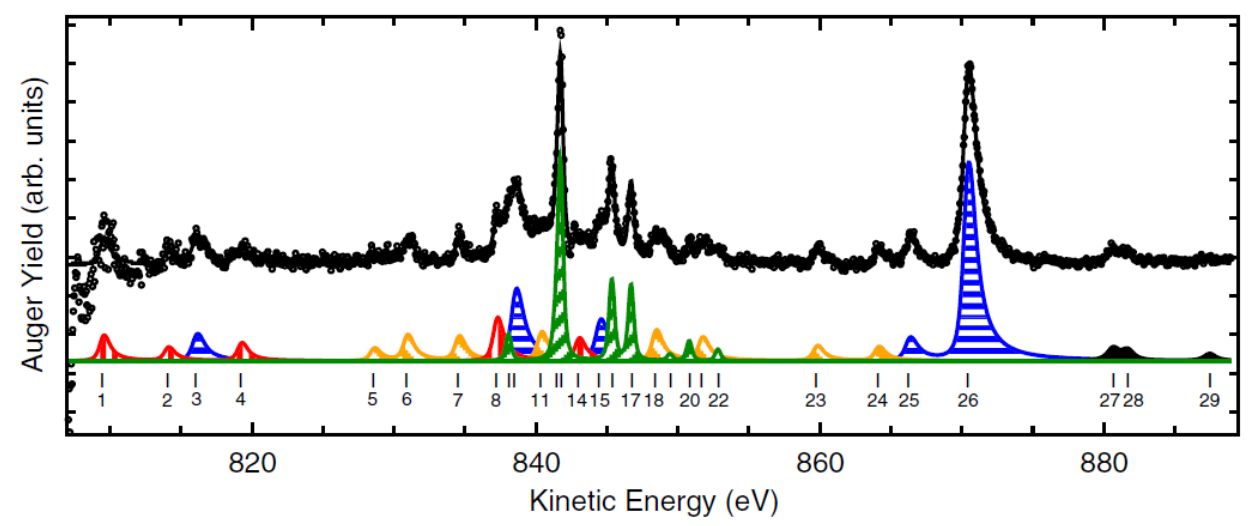

Fig.8. Hypersatellite Auger spectrum of neon (see text for a detailed assignment) (from [52], reproduced with permission).

In Figure 8 we show the Auger hypersatellite spectrum. The black curve is the experimental spectrum after background subtraction, and the peaks in colour are the results of a fit analysis (see [52] for details). 
Three different categories of spectral features are noticed: narrower ones (in green), wider low-intensity ones (in yellow), and finally wider blue ones with a pronounced asymmetric high-kinetic-energy tail.

The narrower peaks correspond to the decay of core-hole state with a longer lifetime; therefore they are assigned as satellites of the Ne $1 \mathrm{~s}^{-1}$ Auger decay.

The other structures have the same larger lifetime broadening, and then the first step in their attribution is to relate them to the decay of states with a double core hole. An interesting point is that some of them (in yellow) do not show a clear tail, at variance with the other ones (in blue).

A ready general explanation for such a tail in studies of Auger decay is post-collision interaction (PCI): when the fast Auger electron overtakes the slow photoelectron, the sudden change in Coulomb potential causes an acceleration for the Auger electrons which is reflected in the high-energy tail.

To observe PCI, in general it is necessary to be close to an ionization threshold, so that slow photoelectrons are emitted. In the present case this condition is not fulfilled. However, looking in more detail to the dynamics of photoelectron emission, it is known that when two photoelectrons are emitted at the same time they share the energy, and typically the energysharing curve is U-shaped, meaning that there is one slow and one fast photoelectron. Therefore in the present case the Auger peaks with the asymmetric tail can be attributed to the decay of states of the type $\mathrm{Ne} 1 \mathrm{~s}^{-2}$, with two core holes. We cannot measure them in the photoelectron spectrum, because it would be necessary to collect the two photoelectrons in coincidence and we perform single-channel measurements. Nevertheless, we can detect their Auger decay, which takes place in two sequential steps and therefore only one electron is emitted at a time.

As for the broad peaks in yellow, they correspond to the first step of the decay of the super shake ups $\mathrm{Ne} 1 \mathrm{~s}^{-2} \mathrm{Ryd}$ (a corresponding photoelectron spectrum is shown in Figure 7). In the latter case, no slow photoelectrons are present, and therefore no clear PCI tail is detected. The experimental results were very well reproduced by a subsequent theoretical paper [53].

The next step was to extend this research line to molecules, and investigate which kind of new information can be gathered.

The first systems studied are two sulphur-containing molecules, $\mathrm{CS}_{2}$ and $\mathrm{SF}_{6}$ [54]. In both cases, double-core-hole states were studied at two thresholds, the S 1s and the C 1s - F 1s respectively. The binding energies of these states are calculated using a Hartree-Fock and a post Hartree Fock (Møller Plesset at second order level of perturbation, MP2) code from the GAMESS package. In addition, Density Functional Theory (DFT) calculations are performed for core-hole formation on $\mathrm{C}$ and $\mathrm{F}$ atoms.

In Figure 9 (bottom) we show the measured photoelectron spectrum for $\mathrm{CS}_{2}$ in the energy region around the $\mathrm{C} 1 \mathrm{~s}^{-2}$ threshold. The calculated spectrum is shown on top. Red and green curves correspond to direct and conjugated shakeups respectively. The special asset of such kind of spectroscopy is evident also in a molecular case: compared to a "normal" absorption spectrum, we can observe more spectral structures, because in the present case peaks corresponding to dipole-forbidden transitions are clearly identified. Analogous data are reported for the $S$ 1s threshold (not shown). In the $\mathrm{SF}_{6}$ case, in Figures 10 and 11 we show the spectra around the $\mathrm{S} 1 \mathrm{~s}$ and the $\mathrm{F} 1 \mathrm{~s}$ thresholds respectively. The experimental spectra are reported on the bottom and the theoretical curves on the top for both figures. 


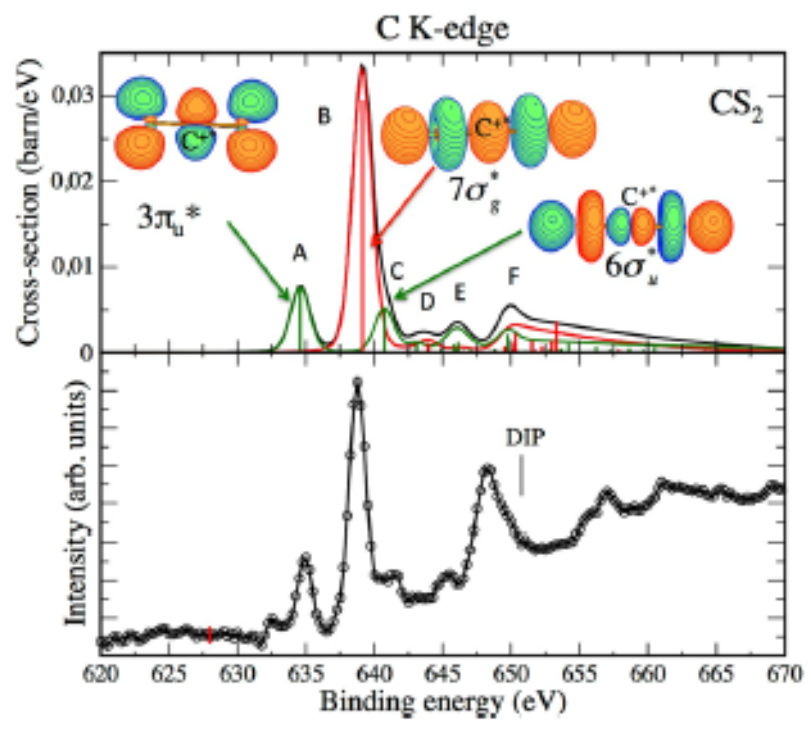

Fig.9. Experimental (lower panel) and theoretical (upper panel) $\mathrm{C} K^{-2} \mathrm{~V}$ spectrum of $\mathrm{CS}_{2}$. The main peaks are illustrated by electronic iso-density plots. Red lines: direct contributions; green lines: conjugate contributions; black curve: incoherent sum of direct and conjugate contributions (from [54], reproduced with permission).

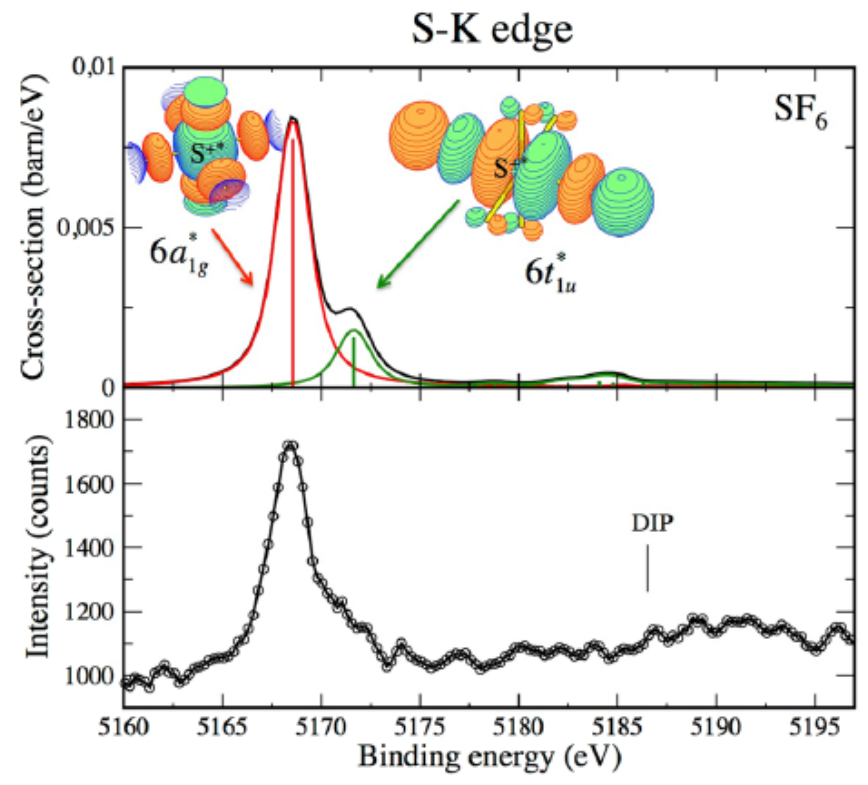

Fig.10. Experimental (lower panel) and theoretical (upper panel) sulphur $K^{-2} \mathrm{~V}$ spectra of $\mathrm{SF}_{6}$. Red lines reflect the direct contributions and green lines reflect conjugate contributions. The black curve represents an incoherent sum of the direct and conjugate contributions (from [54], reproduced with permission). 


\section{F-K edge}

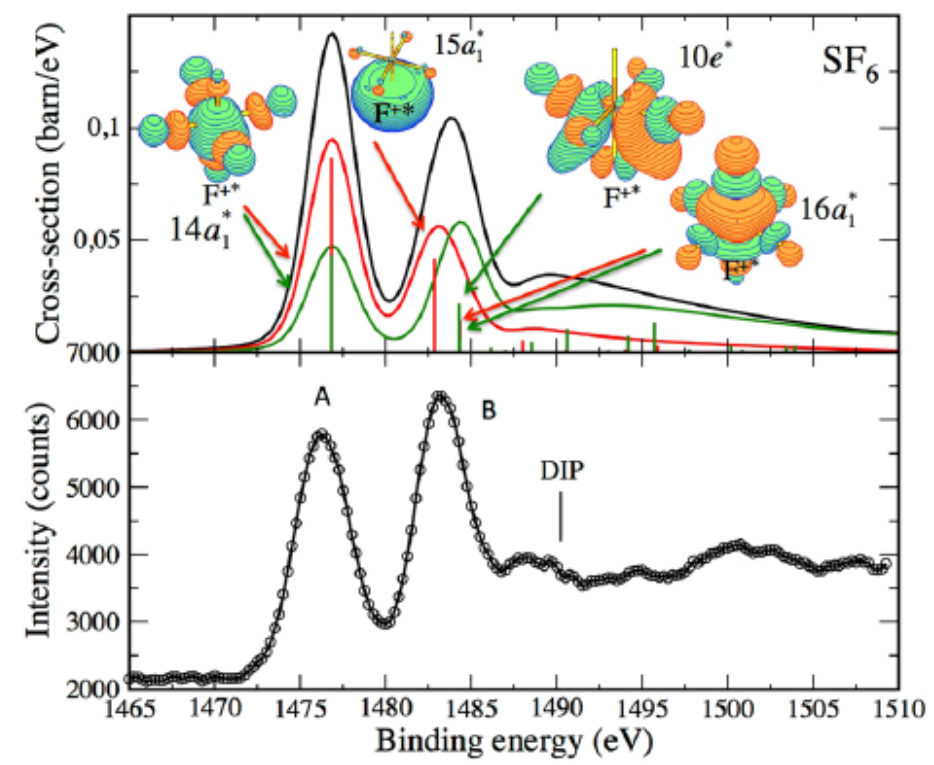

Fig.11. Experimental (lower panel) and theoretical (upper panel) $\mathrm{F} \mathrm{K}^{-2} \mathrm{~V}$ spectra of $\mathrm{SF}_{6}$ (from [54], reproduced with permission).

Apart from the above mentioned finding that both dipole-allowed and dipole-forbidden transitions can be identified, another important ability of this kind of spectroscopy is the following: some resonance well-known to appear in a ionization continuum, namely the shape resonances, appear in the $K^{-2} \mathrm{~V}$ spectra, but below the ionization threshold and not above. Examples are the weak structure at about $5185 \mathrm{eV}$ binding energy in Figure 10, and the peak at $1490 \mathrm{eV}$ in Figure 11. The shape resonances are known to depend upon subtle details of the molecular potential. Therefore the relevant change in molecular potential experienced by the outgoing photoelectron from single- to double-core-hole formation seems to be responsible for the shape resonances to be pushed below an ionization threshold. This can be considered a general effect in this kind of spectroscopy.

Another molecule studied is $\mathrm{HCl}$, isoelectronic with argon [55].

In Figure 12 we show a spectrum for $\mathrm{HCl}$ taken in the energy region of states of the type $\mathrm{Cl}$ $1 s^{-1} 2 s(2 p)^{-1} V$, analogous to the Ar case. Again, Rydberg series converging to the various double ionization thresholds can be identified, and the values for the threshold energy determined quite accurately. 


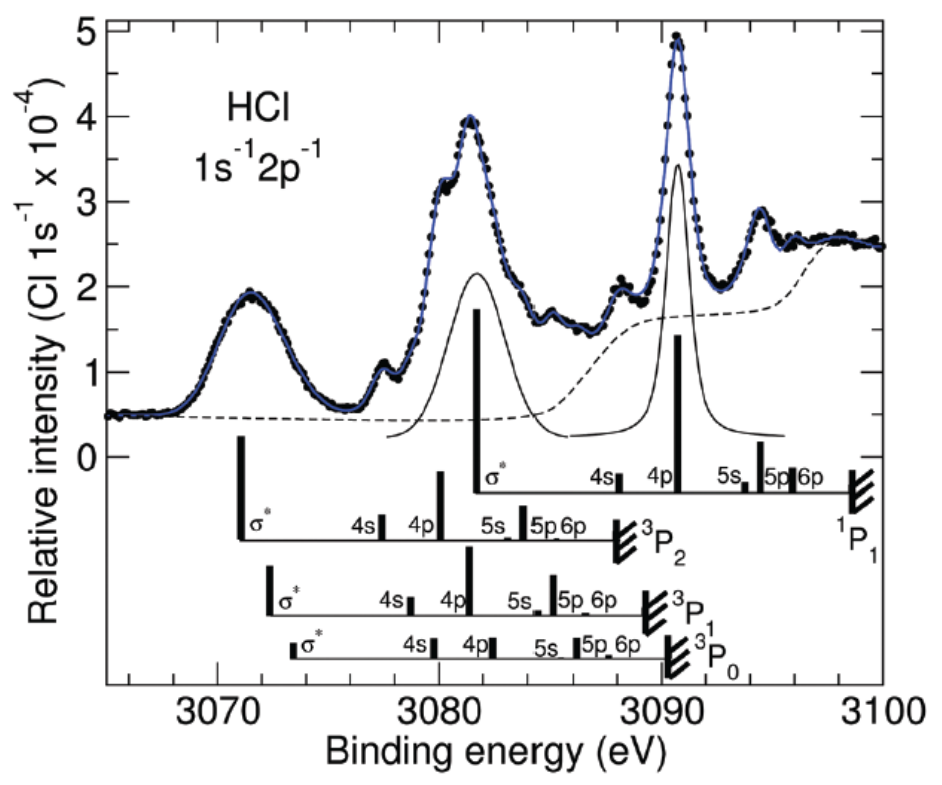

Fig.12. Photoelectron spectrum of $\mathrm{HCl}$ in the region of the $1 \mathrm{~s}^{-1} 2 \mathrm{p}^{-1}$ excitations. The vertical-bar diagrams in the lower part indicate the energy positions and intensities for the various Rydberg series converging to four double ionization thresholds (from [55], reproduced with permission).

raper

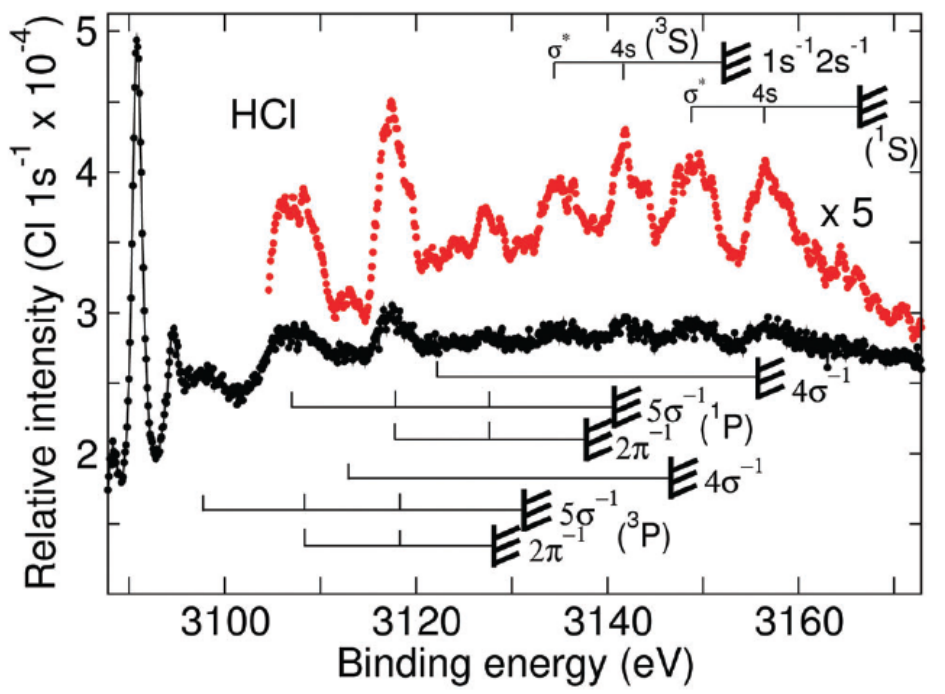

Fig.13. Photoelectron spectrum in the energy region between $3088 \mathrm{eV}$ and $3173 \mathrm{eV}$. The peaks in the region from 3088-3148 eV are assigned as corresponding to double shake-up states, i.e. states with two core holes, one valence hole and two excited electrons (e.g. $1 \mathrm{~s}^{-1} 2 \mathrm{p}^{-1}\left({ }^{1,3} \mathrm{P}\right) 5 \mathrm{~s}^{-1} \mathrm{~s}^{*^{2}}$ ). Triple ionization thresholds are marked in the lower part. Such features overlap with the $1 \mathrm{~s}^{-1} 2 \mathrm{~s}^{-1} \mathrm{~V}$ shake regions in the range from $3137-3173 \mathrm{eV}$. In the upper part the thresholds for states of the type $1 \mathrm{~s}^{-1} 2 \mathrm{~s}^{-1}$ are also marked (see text for more details) (from [55], reproduced with permission).

In addition, a spectral region corresponding to more complicated processes such as double shake up has been analysed in detail, and threshold energies even for triple ionization events have been assigned, as shown in Figure 13. Double shake ups are defined as states with two core holes, one valence hole and two excited electrons. In particular, the two most intense 
peaks at 3098 and $3107 \mathrm{eV}$ are assigned to states of the type $1 \mathrm{~s}^{-1} 2 \mathrm{p}^{-1}\left({ }^{1,3} \mathrm{P}\right) 5 \mathrm{~s}^{-1} \mathrm{~s}^{* 2}$. The thresholds marked in the lower part of Figure 13 correspond to triple ionization ones, such as e.g. $1 \mathrm{~s}^{-1} 2 \mathrm{p}^{-1}\left({ }^{1,3} \mathrm{P}\right) 5 \mathrm{~s}^{-1}$. In the upper part of Figure 13 some states are assigned to $1 \mathrm{~s}^{-1} 2 \mathrm{~s}^{-1} \mathrm{~V}$ shakeups overlapping with the double shakeup series. The $1 \mathrm{~s}^{-1} 2 \mathrm{~s}^{-1}$ ionization thresholds are also marked in the upper part of the figure.

A recent system investigated is $\mathrm{CH}_{3} \mathrm{CN}$, acetonitrile [56]. The interest in this molecule mainly resides in the fact that there are two chemically inequivalent carbon atoms, but their chemical shift is too small to be revealed even with high-resolution core photoemission.

Photoelectron spectra were measured around the $\mathrm{N}$ 1s double-core-hole threshold (not shown) and around the $\mathrm{C}$ 1s one, shown in Figure 14.

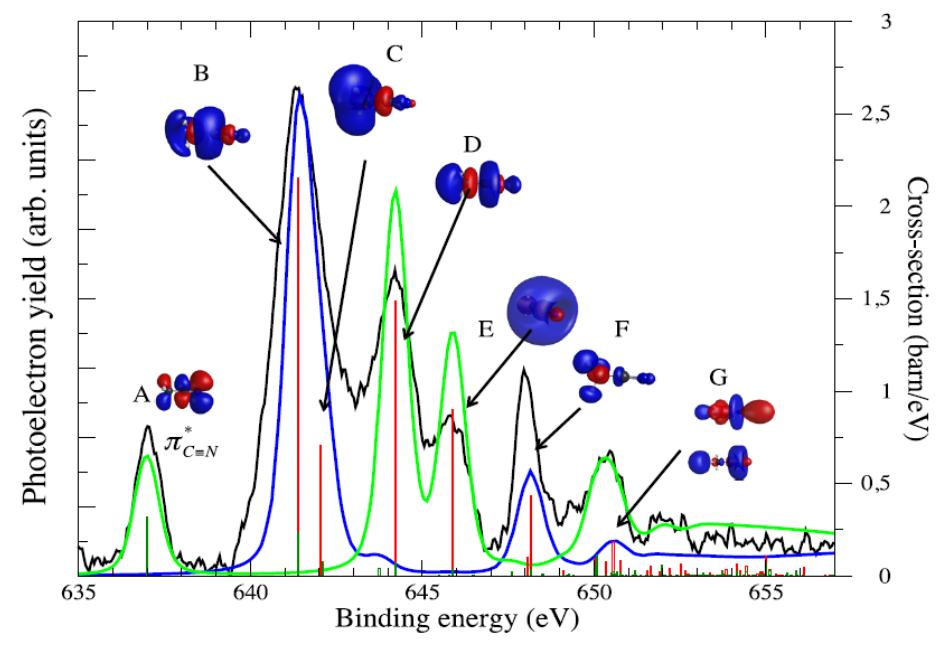

Fig.14. Experimental satellite photoelectron spectrum, showing the formation of $\mathrm{C} 1 \mathrm{~s}^{-2} \mathrm{~V}$ DCH states, recorded at $h v=2300 \mathrm{eV}$ (black line), along with the theoretical spectrum showing the formation of $\mathrm{C}^{*} \mathrm{~N} 1 \mathrm{~s}^{-2} \mathrm{~V} \mathrm{DCH}$ states (light green line) and $\mathrm{C}^{*} \mathrm{H}_{3} 1 \mathrm{~s}^{-2} \mathrm{~V}$ DCH states (blue line). The direct and conjugate character of each transition is also depicted in red and green, respectively. The MOs of the final states are also shown (from [56], reproduced with permission).

In the theoretical spectrum reported in the figure, blue and green lines are the colour codes for $\mathrm{C} 1 \mathrm{~s}^{-2} \mathrm{~V}$ states mainly localized on the carbon of the methyl group (blue) or the carbon of the CN group (green). It is evident that with this technique a large separation can be measured between super-shakeups with main contribution of one of the two inequivalent carbons, even if the chemical shift in the "normal" C 1s photoelectron spectrum is very small.

The most recent molecule investigated is water [57]. A combined experimental and theoretical investigation is reported of the Auger emission processes following double core ionization and excitation of gas-phase water molecules with hard-x-ray synchrotron radiation in the photon energy region above the $\mathrm{O}$ K-edge double ionization. The main result of this work is an indication of ultrafast proton motion occurring within the very short lifetime of the DCH states in water (about $1.5 \mathrm{fs}$ ). In addition, a closer look over the nuclear dynamics following DCH formation was obtained, and symmetric and antisymmetric dissociation patterns connected to specific DCH states could be identified.

In Figure 15 we show the hypersatellite Auger spectra, i.e. the Auger electron emission following the double-core-hole formation. The experimental spectra are compared with theoretical ones taking into account the nuclear dynamics (top panel) or at fixed geometry (bottom panel). The agreement is much better with the top panel, in particular for the spectral 
region above $560 \mathrm{eV}$, where there is a long tail attributed to the effect of nuclear motion (see [57] and references therein for more details).
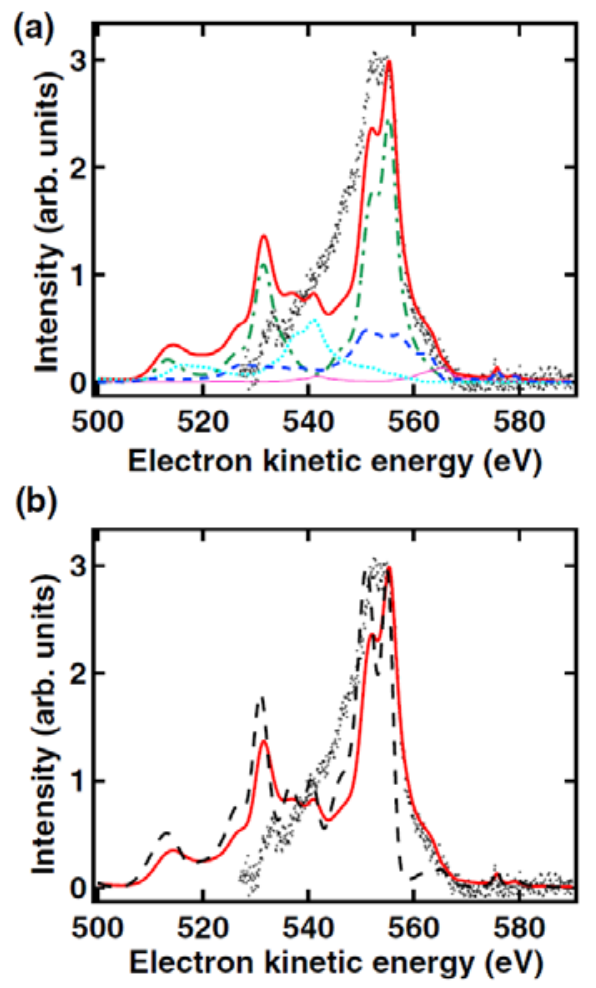

Fig.15. (a) Comparison of an experimental hypersatellite Auger spectrum of water measured at $2.3 \mathrm{keV}$ (black circles) with the Auger spectra calculated including the nuclear dynamics. The total Auger spectrum (thick solid red line) is composed of various hypersatellite contributions. (b) Comparison of the experimental HS Auger spectra (black circles) to the calculation with fixed molecular geometry (dashed black) and with the nuclear dynamics included (solid red) (from [57], reproduced with permission).

\begin{tabular}{lccc}
\hline \hline Configuration & $\begin{array}{c}\text { Electronic } \\
\text { state }\end{array}$ & $\begin{array}{c}\text { Relative } \\
\text { weight (A) }\end{array}$ & $\begin{array}{c}\text { Relative } \\
\text { weight (B) }\end{array}$ \\
\hline$K^{-2}$ & $K^{-2}$ & 0.56 & 0.48 \\
$K^{-2} V$ & $K^{-2} 4 a_{1}$ & 0.02 & 0.05 \\
$K^{-2} V$ & $K^{-2} 2 b_{2}$ & 0.02 & 0.05 \\
$K^{-2} L^{-1} V$ & $K^{-2} 3 a_{1}^{-1} 4 a_{1}$ & 0.06 & 0.10 \\
$K^{-2} L^{-1} V$ & $K^{-2} 2 a_{1}^{-1} 4 a_{1}$ & 0.09 & 0.10 \\
$K^{-2} L^{-1} V$ & $K^{-2} 1 b_{2}^{-1} 2 b_{2}$ & 0.07 & 0.11 \\
$K^{-2} L^{-1}$ & $K^{-2} 3 a_{1}^{-1}$ & 0.03 & 0.02 \\
$K^{-2} L^{-1}$ & $K^{-2} 1 b_{2}^{-1}$ & 0.07 & 0.08 \\
$K^{-2} L^{-1}$ & $K^{-2} 2 a_{1}^{-1}$ & 0.09 & 0.02 \\
\hline \hline
\end{tabular}

Table 1. Relative weights of different intermediate electronic states contributing to the HS Auger spectrum. Column A shows weights that are according to the distribution found in neon; Column B shows weights with a reduced contribution for the main satellite $K^{-2}$ and a stronger contribution for the $K^{-2}{ }^{-1} V$ satellites (see [57] for details). 
The different colours of the theoretical curves refer to the Auger decay of different DCH states (see [57] for a detailed assignment). A list of the various decay paths including their relative weight is reported in Table 1.

To explain the tail present on the high-energy side of the main peak, several possible factors have been taken into account: the Lorentzian lineshape, the relative weight of the various decay channels, and the PCI (post-collision interaction). We demonstrate that none of these effects can explain the long tail.

The real explanation relies on nuclear dynamics. Our calculations for the $\mathrm{O} \mathrm{K}^{-2}$ state predict symmetric dissociation into $\mathrm{H}^{+}+\mathrm{O}+\mathrm{H}^{+}$within several femtoseconds. The dissociation dynamics in the $K^{-2} 4 a_{1}$ state is even faster, due to the antibonding character of the $4 a_{1}$ orbital. Our calculations show that for such state there is a strong contribution along the antisymmetric stretching mode, resulting in one short and one long bond distance. Propagation of the nuclear wave packet along the antisymmetric stretching mode leads to considerable additional broadening of the $K^{-2} 4 a_{1}$ component of the Auger hypersatellite spectrum, which explains the high-energy tail.

In Figure 16 we show the evolution of the average O-H bond distance for different electronic states, showing the interplay between their dissociative character and the fast elongation of the $\mathrm{O}-\mathrm{H}$ bond. In other words, we "catch" in the Auger spectra the signature of a ultrafast proton motion.

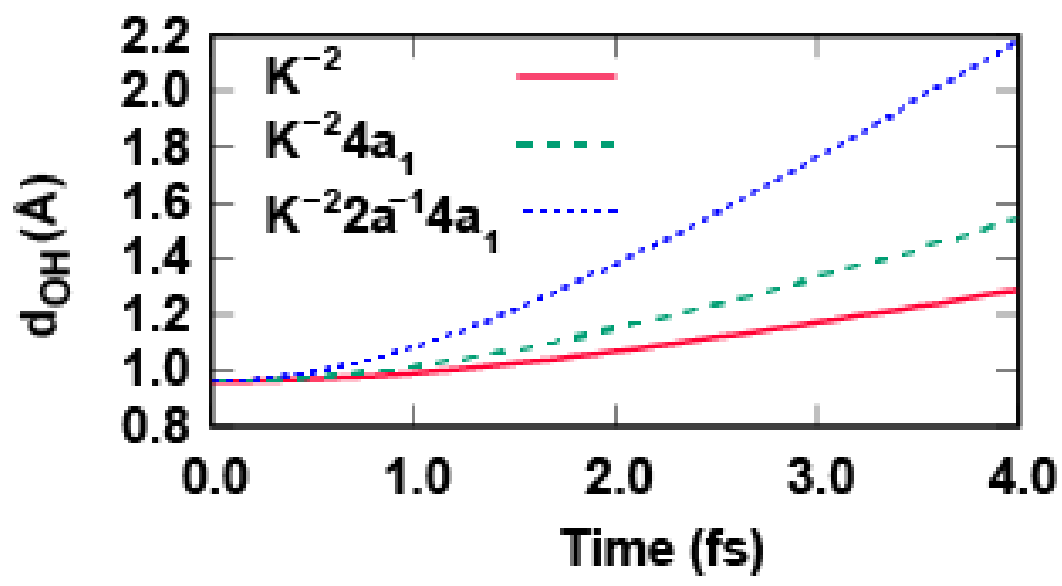

Fig.16. Evolution of the average $\mathrm{O}-\mathrm{H}$ bond distance as a function of time for three electronic states (from [57], reproduced with permission).

Experimental and theoretical results (not shown) are also available on double-core-hole spectroscopy of $\mathrm{CO}_{2}$, including a comparison with data obtained in the soft x-ray range [58].

\subsection{Direct derivation of potential energy surfaces}

The dynamical response of a core-excited molecule, in particular in the case of resonant excitation to a dissociative molecular state, depends upon the potential energy surface (PES) and the lifetime of the excited state. We have illustrated in 2.2 the core-hole-clock method, which can be used to probe the ultrafast dynamics within the core-hole lifetime in an excited species. 
Two complementary experimental tools, namely resonant inelastic x-ray scattering (RIXS) and resonant Auger electron spectroscopy (RAS), are the most useful techniques to study photoexcitation-deexcitation processes, by monitoring, respectively, x-ray photons or Auger electrons emitted during relaxation of core-excited states.

The main difference between these spectroscopies is in the electronic final states reached upon relaxation. In the case of RIXS, the molecule remains neutral with an electron in the excited orbital and a single hole, whereas in the case of resonant spectator Auger decay, the molecule becomes singly charged with an electron in the excited orbital and a double hole.

In [59] a method is demonstrated to extract information on the electron and nuclear dynamic response of molecules with double-core-hole states, created with hard x-ray radiation. In particular, in the hard x-ray regime a very effective way is found to reconstruct the PES that determines the evolution of a nuclear wave packet in the final state.

(a)
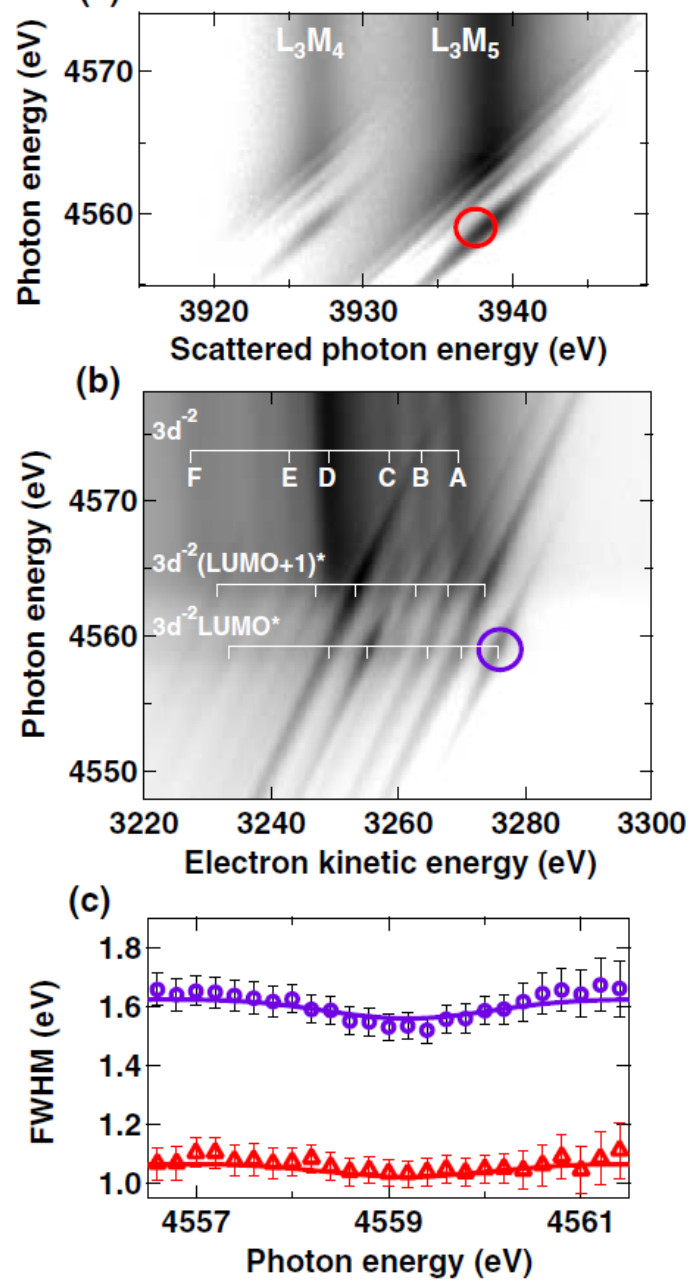

Fig.17. (a) RIXS $\mathrm{L}_{3} \mathrm{M}_{4,5}$ and (b) Auger $\mathrm{L}_{3} \mathrm{M}_{4,5} \mathrm{M}_{4,5}$ spectra recorded in $\mathrm{CH}_{3} \mathrm{I}$ near the $\mathrm{I}_{3}$ edge. (c) FWHM of RIXS emission line (triangles) and Auger line (circles) selected in (a) and (b), respectively, as a function of photon energy around the LUMO resonance (from [59], reproduced with permission).

In Figure 17 we show RIXS and RAS measurements taken for the $\mathrm{CH}_{3} \mathrm{I}$ molecule around the I 2 $p_{3 / 2}$ deep core level. The RIXS experiments have been performed at the ESRF, Grenoble, France (see [40,43] for experimental details). The resonant excitation from this level to the lowest-unoccupied molecular orbital (LUMO) occurs at $4559.2 \mathrm{eV}$. The results are shown as 2D maps, meaning that in both cases decay spectra were taken with a very small step in 
photon energy $(0.2 \mathrm{eV})$ and plotted all together with a scattered photon energy scale for RIXS (top panel) and an electron kinetic energy scale for RAS (middle panel). This method allows immediately visualizing trends for the final states as a function of photon energy.

In the bottom panel, we show the full width at half maximum (FWHM) for the spectral features marked with circles in the two panels above. The red triangles are related to a peak in the RIXS spectrum, and the blue circles to one in the RAS spectrum.

A significant difference between the two curves is the larger FWHM for the Auger line as compared to the RIXS line. This difference corresponds to different core-hole lifetime broadenings and is due to the more dissociative character of the $3 d_{5 / 2}{ }^{-2}$ LUMO Auger final state with two core holes compared to the $3 \mathrm{~d}_{5 / 2}{ }^{-1}$ LUMO RIXS final state with only one core hole.

From the experimental data, two important parameters can be extracted (see [59] for details on the theoretical method and the fitting procedure). One parameter is the lifetime broadening. It turns out that there is a factor of 2 difference in lifetime between the two final states ( 0.6 fs the $3 d_{5 / 2}{ }^{-2}$ LUMO versus 1.2 fs for $3 d_{5 / 2}{ }^{-1}$ LUMO RIXS). This difference reflects the number of the core vacancies. The lifetime is dependent upon the effective charge of the nucleus as well as on the principal quantum number of the levels involved in the relaxation.

The other parameter which could be extracted is the gradient of the potential energy curves for the excited and the final states. In Figure 18 we show the reconstructed potential energy curves for $\mathrm{CH}_{3} \mathrm{I}$ (left panel). We clearly demonstrate the dissociative character of the intermediate state as well as the two final states reached by the two different decay patterns. The same method was applied to literature data on $\mathrm{CH}_{3} \mathrm{Cl}$ for sake of comparison (right panel), with equivalent results. Such potential energy surfaces are otherwise difficult to obtain by theoretical methods for molecules containing heavy atoms, and are predictive of the dynamical behaviour of the photoexcited systems.
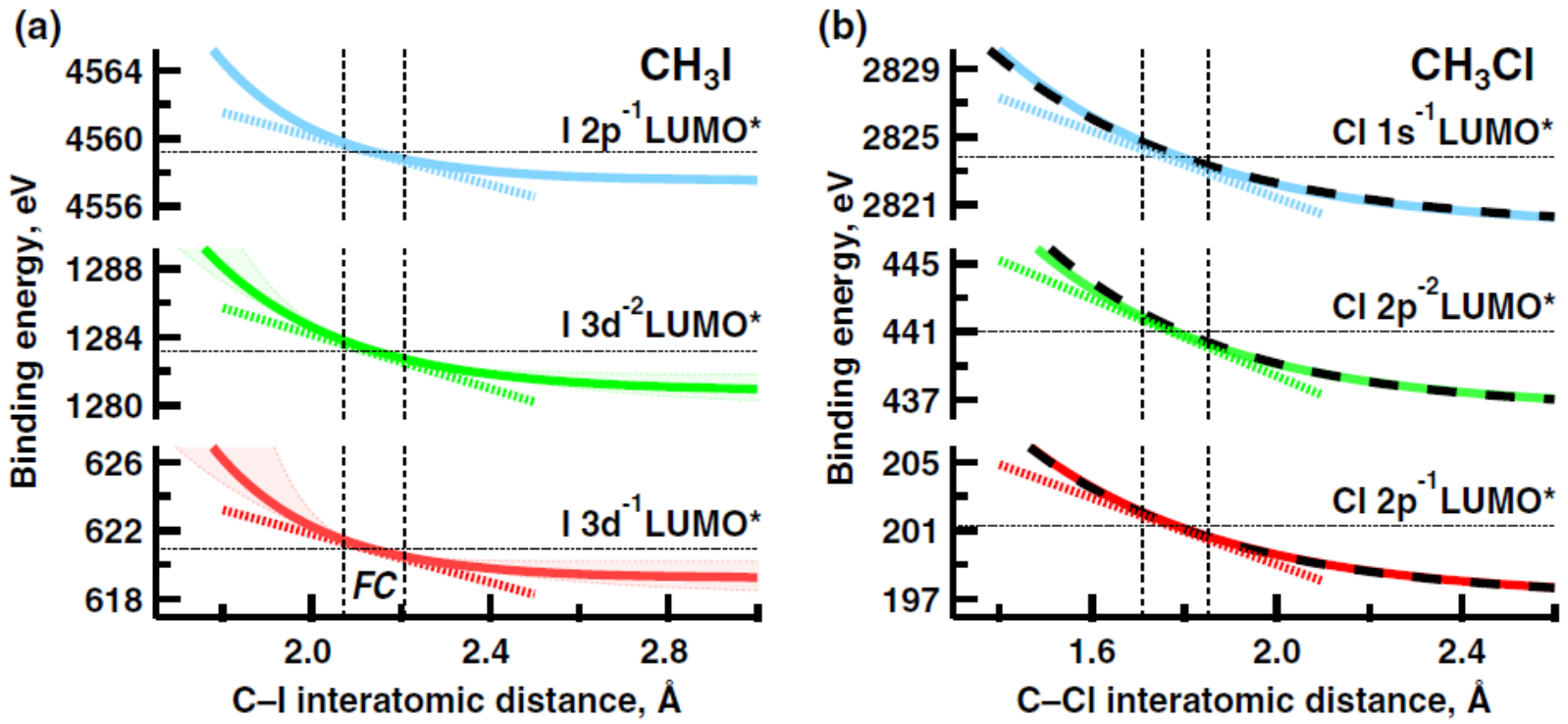

Fig.18. Potential energy surfaces reconstructed from the experimental data in (a) $\mathrm{CH}_{3} \mathrm{I}$ and (b) $\mathrm{CH}_{3} \mathrm{Cl}$ for the intermediate and final SCH and DCH states reached after RIXS or resonant Auger decay, respectively. In $\mathrm{CH}_{3} \mathrm{Cl}$ the reconstructed PES of the final states (solid lines) are compared to the results obtained with $a b$ initio calculations (dashed lines). The shaded areas in a) correspond to the uncertainty of $1 \mathrm{eV}$ for the asymptotic PES values calculated for atomic iodine (see [59] for more details) (from [59], reproduced with permission). 


\subsection{Ultrafast photodissociation in the Auger cascade following deep-core excitation}

Core-hole-clock method and effective scattering time (see 2.2 and 2.3) are concepts widely used in the soft x-ray regime, where the core-hole lifetimes for excited or ionized molecular systems are in the few-femtosecond range. Moving to the hard x-ray domain, these lifetimes became significantly shorter, in the order of 1 femtosecond or even hundreds of attoseconds. These deep core-hole states are highly unstable, and they can relax by radiative decay, i.e. emitting a photon, and/or Auger decay, i.e. emitting one electron, or more often several electrons in a cascade process (see 2.4). The Auger cascade results in the creation of multiply-charged ions, which are in turn unstable because of Coulomb repulsion, and can fragment rapidly.

Together with electronic relaxation, nuclear dynamics begins, i.e. nuclei can move away from ground-state equilibrium positions, chemical bonds begin to stretch, and, if there is enough time, they can be broken during the core-hole lifetime. This phenomenon, called ultrafast dissociation, has been studied for several molecules containing light atoms, therefore with lifetimes of few femtoseconds (see e.g. [14-20]).

In the hard $\mathrm{x}$-ray domain, the typical lifetimes are too short to allow for extensive nuclear dynamics leading to bond breaking during electronic relaxation. However, not only the first step of the electronic decay should be investigated, but also what happens during the following Auger cascade. When states with shallower core holes are reached during such cascade, they can be long-lived enough to observe dissociation on the same timescale of decay.

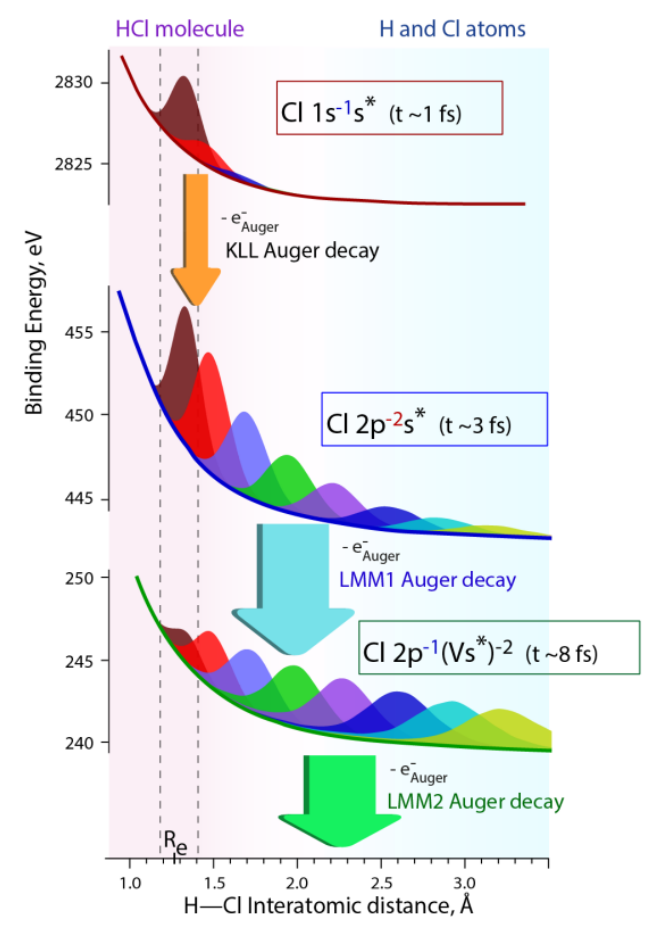

Fig.19. Potential energy curves for different steps of the KLL Auger cascade following Cl 1s $\rightarrow \sigma^{*}$ excitation. Wave-function distributions are shown in different colours for up to 8 fs after x-ray photon absorption with 1 fs increments (from [60], reproduced with permission). 
As a first example, we studied the dynamical properties of $\mathrm{HCl}$ following deep $\mathrm{Cl} 1 \mathrm{~s}$ photoexcitation and relaxation [60]. It is shown that, in the first step relaxation processes (both radiative and Auger decays) following 1s excitation, intermediate states are reached with 1 or 2 holes in shallower 2p inner shells. These intermediate states are characterized by longer lifetimes and, for the state with 2 holes, by even a stronger repulsive character of the potential energy surface. As a result, very fast nuclear dynamics leading to bond breaking is observed. In Figure 19 we show potential energy curves along the $\mathrm{H}-\mathrm{Cl}$ interatomic distance in $\mathrm{HCl}$, together with the main Auger decay pathways following $\mathrm{Cl} 1 \mathrm{~s} \rightarrow \sigma^{*}$ photoexcitation (photon energy $2823.5 \mathrm{eV}$ ). While the first step corresponds to an intermediate state too short-lived to allow significant nuclear motion, the states reached during the cascade are longer-lived and therefore ultrafast dissociation becomes possible in the subsequent steps.

In Figure 20 we show a summary of the possible decay pathways following $\mathrm{Cl} 1 \mathrm{~s}$ photoexcitation. For a heavy atoms such as chlorine, radiative decay constitute about $10 \%$ of the total relaxation process, and therefore states with one $\mathrm{Cl} 2 \mathrm{p}$ vacancy can be reached by photon emission (KL in the figure), while states with two $\mathrm{Cl} 2 \mathrm{p}$ vacancies are created by Auger electron emission (KLL in the figure). States with one or two 2p vacancies then can further decay with LMM-type Auger electron emission.

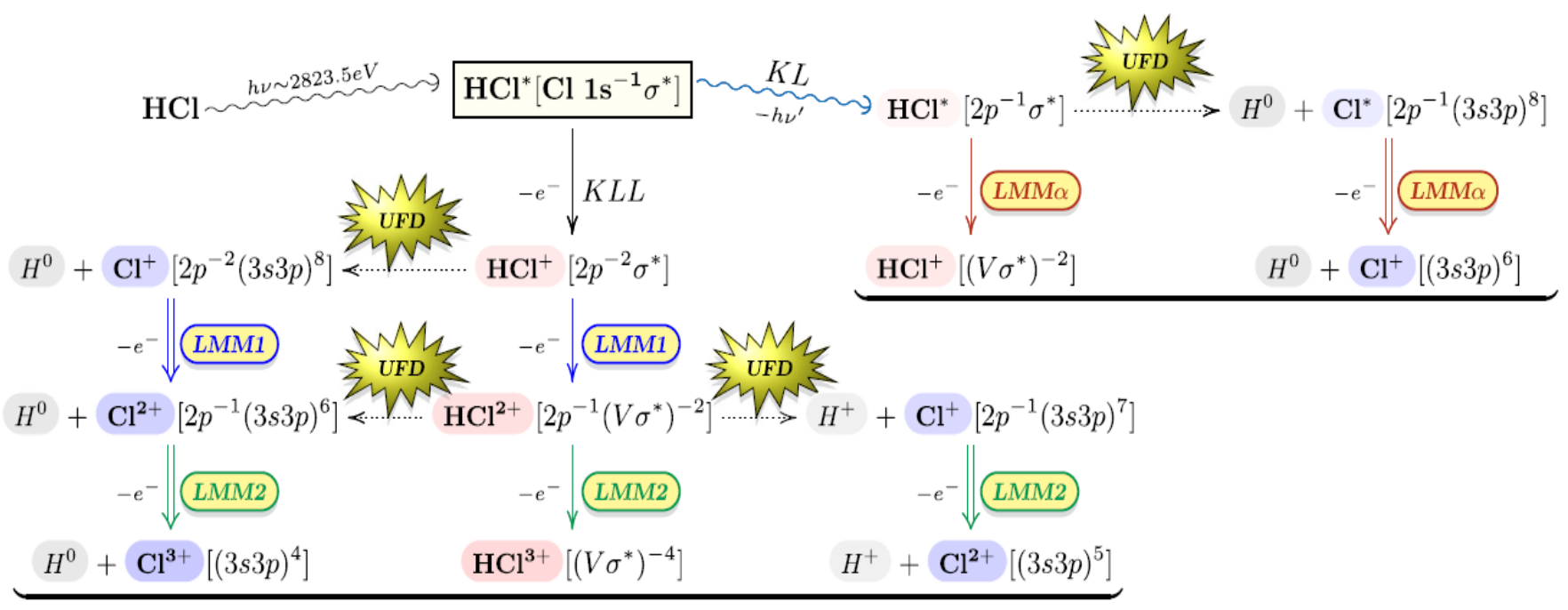

Fig.20. Diagram of the LMM Auger decays following KLL- and KL-cascade paths in $\mathrm{Cl} 1 \mathrm{~s}^{-1} \sigma^{*}$ core-excited $\mathrm{HCl}$ molecule. Single and double arrows correspond to molecular and atomic Auger decays, respectively (from [60], reproduced with permission).

Such complex decay process is reflected in the Auger emission spectra measured in the kinetic energy region of LMM decays, shown in Figure 21. Three spectral regions can be identified, labeled LMM1, LMM $\alpha$ and LMM2 respectively (see Fig.20 for the corresponding decay processes).

The top curve (b) shows the Auger spectrum taken after $\mathrm{Cl}$ 1s photoionization (photon energy $3900 \mathrm{eV}$ ) instead of photoexcitation, the middle curve (a) is the Auger LMM experimental spectrum taken after $\mathrm{Cl}$ 1s photoexcitation, and the bottom curve shows theoretical calculations in which only peaks related to the $\mathrm{Cl}$ atomic fragment, therefore to dissociation prior to decay, are taken into account. The sharp features, evident in the (a) curve and very 
well reproduced in the theoretical spectrum, correspond to the decay of atomic $\mathrm{Cl}$ produced by ultrafast dissociation taking place during 2p core-hole lifetime. Pronounced fragmentation processes at different steps of the following cascade decay are clearly enlightened.

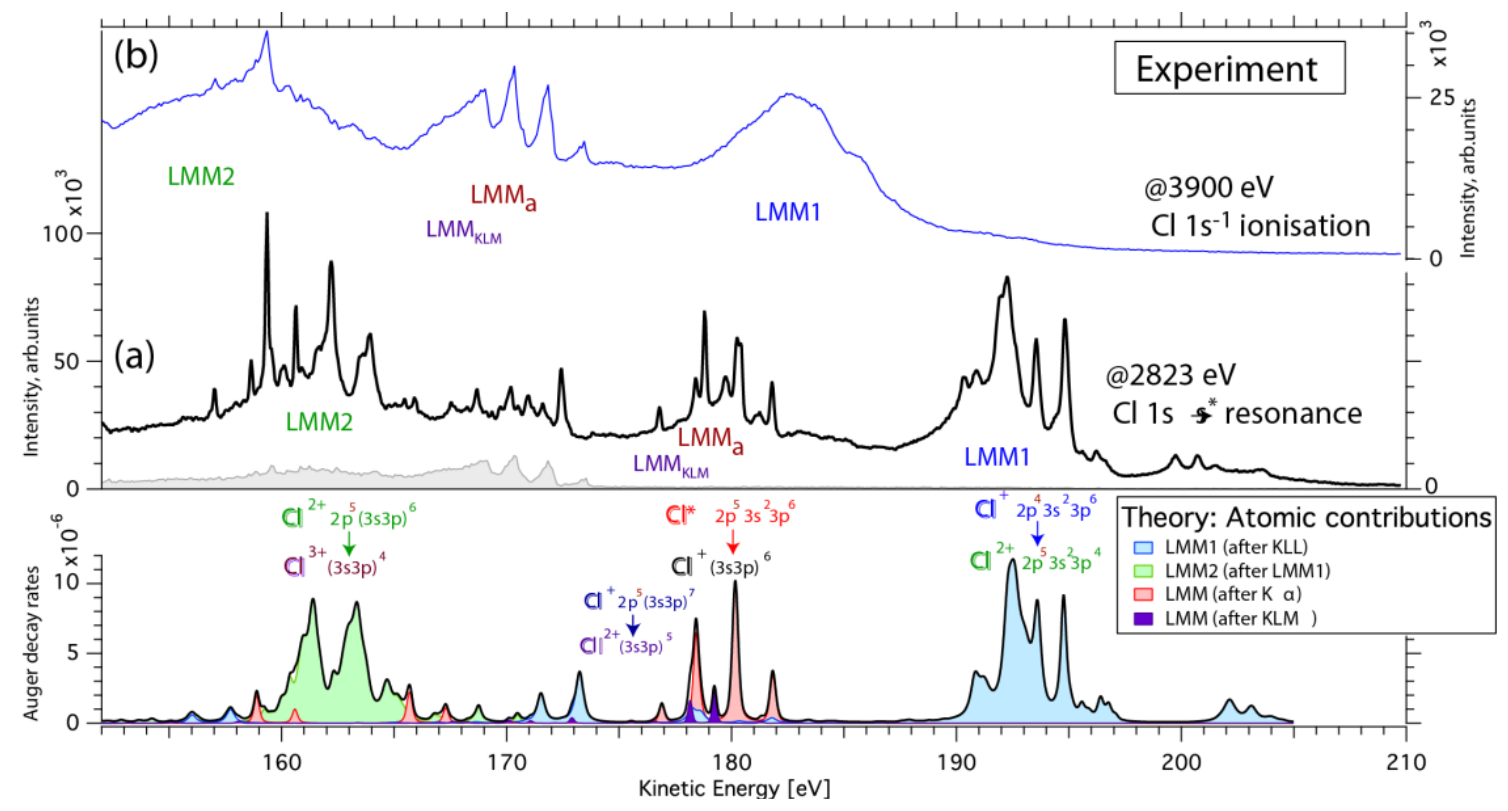

Fig.21. Top: experimental LMM Auger decay spectra of $\mathrm{HCl}$ recorded after (a) $\mathrm{Cl} 1 \mathrm{~s} \rightarrow \sigma^{*}$ excitation at 2823.5 $\mathrm{eV}$ and (b) $\mathrm{Cl} 1 \mathrm{~s}$ ionization at $3900 \mathrm{eV}$. The grey shaded curve corresponds to the "off"-resonance spectrum recorded at $2813 \mathrm{eV}$. Bottom: Calculated LMM Auger decay spectra following $\mathrm{Cl}$ 1s $\rightarrow$ 3p excitation in atomic $\mathrm{Cl}$ (black curve represents a summed total spectrum and individual contribution from different steps of the cascades are shown as filled colour curves) (from [60], reproduced with permission).

To deepen the analysis of this ultrafast fragmentation process in $\mathrm{HCl}$, the next step has been to perform measurements as a function of energy detuning from the top of the $\mathrm{Cl} 1 \mathrm{~s} \rightarrow \sigma^{*}$ resonance. In this way we can exploit the concept of effective scattering time (see 2.3) [61].

In Figure 22 we show the comparison between Auger spectra taken at a photon energy corresponding to the top of the resonance, and at a photon energy detuned by $\Omega=-2 \mathrm{eV}$ from the top. The spectra are normalized to the intensity of the spectral features related to atomic decay. It is immediately evident that the relative amount of fragmentation is a function of the distance from the top of the resonance. 


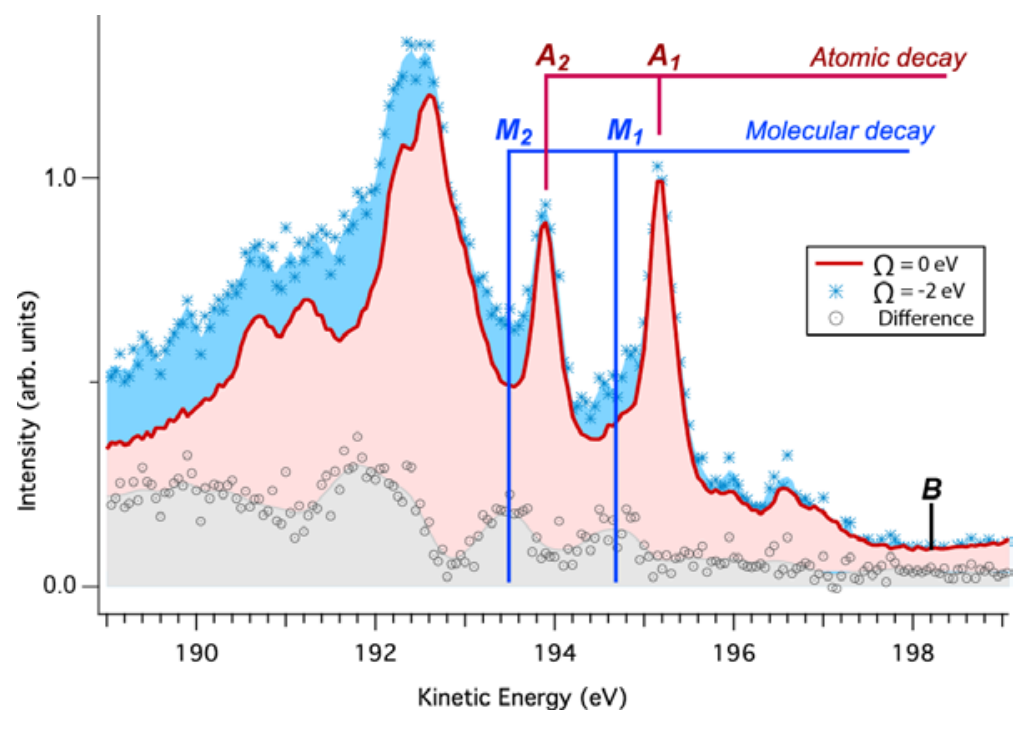

Fig.22. Effect of photon energy detuning on LMM1 Auger decay spectra recorded for gaseous HCl on top of the $\mathrm{Cl} 1 \mathrm{~s} \rightarrow \sigma^{*}$ resonance (red) and with detuning $\Omega=-2 \mathrm{eV}$ (blue). The sharp lines correspond to the atomic contributions after ultrafast dissociation in the $\mathrm{Cl} 2 \mathrm{p}^{-2} \sigma^{*}$ double-core-hole states of $\mathrm{HCl}$. The difference spectrum (grey) shows molecular contributions to the LMM1 Auger decay. The B bar marks the overall background, taken in a flat region where no spectral structures are present (from [61], reproduced with permission).

Nuclear dynamics during the cascade was modelled using a semiclassical approach: classical trajectories are started on the $\mathrm{Cl} 1 \mathrm{~s}^{-1} \sigma^{*}$ core-excited potential energy curve (PEC). Each trajectory is allowed to hop to the PECs of the $\mathrm{Cl} 2 \mathrm{p}^{-2} \sigma^{*}$ double-core-hole states according to the effective decay rate. After the first decay, the trajectories are further propagated on the new PEC and are allowed to decay according to the LMM1 lifetime $\tau_{1}$. For each calculation, 40000 trajectories were propagated until they decayed through the LMM1 Auger transition. The atomic and molecular contributions to the LMM1 Auger spectra are taken as the number of trajectories hopping at distances above and below $2 R_{e}$, respectively, where $R_{e}$ is the equilibrium bond distance in the ground state $\mathrm{HCl}$ molecule.

In order to take the detuning effects into account, the initial conditions were chosen to reproduce the cross section as:

$$
\sigma(\Omega) \propto \frac{\left|\chi_{v=0}(r)\right|^{2}}{\left[\Omega+E\left(R_{e}\right)+E(r)\right]^{2}+\frac{\Gamma^{2}}{2}}
$$

where $\chi_{v=0}(r)$ is the lowest vibrational wave function of the electronic ground state, and $E(r)$ is the PEC of the core-excited state.

In Figure 23 we show the atomic-versus-molecular ratio plotted in the resonance energy region. The experimental ratio (middle curve) does not go through a maximum exactly on-top of the resonance, as it could be expected, but it is asymmetric and peaked slightly above the resonance position. To explain this behavior, a closer look is needed at the dynamics of the decay. In particular, the different velocity of wave packets created by the photoexcitation process above and below the resonance should be taken into account.

We demonstrate that not only the PECs as a function of detuning should be considered, but also their gradient, $d E(r) / d r$. 


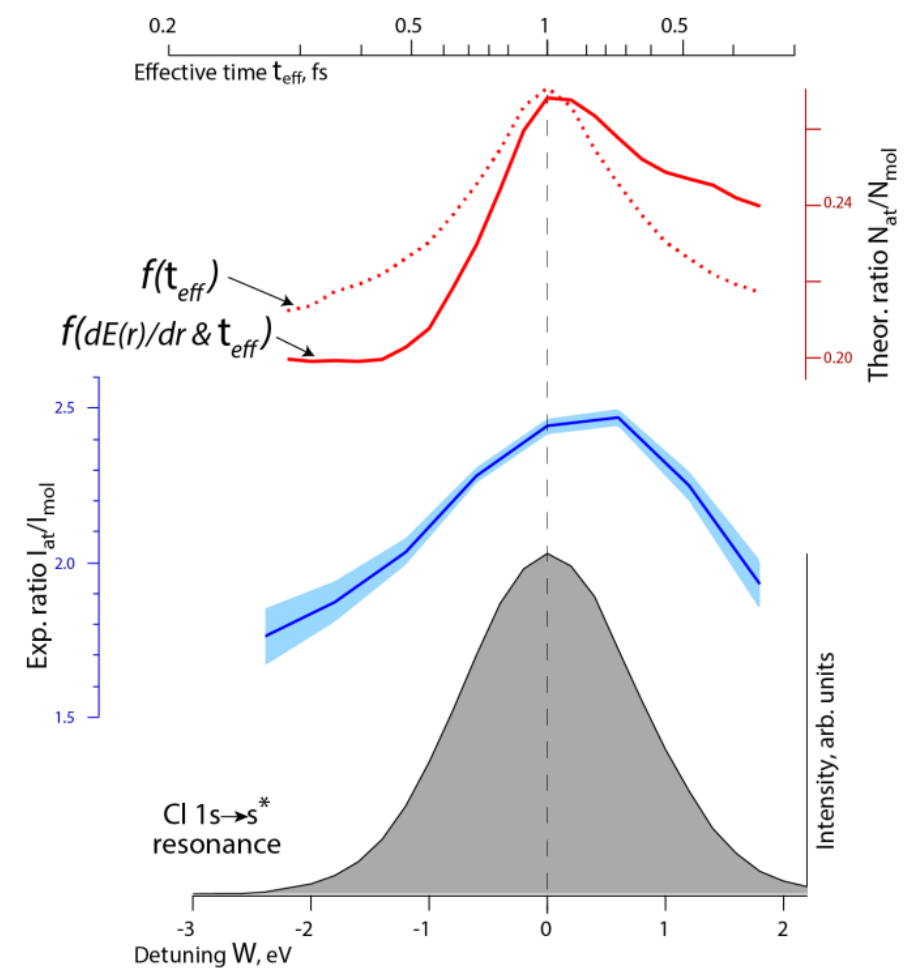

Fig.23. Middle (in blue): Experimental atomic-versus-molecular intensity ratio for ultrafast dissociation in the $\mathrm{Cl} 2 \mathrm{p}^{-2} \sigma^{*}$ double-core-hole state of $\mathrm{HCl}$ following $\mathrm{Cl} 1 \mathrm{~s} \rightarrow \sigma^{*}$ excitation using photon energies across the $\mathrm{Cl} 1 \mathrm{~s}^{-}$ ${ }^{1} \sigma^{*}$ resonance. Top (in red): Theoretical atomic-versus-molecular ratio [including both $\tau_{\text {eff }}$ and $\mathrm{dE}(\mathrm{r}) / \mathrm{dr}$, full line; only $\tau_{\text {eff }}$, dotted line]. Bottom: $\mathrm{Cl} 1 \mathrm{~s} \rightarrow \sigma^{*}$ partial electron yield spectrum (equivalent to x-ray absorption) (from [61], reproduced with permission).

In Figure 23 we show on top the theoretical curves for the atomic-versus-molecular ratio calculated without (dotted line) and with (solid line) the effect of the above mentioned potential gradients taken into account. Therefore a detailed picture of this decay process including the experimental possibility of manipulating the decay dynamics by detuning is obtained [61].

In Figure 24 we show a diagram illustrating the effect of the different energy gradients (dotted straight lines) on the wave packet dynamics at the first two steps of the KLL Auger decay cascade path for positive (blue) and negative (red) detunings. It is clear that the wave packet created for positive detunings (in blue), therefore on a zone of the potential energy curve with larger gradient, possesses large initial acceleration, and eventually takes over the wave packet created by negative detuning (in red) and reaches the bond distance at which the molecule can be considered dissociated more rapidly. Therefore a full description of the dynamics of this ultrafast fragmentation process should include the interplay between potential energy gradients and the effective scattering time, which both depend on the excitation energy. 


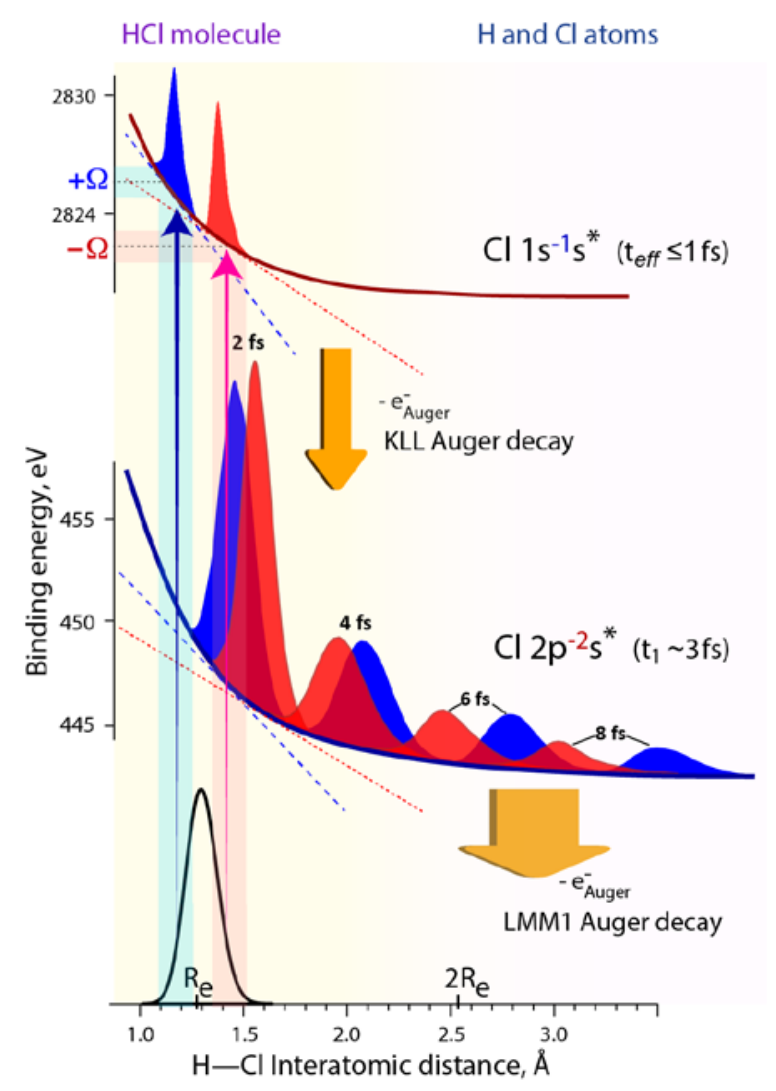

Fig.24. Diagram describing the effect of the different energy gradients (dotted straight lines) on the wave packet dynamics at the first two steps of the KLL Auger decay cascade path for positive (blue) and negative (red) detunings. The calculated initial ground state wave packet, (bottom), initially excited wave packets (top), and their distributions in the $\mathrm{Cl} 2 \mathrm{p}^{-2} \sigma^{*}$ state after 2, 4, 6, 8 fs (middle) are shown (from [61], reproduced with permission).

\subsection{Recoil due to the photoelectron's momentum}

In the hard $\mathrm{x}$-ray regime, for photoionization processes leading to the ejection of a fast photoelectron, the recoil "kick" imparted by the outgoing photoelectron to the ion left behind is far from being negligible as is the case for soft x-ray spectroscopy. On the contrary, the momentum transfer from the photoelectron to the ion induces very significant phenomena. In atoms, the only possible way to dissipate the excess recoil energy is translational motion, while for molecules additional channels include vibrational and rotational recoil. Some significant examples have been studied and will be presented for all three categories of recoilinduced effects.

\subsubsection{Translational recoil: Ne as showcase}

The role of translational recoil due to fast photoelectron emission has been studied on the showcase system neon [62]. The experimental way to enlighten it has been to measure Auger decay spectra with a selected detection geometry following deep core ionization. In particular, a strong variation in Auger lineshape was observed which was attributed to Doppler effect caused by ion translational recoil induced by the ejection of a fast photoelectron.

The philosophy of the experiment is graphically illustrated in Figure 25. 
The experiment consisted in photoionizing the Ne atom from the K-shell (ionization potential $870 \mathrm{eV}$ ), and measuring the KLL Auger electrons emitted during the decay of the core vacancy. When a photoelectron energetic enough is emitted, it gives a recoil "kick" to the ion left behind. In the specific case of Ne K-shell ionization, because of dipole selection rules, the spatial distribution of photoelectrons is shaped as a p-wave oriented along the electric-field (E) vector of the linearly polarized incoming synchrotron radiation beam.
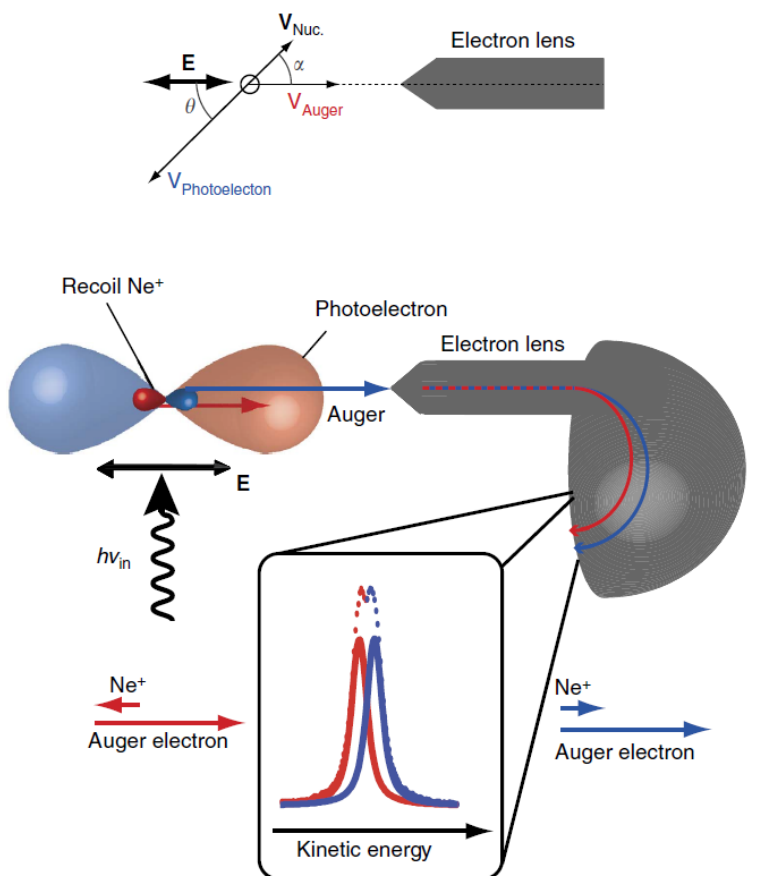

Fig.25. Schematic diagram of the physical phenomenon and of the experimental set-up. The polarization vector $\mathrm{E}$ of the incoming synchrotron radiation light is along the detection axis (from [62], reproduced with permission).

Therefore the Auger electrons ejected following the Ne 1s ionization depart from recoiling ions statistically moving in opposite directions. In our apparatus, the electron kinetic energy analyzer is mounted with the lens axis parallel to the E vector, and then the Auger electrons are collected from ions travelling towards the detector or away from it. This results in blue shift for the Auger electrons traveling towards the detector, and a red shift for the ones traveling in the opposite direction. Such Doppler shift is observed as a gradual broadening and splitting of the Auger peaks as a function of the photoelectron kinetic energy.

In Figure 26 we show Auger spectra taken for increasing values of the photoelectron kinetic energy. Only one Auger peak is shown, chosen because it is well separated, but the described behavior holds for the entire Auger spectrum. 


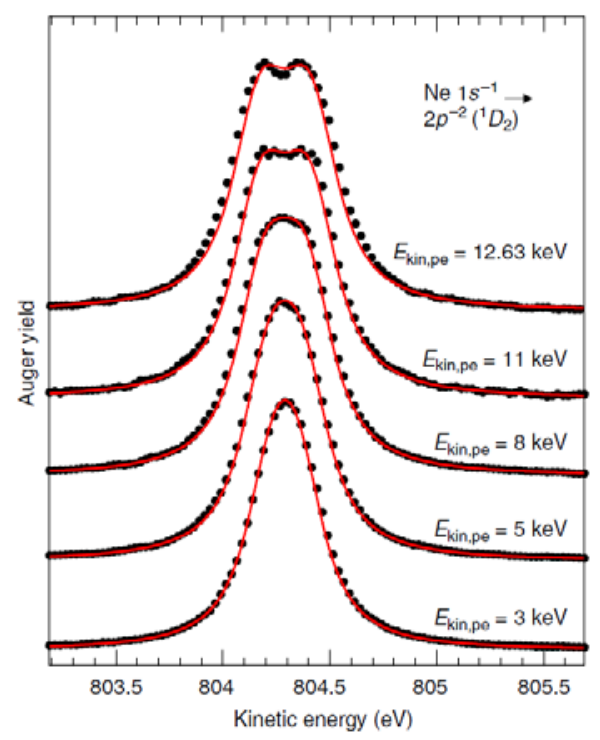

Fig.26. Ne $1 \mathrm{~s}^{-1}-2 \mathrm{p}^{-2}\left(1 \mathrm{D}_{2}\right)$ Auger transition. The spectra are measured subsequent to photoionization with kinetic energies of the photoelectron between $3 \mathrm{keV}$ and $12.63 \mathrm{keV}$ (from [62], reproduced with permission).

Clearly, the peak becomes broader with increasing kinetic energy of the photoelectron, and finally splits in two components at high photoelectron energies.

The energy difference between the red- and blue-shifted components depends on the ion recoil, since the Auger kinetic energy remains constant. This recoil energy increases with the photon energy and causes the broadening and final splitting of the Auger spectral line.

This effect, beside its importance from a fundamental point of view, can also be exploited to measure several parameters, such as photoelectron angular distribution, and degree of polarization of a light source (see [62] for more details).

The Doppler splitting observed here is simply the effect of the velocity of the emitted photoelectrons, if such velocity can be made high enough. This observation is general and does not rely on a specific aspect of photoemission dynamics. Furthermore, this Auger Doppler effect should not be limited to the present example. It has to be considered a general phenomenon in high-energy photoemission, not only for gas-phase atoms and molecules. It will have to be taken into account also in studies of other recoil effects such as vibrational or rotational recoil in molecules (see below), and can have consequences in high-kinetic-energy photoemission measurements in solids.

\subsubsection{Vibrational recoil: CO as showcase}

While in atoms the only possible dissipation of recoil energy goes to translational motion (see above), in molecules the situation is more complex. In particular, the recoil energy can be transferred not only to the translational motion, which involves "external" energy, but also into "internal motion", i.e. vibrations and/or rotations of the molecule [63]. Upon photoionization, an electron is emitted with a specific momentum, and by a simple energy conservation argument the molecular ion acquires an equal and opposite momentum, i.e. the "recoil" momentum. In general, a core-ionized level can be considered as quasi-atomic, in other words localized on a specific atomic site. Therefore the recoil momentum is initially connected to a particular nucleus, as well as to the center of mass of the entire molecule. Applying a simple total momentum conservation scheme, this momentum is shared between 
the translational recoil of the molecule as a whole and the excitations of the internal degrees of freedom, vibrational or rotational ones. In what concerns vibrational excitation, the result is an increase in the excitation probabilities of higher vibrational levels, which is superimposed to the already existing Franck-Condon excitations which are independent of the photoelectron energy.

More in detail, we consider the emission of a photoelectron with kinetic energy $E_{\text {kin }}$ and linear momentum $p_{e}$ from atom $A$ of a diatomic molecule $A B$. Because of momentum conservation, the molecule as a whole undergo a momentum change $p_{M}=-p_{e}$. As a result, the average translational energy of the molecule increases by $\Delta E_{\text {trans }}=p_{e}{ }^{2} /(2 M)$, where $M$ is the molecular mass. If the photoelectron is ejected from a core orbital, we can consider it as essentially atomic, i.e. bound to one of the two nuclei, $\mathrm{A}$ in this example. Therefore, the recoil energy of the emitter atom is $\Delta E_{A}=p_{e}^{2} /\left(2 M_{A}\right)$, and is larger than $\Delta E_{\text {trans. }}$ The energy difference between these, which we call $E_{\text {rec}}$, goes into internal excitation of the molecule, either vibrational or rotational, according to the equation:

$$
E_{\text {rec }}=\Delta E_{A}-\Delta E_{\text {trans }}=\frac{p_{e}^{2}}{2 M} \frac{M_{B}}{M_{A}}=E_{k i n} \frac{m M_{B}}{M M_{A}}
$$

where $m$ is the electron mass.

A textbook example of this effect is CO C1s photoionization, and more specifically is the change in vibrational substructure of the $\mathrm{C} 1 \mathrm{~s}$ photoelectron spectral feature as a function of photon energy, therefore of increasing photoelectron kinetic energy.
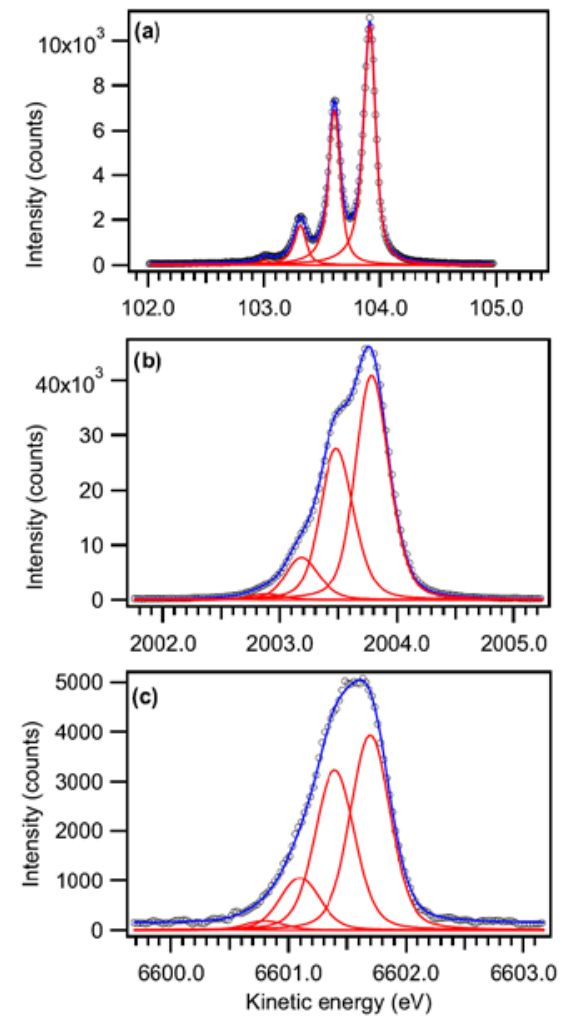

Fig.27. Carbon $1 s$ photoelectron spectra of CO at (a) $h v=400 \mathrm{eV}$, (b) $h v=2300 \mathrm{eV}$, and (c) $h v=6900 \mathrm{eV}$. Circles: experiment; red curves: vibrational peaks; blue curve: their sum (from [63], reproduced with permission). 
In Figure 27 we show the CO C 1s photoelectron spectrum taken at three different photon energies: a literature spectrum at low photon energy (soft x-rays, $400 \mathrm{eV}$ ) and two spectra with hard $\mathrm{x}$-rays (2.3 and $6.9 \mathrm{keV}$ respectively).

An obvious difference is the total spectral resolution, which worsens as the photon energy increases. However, the main effect is clearly visible, and namely the change in vibrational distribution, and in particular the increase of the probability of exciting higher vibrational levels, which is due to the transfer of recoil energy to the vibrational motion.

To render the effect more evident, in Figure 28 we plot the ratios of the vibrational levels $\mathrm{v}=1,2,3$ to the vibrational ground state $\mathrm{v}=0$. The experimental data obtained in the hard $\mathrm{x}$-ray range are the red points. The data are compared with theoretical calculations with different levels of complexity, from a simple linear coupling model to more sophisticated calculations including Morse potential, rovibrational coupling and DFT calculations (see [63] for more details). A generalized Franck-Condon factor taking into account vibrational recoil is always necessary to explain the experimental trends.

In all cases, a steady increase of the ratio is evident in the photon energy range above $2 \mathrm{keV}$, albeit with different trends (linear for $\mathrm{v}=1$, quadratic for the higher levels). All calculations are also at least able to reproduce the trends.

This is a clear demonstration of the transfer of excess energy due to recoil to internal motion, vibrational in this case.
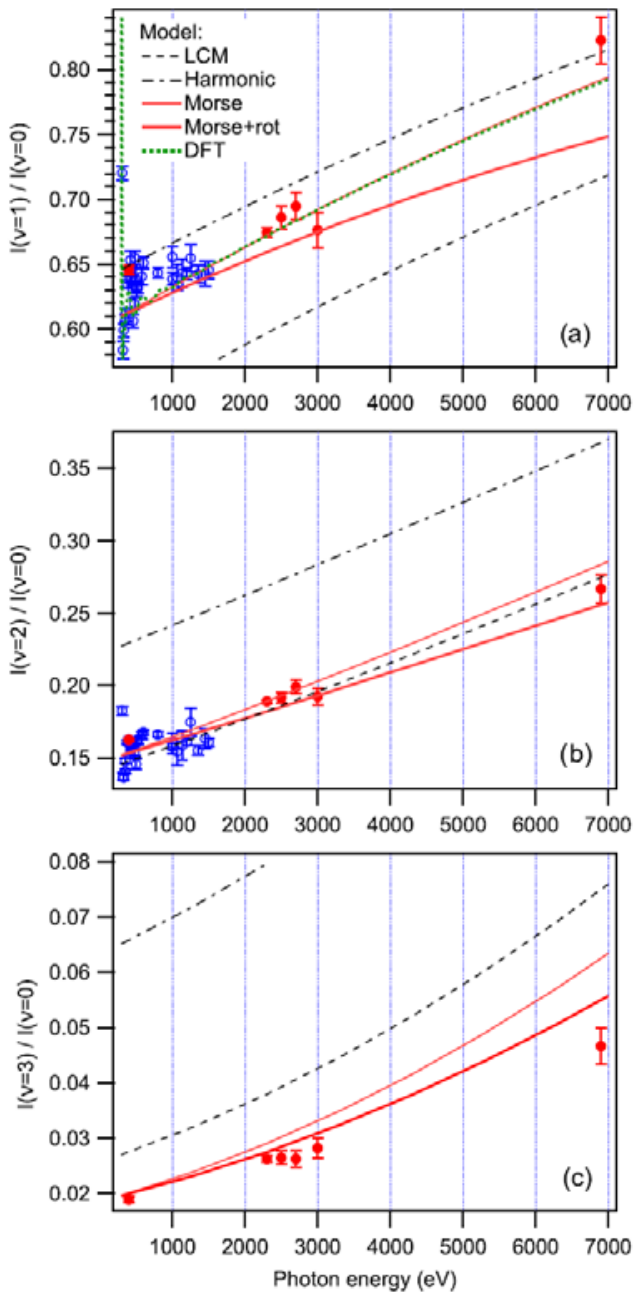

Fig.28. $v$ ratios in the $\mathrm{C} 1 \mathrm{~s}$ photoelectron spectra. Blue and red circles: measurements. Lines: various theoretical models (see [63] for more details) (from [63], reproduced with permission). 


\subsubsection{Vibrational and rotational recoil: $\mathrm{CO}$ and $\mathrm{CF}_{4}$ side-by-side}

While the effect of vibrational recoil manifests itself as a change in vibrational distribution, in particular an increase in probability of exciting higher vibrational levels, to detect rotational recoil is more subtle. The signatures of vibrational and rotational recoil effects in core-level molecular photoemission spectra are rather different. An increase of the vibrational energy can be observed as excitations of discrete vibrational levels, causing changes in the peak intensity ratios (see above). At variance with that, the spacing of rotational levels is so small that rotational excitations cannot be resolved. The only experimental parameter allowing the identification of rotational effects is a slight shift of the centroids of an individual peak towards a lower kinetic energy, which corresponds to the amount of recoil energy which goes into rotations. These recoil shifts are therefore quite hard to detect in core-level photoemission. An attempt at doing so is to use a reference system to see relative shifts.

Furthermore, the presence of rotational effects depends upon the symmetry of the molecule. A centrosymmetric molecule whose central atom is ionized will not show rotational recoil excitation, while a linear diatomic system will.

In this framework, to identify subtle rotational recoil effects in a linear diatomic molecule, again $\mathrm{CO}$, experiments were conducted on a mixture of $\mathrm{CO}$ with another system, namely $\mathrm{CF}_{4}$, which, being centrosymmetric, does not show rotational effects following the core ionization of the carbon central atom [64].

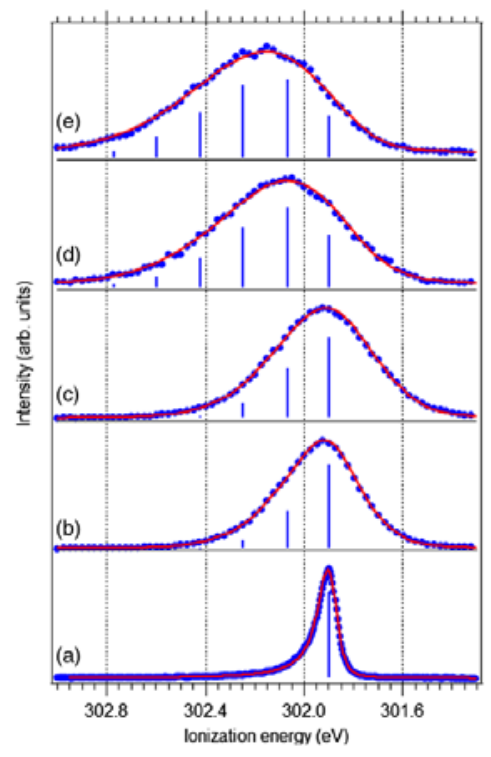

Fig.29. Carbon 1s photoelectron spectra of $\mathrm{CF}_{4}$, measured at different photon energies: (a) $330 \mathrm{eV}$, (b) $2.3 \mathrm{keV}$, (c) $3.0 \mathrm{keV}$, (d) $6.9 \mathrm{keV}$, and (e) $8.5 \mathrm{keV}$. Dots, experimental data points; continuous red line, least-squares curve-fitting result; vertical sticks, positions and relative intensities of the vibrational peaks (from [64], reproduced with permission).

In Figure 29 we show $\mathrm{C}$ 1s photoelectron spectra of $\mathrm{CF}_{4}$ taken as a function of photon energy. An interesting observation is that while for low photon energies, therefore negligible recoil, the vibrational structure consists of only one component, a full envelope develops as the photon energy, and then the photoelectron kinetic energy, increases. The excitation of higher vibrational components is entirely due to the recoil effect. As stated before, in $\mathrm{CF}_{4}$, centrosymmetric molecule excited at the central atom, no rotational effect is expected. 
Therefore it was used as a reference for a case where rotational recoil is expected, but hard to detect because its effect is only a small shift in the peak position.

In Figure 30 we show $\mathrm{C}$ 1s photoelectron spectra taken for a mixture of $\mathrm{CF}_{4}$ and $\mathrm{CO}$ for two photon energy values, 2.3 and $6.9 \mathrm{keV}$.

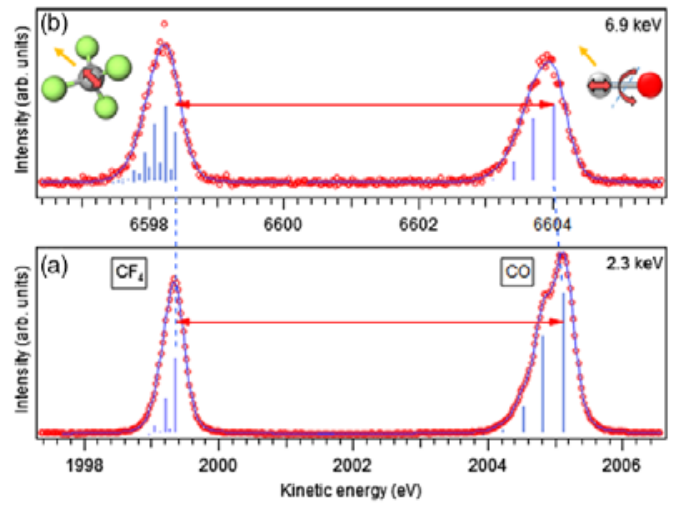

Fig.30. Carbon 1s photoelectron spectra from a mixture of $\mathrm{CO}$ and $\mathrm{CF}_{4}$ gases, measured at (a) 2.3 and (b) 6.9 $\mathrm{keV}$ photon energy. Arrows indicate the distance between the $v=0$ peaks of $\mathrm{CF}_{4}$ and $\mathrm{CO}$ (from [64], reproduced with permission).

While for $\mathrm{CF}_{4}$ the effect of increasing the photon energy, then the photoelectron energy, is only the change in vibrational distribution, for CO not only the excitation of higher vibrational components, but also a shift in peak position relative to the $\mathrm{CF}_{4}$ peak is noticeable. Since for $\mathrm{CF}_{4}$ no shift is expected, this method allows one to extract the amount of the change in centroid peak position for $\mathrm{CO}$, which would be too small to be reliably obtained from the spectrum of $\mathrm{CO}$ alone. The obtained value of this rotational shift is $90 \mathrm{meV}$.

Another interesting characteristic of $\mathrm{CO}$ is that while studying vibrational and rotational effects there is a phenomenon to take into account, and namely Coriolis coupling. Since carbon 1s ionization of $\mathrm{CO}$ results in shrinkage of the $\mathrm{CO}$ bond length between the neutral molecule and the core-ionized molecule, Coriolis coupling will cause a transfer of energy from the vibrational mode to the rotational mode.

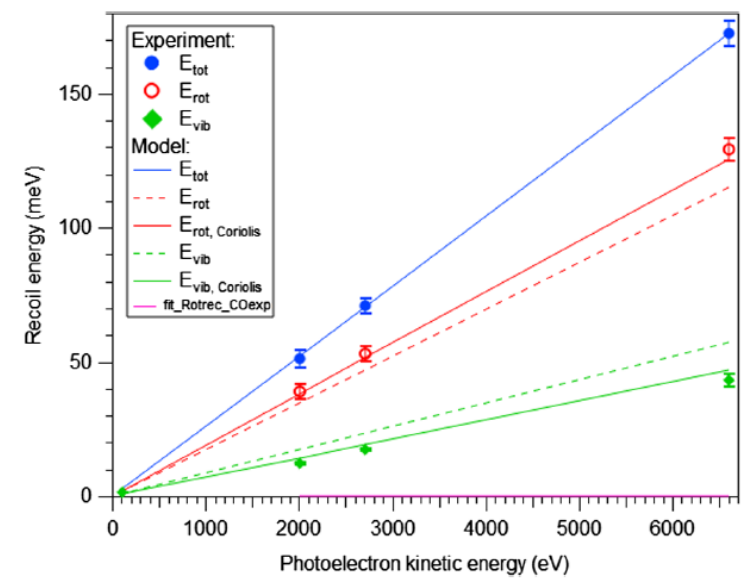

Fig.31. Recoil energy partitioning into the total internal, rotational, and vibrational recoil excitations. Experiment: solid blue circles, total recoil energy; open red circles, rotational recoil; green diamonds, vibrational recoil. Recoil-model prediction including Coriolis coupling: solid lines; from highest to lowest, total, rotational, and vibrational recoil. Model prediction without Coriolis coupling: dashed lines (from [64], reproduced with permission). 
In Figure 31 we show the recoil energy partitioning into the total internal, rotational, and vibrational recoil excitations (experimental points: blue circles, total recoil energy; red circles, rotational recoil; green diamonds, vibrational recoil). The solid lines show the recoilmodel prediction including Coriolis coupling, while the dashed lines show the model prediction without Coriolis coupling. There is clear experimental evidence of the energy transfer from vibrational to rotational excitations.

\subsection{Exotic Auger decay pathways}

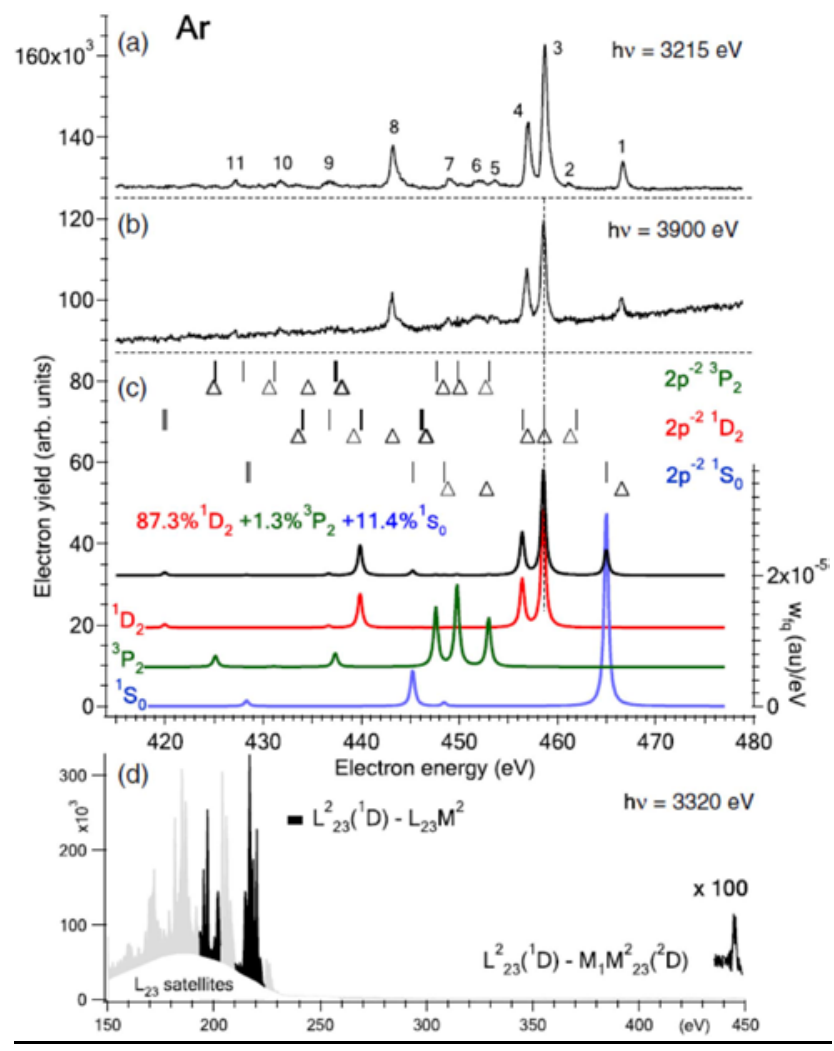

Fig.32. Observed TEOE $L_{23}^{2}-M^{3}$ spectrum in Ar with the initial double vacancy prepared by $K-L^{2}$ Auger decay following photon absorption at (a) $3215 \mathrm{eV}$ and (b) $3900 \mathrm{eV}$. (c) Calculated spectra (see [60] for details). (d) Broad range spectrum measured at $3320 \mathrm{eV}$ photon energy (from [65], reproduced with permission).

After photoexcitation or photoionization of a deep core level, the decay processes are rather complex and can proceed via several steps (the so-called Auger cascade, see 2.5). Starting from a deep core hole, the first step can be the creation via Auger emission of two shallower core holes. This double-core-hole state then decays further. The most likely decay mode of a double vacancy is sequential relaxation, i.e., the core holes decay one at a time, passing through a single-core-hole intermediate step.

Another less explored possibility is that such decay takes place in only one step, and namely that two electrons fill the two core holes simultaneously and only one Auger electron is emitted. Such two-electron one-electron (TEOE) transitions give rise to peaks falling at higher kinetic energy with respect to the spectral lines of the sequential Auger decay. 
We have studied the prototypical system Ar and obtained a well-resolved $\mathrm{L}_{23}^{2}-\mathrm{M}^{3}$ TEOE spectrum [65]. We have also extracted the branching ratio for this TEOE process with respect to the more probable sequential Auger decay of the $L^{2}{ }_{23}\left({ }^{1} D_{2}\right)$. The $\mathrm{L}^{2}$ core-hole states originated from a K-shell ionization and subsequent $\mathrm{K}-\mathrm{L}^{2}$ decay. The TEOE spectra were measured in the 415-480 eV kinetic energy range and are shown in Figure 32.

The TEOE decay is a minor relaxation pathway of the $L_{23}^{2}$ vacancy with a $2.2(4) \times 10^{-3}$ branching ratio. The experimental spectra are very well reproduced by the second-order perturbation model when the population of initial double core holes is taken into account (see [65] for details on the modelling of the TEOE spectra). Finally, the application of TEOE spectroscopy to other targets is possible and increased chemical sensitivity is expected when valence electrons take part in the decay.

\subsection{Novel interference phenomena}

The excitation of a core electron to an empty orbital or to the ionization continuum creates core-hole states which are then filled rapidly by radiative (photon emission) or nonradiative (Auger electron emission) decay processes. In general, the deeper the core hole is, the shorter its lifetime, and as a consequence the larger the natural line broadening of the corresponding spectral features in an absorption spectrum. If the energy separation between peaks corresponding to core-excited states is of the same amount as their line width, interferences between several decay channels concerning overlapping excited states and leading to the same final states are possible.

A schematic view of such effect is presented in Figure 33 [66].

In the soft x-ray regime, an analogous effect called lifetime-vibrational interferences (VLI) in the resonant Auger spectra has been reported (see e.g. [67,68]. In that framework, the spacing between vibrational levels is of the same order of magnitude as the lifetime broadening, making the interference possible.

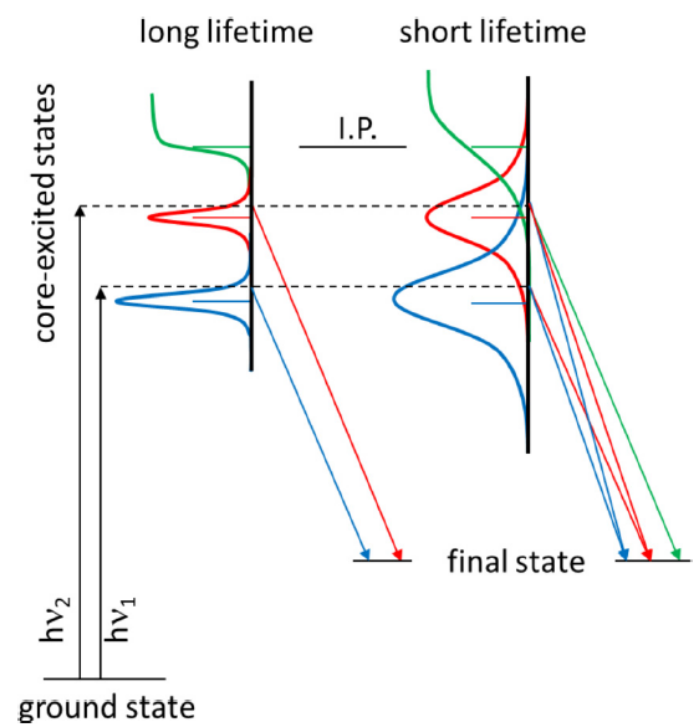

Fig.33. Schematics of the core-electron photoexcitation by $h v 1$ or $h v 2$ photons, and resonant-Auger decay mechanisms. In case the energy splitting between two core-excited states is small enough and the broadening large enough (right part of the figure), the use of a narrow excitation bandwidth can lead to a coherent excitation of several excited or ionized states, which will be reflected in the population of a specific final state. The opposite case is described on the left part of the figure (from [66], reproduced with permission). 
The electronic state-lifetime interference (ELI) phenomenon was at first studied in two prototypical systems, argon and xenon [66,69,70]. The main signatures of interference phenomena are shift in the energy position and asymmetry of the spectral features related to the final states of the Auger decay. Furthermore, for the absorption process a model was derived to extract the intensities of the transitions to the resonant intermediate states. For decay processes, the probabilities of different decay channels was determined (spectator Auger, shake-up and shake-down processes) (see [65] for details).

This research line was then extended to molecules, in particular with the purpose of comparing the effect in two chlorine-containing systems, $\mathrm{HCl}$ and $\mathrm{CH}_{3} \mathrm{Cl}$, around the $\mathrm{Cl} 1 \mathrm{~s}$ threshold [71].

In general, the cross section for resonant Auger decay is expressed by the KramersHeisenberg formula:

$$
\sigma\left(\omega, \omega^{\prime}\right) \propto\left|\sum_{c} \frac{\langle f|Q| c\rangle\langle c|D| o\rangle}{\omega-\omega_{c o}+\frac{i \Gamma}{2}}\right|^{2} \delta\left(\omega-\omega^{\prime}-\omega_{f o}\right)
$$

This is in analogy with eq.(2), with the main difference that for Auger decay the FranckCondon factor for intermediate and final states contains the Coulomb operator instead of the dipole operator as in the RIXS formalism. The wavefunctions for the ground, intermediate and final states are a product of electronic and vibrational functions, so the cross section can be rewritten as:

$$
\sigma\left(\omega, \omega^{\prime}\right)=\sigma_{D I R}(\omega)+\sigma_{E L I}(\omega)+\sigma_{V L I}(\omega)+\sigma_{E L I, V L I}(\omega)
$$

Here $\sigma_{D I R}(\omega)$ describes the direct term which corresponds to a decay without interferences. The contributions $\sigma_{E L I}(\omega), \sigma_{V L I}(\omega)$ and $\sigma_{E L I, V L I}(\omega)$ originate from the cross terms and describe ELI, vibrational lifetime interferences (VLI), and a mixture of electronic and vibrational lifetime interference, respectively.
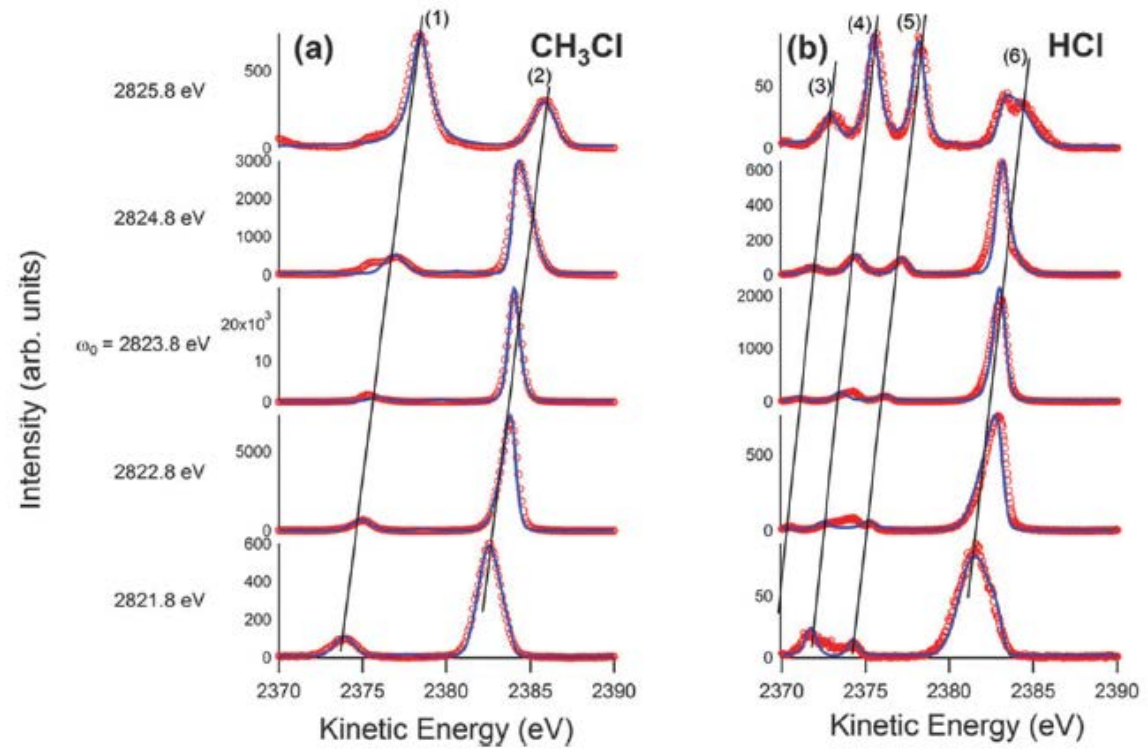

Fig.34. Auger spectra of $\mathrm{CH}_{3} \mathrm{Cl}$ (a) and $\mathrm{HCl}$ (b) for several photon energies around the $1 \mathrm{~s}^{-1}$ LUMO resonance. The dots represent the experimental points and the solid lines result from our simulation (see [71] for a detailed assignment) (from [71], reproduced with permission). 
On these grounds, a model was developed to describe the effect of ELI on the position and shape of resonant Auger spectral features (see [71] for details).

In Figure 34 we show resonant Auger decay spectra for both molecules around the Cl 1sLUMO resonance. It is immediately evident that the dispersion of the spectral lines is not exactly linear, as it would be expected in absence of the electronic state-lifetime interference. To better visualize the phenomenon, in Figure 35 we show on top the absorption spectrum for $\mathrm{HCl}$, and then the pseudo cross sections for some of the final states reached after Auger decay. We use the term "pseudo cross section" because in our apparatus the spectra are taken with the analyser parallel to the direction of the polarization vector, therefore angular effects are not taken into account.

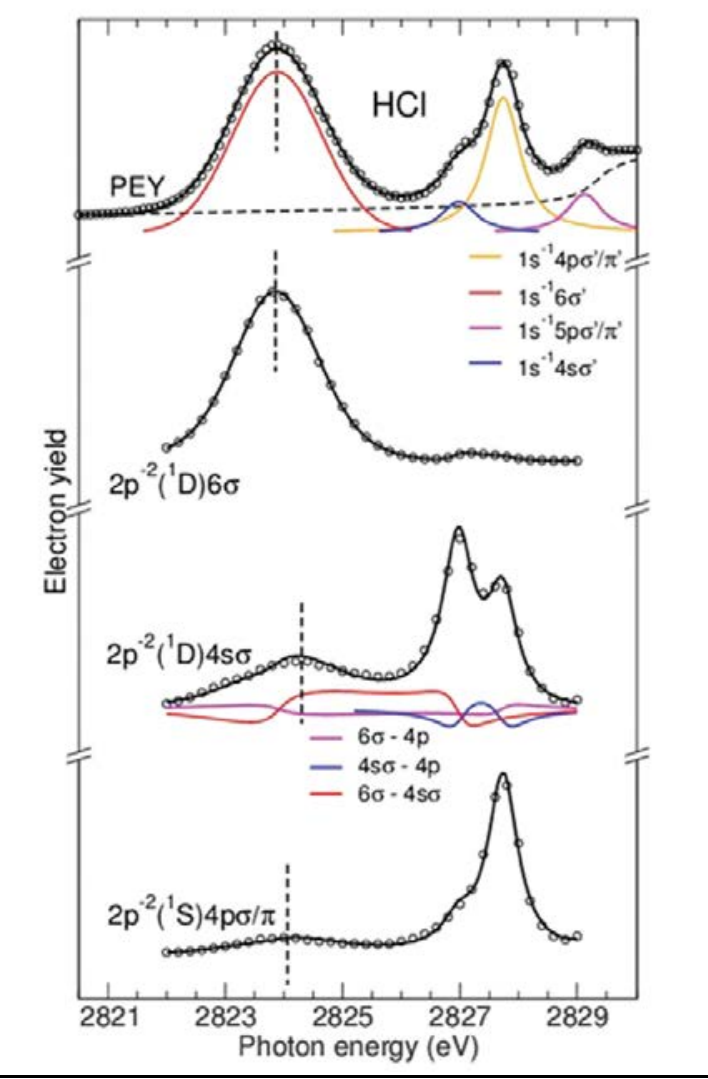

Fig.35. PEY (photoelectron yield) spectrum, analogous to absorption, as well as the pseudo-cross sections leading to the final states $2 \mathrm{p}^{-2} 6 \mathrm{~s}, 2 \mathrm{p}^{-2} 4 \mathrm{~s} \sigma$, and $2 \mathrm{p}^{-2} 4 \mathrm{p}$ in $\mathrm{HCl}$. The subspectra below the PEY spectrum indicate the contributions of the individual $\mathrm{Cl}_{1 \mathrm{~s}^{-1}}$ core excited states and the subspectra below the cross section of the $2 \mathrm{p}^{-2} 4 \mathrm{~s} \sigma$ final state show the ELI contributions (from [71], reproduced with permission).

It is clear that in correspondence of the transition to the LUMO in the absorption spectrum the maxima of the cross sections of the final states fall in different positions. This finding is in fact the signature of the electronic state-lifetime interference. Similar results have been obtained for $\mathrm{CH}_{3} \mathrm{Cl}$ (not shown). This phenomenon can be considered a general effect to be taken into account for photoexcitation of both atoms and molecules in the hard $\mathrm{x}$-ray regime. 


\subsection{Electron-recapture and post-collision interaction effects}

The dynamics of photoelectron and Auger emission and their interplay was studied with two different experimental setups, namely HAXPES (see 3.2.1) for high-resolution photoelectron and Auger measurements, and CELIMENE (see 3.2.2) for electron-ion coincidence investigations.

\subsubsection{Single-channel Auger measurements}

The phenomena of PCI (post-collision interaction) and electron recapture are summarized in 2.4. Here we show experimental evidence obtained by measuring Auger spectra following deep core ionization in a showcase system, argon [72]. The measurements have been performed with the HAXPES setup.

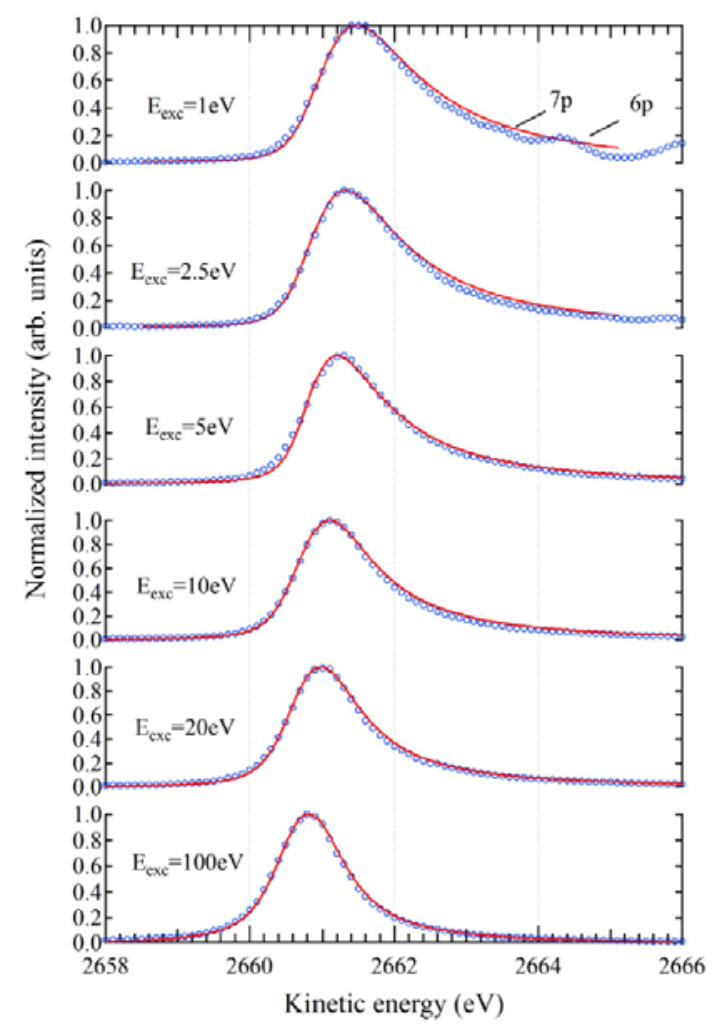

Fig. 36. Measured (blue circles) and calculated (red lines) $K L_{2,3} L_{2,3}\left({ }^{1} D_{2}\right)$ Auger line profiles for selected excess energies $\left(E_{\text {exc }}\right)$ above the Ar $1 s$ ionization threshold (from [72], reproduced with permission).

In Figure 36 we show the Auger spectrum for one specific final state reached after decay of the Ar 1s vacancy, namely the $K L_{2,3} L_{2,3}\left({ }^{1} D_{2}\right)$ one. The spectra are reported as a function of the photoelectron excess energy above the ionization threshold. It is evident that the line profile is much distorted close to threshold, and becomes more and more symmetric as the energy distance from threshold is increased.

To better visualize the effect, in Figure 37 we show the shift of the same peak as a function of the excess energy. It is clear that the effect vanishes completely quite far from threshold, and therefore that the change in Coulomb potential which induces it is "felt" by the Auger electron for an extended photoelectron energy interval. 


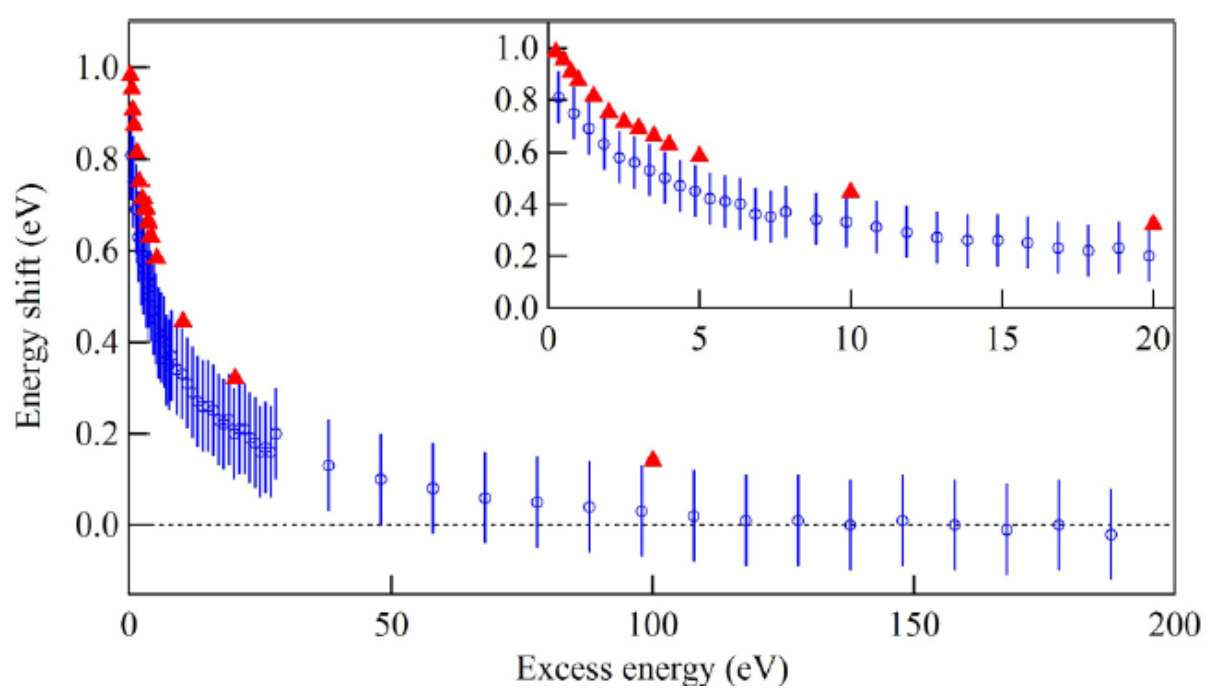

Fig.37. Measured (blue circles with error bars) and calculated (red triangles) line shift as a function of excess energy $E_{\text {exc }}$ above the ionization threshold for the $K L_{2,3} L_{2,3}\left({ }^{1} D_{2}\right)$ Auger line (from [72], reproduced with permission).

Another interesting phenomenon originating from the ejection of a slow electron and the subsequent fast Auger emission is the electron capture. In Figure 38 we show the Auger spectra around the Ar 1s threshold under the form of a 2D map.

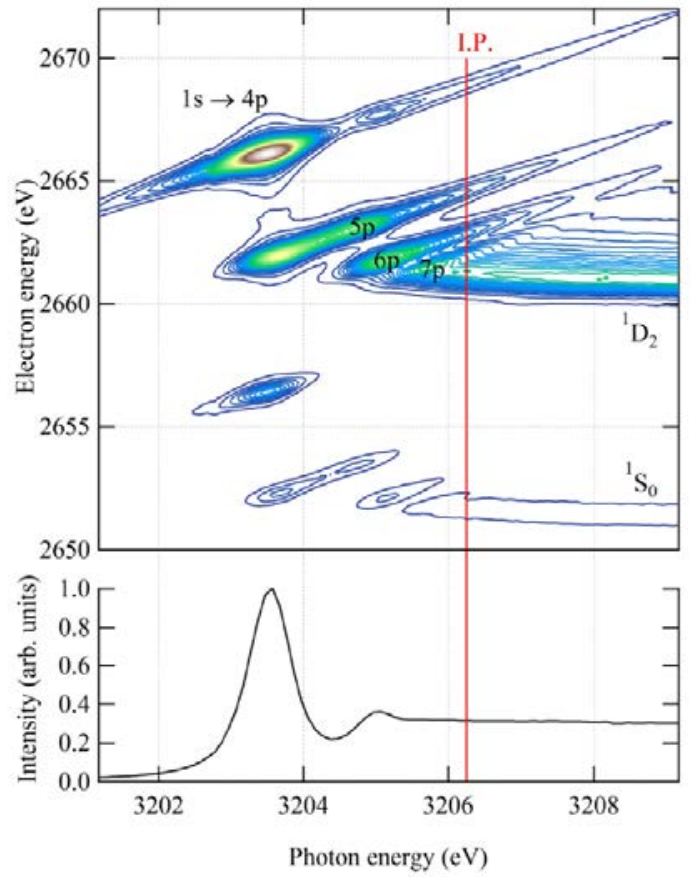

Fig.38. (Top) $K L_{2,3} L_{2,3}$ Auger relaxation of 1 s core-excited argon represented as a 2D map showing the Auger electron energy as a function of incident photon energy. The different excitations to discrete electronic states are indicated below threshold and final ionized states above. (Bottom) Absorption spectrum of Ar around the $1 s$ ionization threshold, I.P., indicated as a vertical solid line (from [72], reproduced with permission). 
Some of the states of the form $2 \mathrm{p}^{-2} \mathrm{np}$, reached below threshold by resonant Auger decay, are present also above the ionization threshold. Such behavior is the signature of electron capture: a slow photoelectron is emitted, the Auger electron overtakes it, and due to PCI the photoelectron loses energy which is gained by the Auger electron. If the exchanged energy is more than the initial energy of the photoelectron, the latter bears negative energy and can be recaptured in a discrete state.

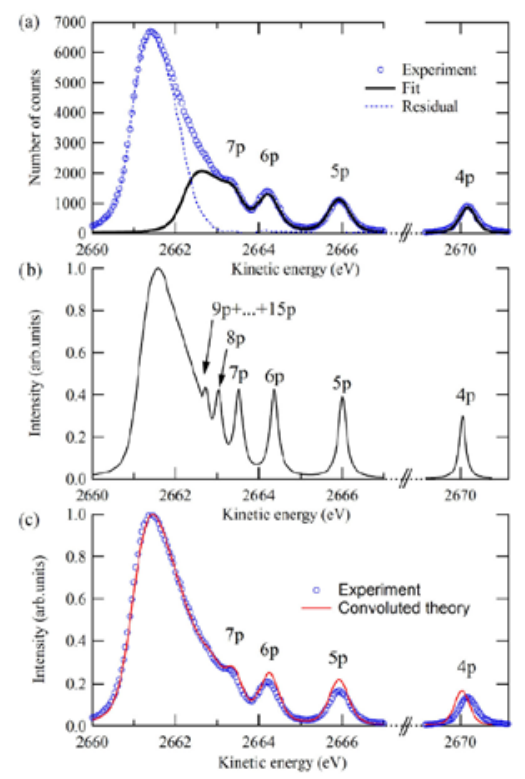

Fig.39. Experimental and calculated Auger spectrum at excess energy $E_{\mathrm{exc}}=1 \mathrm{eV}$ above the Ar 1 s ionization threshold. (a) Experimental spectrum (blue circles) with a tentative fit to the individual contributions from discrete Rydberg states (see [67] for details). (b) Calculated Auger spectrum with recapture to discrete $n p$ states with $n=4-15$. (c) Convoluted theoretical spectrum compared to the experimental spectrum (blue circles) (from [72], reproduced with permission).

In Figure 39 we show experimental and calculated Auger spectra for an excess energy of 1 $\mathrm{eV}$. Structures related to discrete excited states are clearly visible.

To gain further information about the nature of the recapture process, individual cross sections were measured and calculated for all excited states of the form $2 p^{-2} n p$ with $n=4-7$. The results are reported in Figure 40.

It can be noticed that for low excess energy the photoelectron recapture occurs mainly in the $n p$ states with $n=5-8$. Furthermore, the trend of the cross sections with excess energy above threshold is state-dependent, showing a steep decrease for the higher states.

This behaviour is due to the overlap of the radial wave function of the emitted photoelectron in the intermediate and final discrete states.

Therefore in [72] a rather complete picture is given of the interaction between the photoelectron, the Auger electron, the photoelectron, and the Coulombic field of the ion left behind. We show that the position and lineshape of the Auger peaks is strongly affected by PCI. Furthermore, the energy exchange between the photoelectron and the Auger electron can even cause the photoelectron to slow down to the point that a recapture is possible and some discrete Rydberg excited states can be populated even above an ionization threshold.

We believe that this approach is general, and can be applied to the investigation of PCI effects in other atomic and molecular systems, especially for ionization of very deep shells where these effects shall be even stronger. 


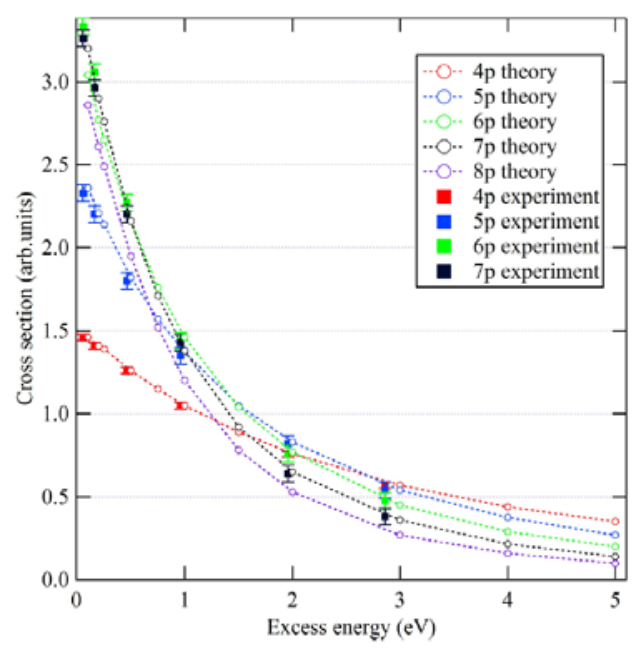

Fig.40. Experimental and calculated individual recapture cross sections for discrete Rydberg states $n=4-8$ as a function of excess photon energy above the Ar $1 s$ ionization threshold (from [72], reproduced with permission).

\subsubsection{Electron-ion coincidence studies}

While with high-resolution photoelectron and Auger spectroscopy a detailed picture of the energetics of PCI effects can be achieved, a complementary technique is electron-ion coincidence, which allows enlightening the dynamics of the processes by connecting the photoelectron emission to a specific ionic state.

In Figure 41 we show the possible decay pathways in argon following 1s photoionization (see [73] and references therein for details). The measurements have been performed on the SOLEIL beam line LUCIA with the CELIMENE setup (see 3.1.2 and 3.2.2)

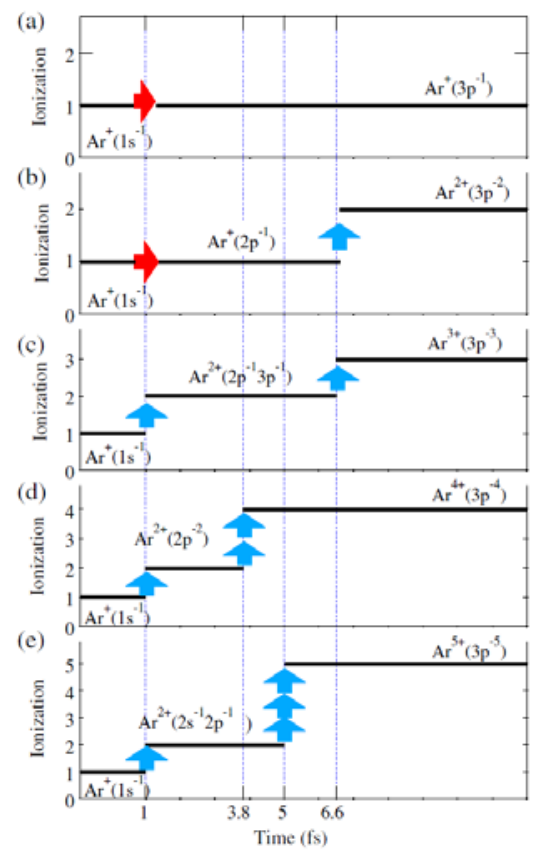

Fig.41. Decay pathways leading to the production of $\mathrm{Ar}^{\mathrm{n}+}$, with $\mathrm{n}=1-5$. The horizontal arrows in panel (a) and (b) represent $\mathrm{K} \beta$ and $\mathrm{K} \alpha$ radiative decays, respectively. Vertical arrows represent Auger decays (from [73], reproduced with permission). 
The red arrows in the figure indicate radiative decay $(\mathrm{K} \alpha$ or $\mathrm{K} \beta$ ), and the blue arrows mark Auger decay processes. To form $\mathrm{Ar}^{+}$, the only possible pattern is radiative decay, while for all other charge states at least one step consists of Auger electron emission.

In Figure 42 we show electron-ion coincidence measurements taken for photoelectron energy of $2 \mathrm{eV}$, thus very close to the ionization threshold. The experimental data are reported together with theoretical calculations [73].

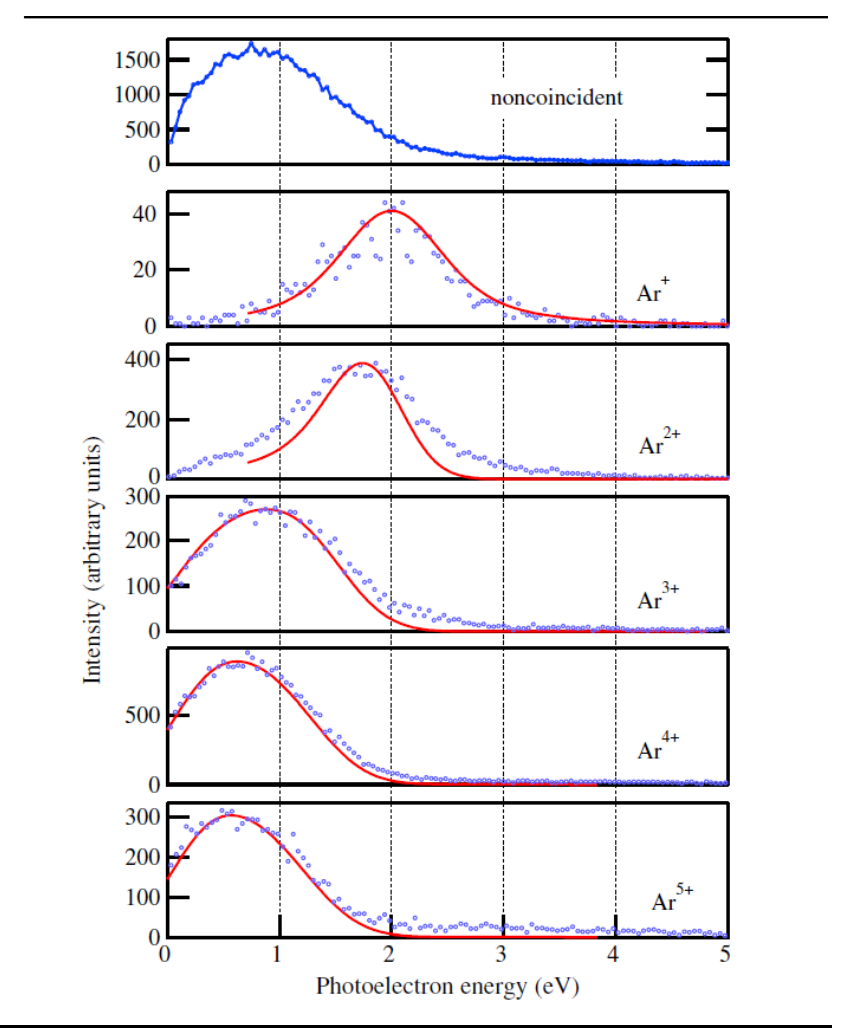

Fig.42. Experimental (circles) and theoretical (solid) partial photoelectron spectra measured in coincidence with $\mathrm{Ar}^{\mathrm{n}+}$ ions $(\mathrm{n}+1-5)$ for excess energy $2 \mathrm{eV}$ above IP. The top curve shows the noncoincident photoelectron spectrum (from [73], reproduced with permission).

The spectrum in coincidence with $\mathrm{Ar}^{+}$shows no shape distortion, while all other spectra taken in coincidence with higher charges $\left(\mathrm{Ar}^{2+}\right.$ through $\left.\mathrm{Ar}^{5+}\right)$ exhibit asymmetric line profiles and energy shifts which are the clear signature of PCI effects.

The rationale behind is the following: the asymmetry of the spectra depends on the charge of the residual ion, $\mathrm{n}^{+}$: the larger the charge $\mathrm{n}$, the larger the shift and asymmetry of the line shape. After the creation of the 1s vacancy, Auger electrons are emitted with high kinetic energy (in the hundreds to thousands of $\mathrm{eV}$ range). They rapidly overtake the slow photoelectron. Therefore the ion field "felt" by the photoelectron changes during the course of the Auger decay. After 1s photoionization, the photoelectron "feels" the field of $\mathrm{Ar}^{+}$. After the first Auger decay, it is affected by the field of $\mathrm{Ar}^{2+}$, then as the Auger decay cascade proceeds $\mathrm{Ar}^{3+}$ etc. Since then the photoelectron experiences a stronger and stronger Coulomb field, it decelerates (see the energy shift as a function of increasing ion charge in fig.42).

We conclude that the technique used, electron-ion coincidence, is able to shed light on subtle details of the photoelectron-Auger-ion interaction following deep core ionization. 


\subsection{Disentangling complex decay patterns after deep-core-hole ionization}

Absorption of an x-ray photon by a heavy atom can ionize or excite an electron from a deep shell, creating an electronic state with an inner hole which is highly unstable and decays. Electronic relaxation can involve complex multistep cascades, including radiative (photon emission) and nonradiative (Auger electron emission). Furthermore, deep core-hole states have very short lifetimes, on the femtosecond time scale or even shorter. As a consequence, phenomena like the ones described in the previous sections (electronic-lifetime interference, PCI, electron recapture) are likely to occur,

In [74], a joint experimental and theoretical investigation is reported of relaxation cascade processes following $1 \mathrm{~s}$ photoionization in a prototypical system, argon. While different spectroscopic techniques can give an accurate description of some aspects of such a complex process, we use a suitable unprecedented combination of several of those. We demonstrate that such combination is crucial in gathering information on the interplay of all possible pathways, and therefore in deriving a complete picture of the overall phenomenon.

The techniques used are photon-ion coincidence and photoelectron-ion coincidence measurements with the CELIMENE setup on the LUCIA beam line, and high-resolution Auger spectroscopy with the HAXPES setup on the GALAXIES beam line.

In Figure 43 we show a direct comparison between the two coincidence techniques, which enlightens the complementary information which can be gathered.
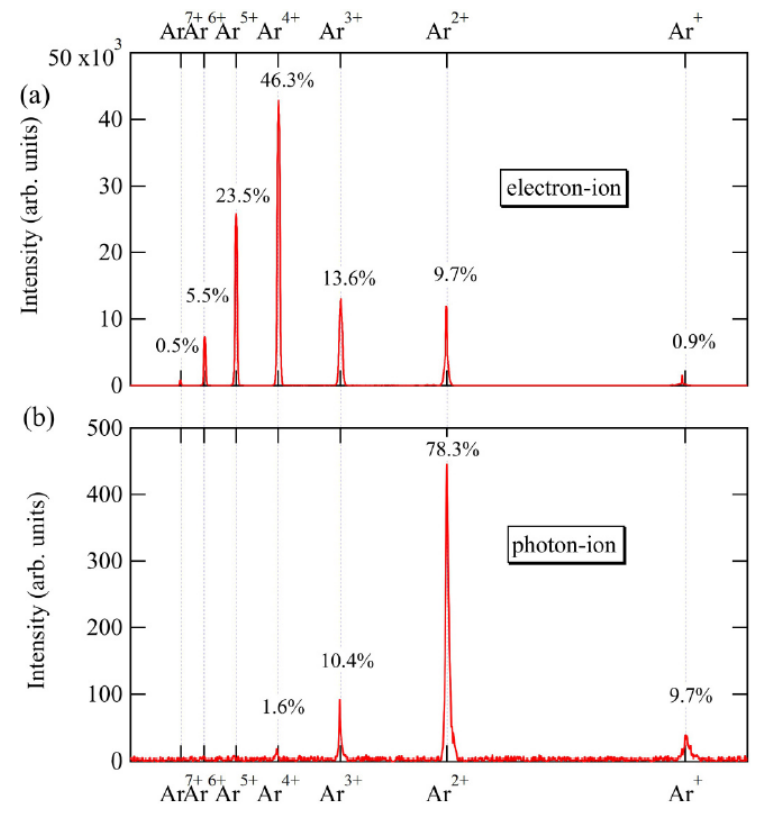

Fig.43. Coincidence spectra measured $6 \mathrm{eV}$ above the argon 1s ionization threshold: (a) photoelectron-ion coincidences, (b) photon-ion coincidences (from [74], reproduced with permission).

The two sets of data are obtained at the same photon energy, $6 \mathrm{eV}$ above the Ar 1s threshold (3206.26 eV). The top curve shows photoelectron-ion coincidence measurements, and the bottom one photon-ion coincidence ones. While the top curve includes all events following photoelectron emission, the bottom curve "filters" the events in which at least one step is photon emission. Since argon is a relatively heavy atom, it is well known that after 1s 
ionization there is a branching between radiative and nonradiative decay, with the photon emission contributing by $10 \%$ to the total relaxation.

It is immediately evident that the main difference resides in the relative intensity of the peaks corresponding to the same ionic state. Whereas the main ion produced after core $1 \mathrm{~s}$ ionization in argon is $\mathrm{Ar}^{4+}$, with a branching ratio of $46.3 \%$, ion production via radiative decay leads mainly to $\mathrm{Ar}^{2+}$, with a branching ratio of $78.3 \%$. In particular, the two lower ionic charges, $\mathrm{Ar}^{+}$and $\mathrm{Ar}^{2+}$, are much more prominent in the photon-ion coincidence measurements.

The reason for such differences should be searched in the different decay pathways open after photoelectron emission. In the radiative channel, we can distinguish two main pathways, denominated $\mathrm{K} \alpha$ and $\mathrm{K} \beta$. In $\mathrm{K} \beta$, a valence electron fills the core hole and a photon is emitted:

$\gamma+\operatorname{Ar} \rightarrow \mathrm{e}_{\mathrm{ph}}+\operatorname{Ar}^{+} *\left(1 \mathrm{~s}^{-1}\right) \rightarrow \mathrm{e}_{\mathrm{ph}}+\gamma_{\mathrm{K} \beta}+\operatorname{Ar}^{+}\left(3 \mathrm{p}^{-1}\right)$

After that, the system with a single shallow vacancy is relatively long-lived.

In $\mathrm{K} \alpha$, a $2 \mathrm{p}$ electron fills the hole, a photon is emitted, and the system left with still one core vacancy continues to decay, most likely by Auger electron emission (LMM Auger, involving the 2 p vacancy and two valence levels):

$\gamma+\operatorname{Ar} \rightarrow \mathrm{e}_{\mathrm{ph}}+\operatorname{Ar}^{+} *\left(1 \mathrm{~s}^{-1}\right) \rightarrow \mathrm{e}_{\mathrm{ph}}+\gamma_{\mathrm{K} \alpha}+\operatorname{Ar}^{+} *\left(2 \mathrm{p}^{-1}\right) \rightarrow \mathrm{e}_{\mathrm{ph}}+\gamma_{\mathrm{K} \alpha}+\mathrm{e}_{\mathrm{LMM}}+\mathrm{Ar}^{2+}\left(3 \mathrm{p}^{-2}\right)$

Therefore by $\mathrm{K} \beta$ only $\mathrm{Ar}^{+}$is created, whilst by $\mathrm{K} \mathrm{Ar}^{2+}$ can be created. This situation is clearly reflected in the bottom curve of Figure 43: the much higher percentage of lowercharge ionic states compared to the top curve is due to decay patterns in which at least one step is photon emission.

(a)

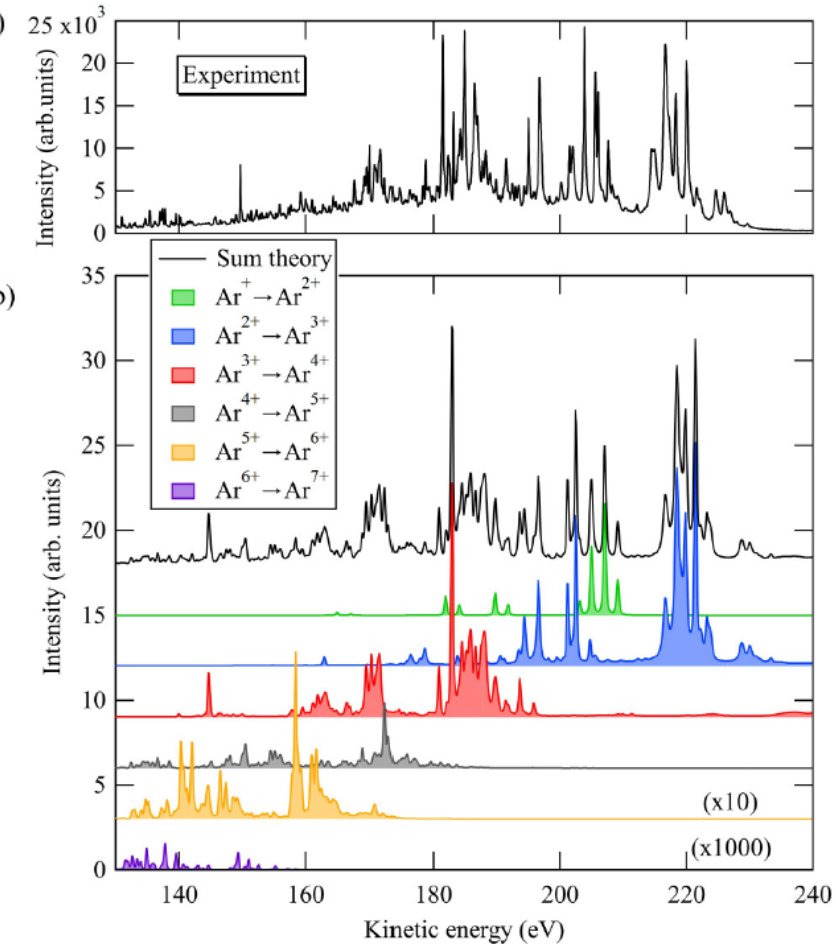

Fig.44. $L_{2,3} M M$ Auger decay after core $1 s$ ionization. (a) Experimental spectrum measured 20 eV above threshold. (b) Total calculated spectrum and partial $\mathrm{Ar}^{n^{+}} \rightarrow \mathrm{Ar}^{m^{+}}$contributions (from [74], reproduced with permission). 
To complement the coincidence experiments, the $\mathrm{L}_{2,3} \mathrm{MM}$ spectrum $20 \mathrm{eV}$ above the $1 \mathrm{~s}$ ionization threshold was measured with HAXPES. In Figure 44 we show experimental [Fig. 44(a)] and calculated LMM Auger spectra [Fig. 44(b)].

This spectrum illustrates the complexity of the LMM Auger spectrum after 1s photoionization in argon, where multistep processes dominate the Auger decay. Since the spectrum is taken in non-coincidence mode, it includes all decay cascades involving both radiative and nonradiative pathways.

The detailed assignment is presented in [74]. Here we wish to point out the possibility of regrouping the various spectral contributions in terms of transitions between ionic states, i.e.,

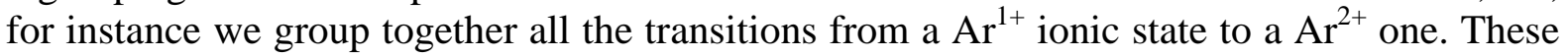
groups correspond to the subspectra shown in different colors in Fig. 44(b).

The partial $\mathrm{L}_{2,3} \mathrm{MM}$ Auger spectra obtained allow clarifying the relaxation pathways leading to the ion production shown in Fig. 43. In Table 2 we show the results of the detailed assignment for each transition from Ar ionic states with $n \rightarrow n+1$ charge $(n=1-7)$. The complexity of the LMM decay is clearly illustrated.

Similar results (not shown) are reported in [74] after resonant excitation of the type $\mathrm{Ar}$ $1 \mathrm{~s} \rightarrow 4 \mathrm{p}$ and $1 \mathrm{~s} \rightarrow 5 \mathrm{p}$. Compared to the results obtained above ionization threshold, no relevant differences in the electronic states are found, apart from the obvious presence of a spectator electron in the $4 p$ or $5 p$ orbital.

\begin{tabular}{|c|c|c|c|}
\hline Transition & $\begin{array}{c}\text { Initial } \\
\text { configuration }\end{array}$ & $\begin{array}{c}\text { Final } \\
\text { configuration }\end{array}$ & $\begin{array}{c}\text { Kinetic } \\
\text { energy }(\mathrm{eV})\end{array}$ \\
\hline \multirow[t]{3}{*}{$\mathrm{Ar}^{+} \rightarrow \mathrm{Ar}^{2+}$} & $2 p^{5}$ & $3 p^{4}$ & $202-210$ \\
\hline & $2 p^{5}$ & $3 s^{1} 3 p^{5}$ & 180-194 \\
\hline & $2 p^{5}$ & $3 s^{0}$ & $164-168$ \\
\hline \multirow[t]{6}{*}{$\mathrm{Ar}^{2+} \rightarrow \mathrm{Ar}^{3+}$} & $2 p^{4}$ & $2 p^{5} 3 p^{4}$ & $215-235$ \\
\hline & $2 p^{5} 3 p^{5}$ & $3 p^{3}$ & $198-208$ \\
\hline & $2 p^{4}$ & $2 p^{5} 3 s^{1} 3 p^{5}$ & $190-198$ \\
\hline & $2 p^{5} 3 p^{5}$ & $3 s^{1} 3 p^{4}$ & $182-190$ \\
\hline & $2 p^{4}$ & $2 p^{5} 3 s^{0}$ & $172-182$ \\
\hline & $2 p^{5} 3 p^{5}$ & $3 s^{0} 3 p^{5}$ & $159-164$ \\
\hline \multirow[t]{6}{*}{$\mathrm{Ar}^{3+} \rightarrow \mathrm{Ar}^{4+}$} & $2 s^{1} 3 p^{4}$ & $3 s^{1} 3 p^{3}$ & $230-240$ \\
\hline & $2 s^{1} 3 p^{4}$ & $3 s^{0} 3 p^{4}$ & $215-230$ \\
\hline & $2 s^{1} 2 p^{5} 3 p^{5}$ & $2 s^{1} 3 p^{3}$ & $200-215$ \\
\hline & $2 p^{5} 3 p^{4}$ & $3 p^{2}$ & $178-197$ \\
\hline & $2 p^{5} 3 p^{4}$ & $3 s^{1} 3 p^{3}$ & $155-178$ \\
\hline & $2 p^{5} 3 p^{4}$ & $3 s^{0} 3 p^{4}$ & $139-153$ \\
\hline \multirow[t]{4}{*}{$\mathrm{Ar}^{4+} \rightarrow \mathrm{Ar}^{5+}$} & $2 p^{5} 3 s^{1} 3 p^{4}$ & $3 s^{1} 3 p^{2}$ & $168-190$ \\
\hline & $2 p^{5} 3 p^{3}$ & $3 s^{1} 3 p^{2}$ & $158-168$ \\
\hline & $2 p^{5} 3 s^{1} 3 p^{4}$ & $3 s^{0} 3 p^{3}$ & $140-158$ \\
\hline & $2 p^{5} 3 p^{3}$ & $3 s^{0} 3 p^{3}$ & $130-140$ \\
\hline \multirow[t]{5}{*}{$\mathrm{Ar}^{5+} \rightarrow \mathrm{Ar}^{6+}$} & $2 p^{5} 3 s^{1} 3 p^{3}$ & $3 s^{1} 3 p^{1}$ & $160-180$ \\
\hline & $2 p^{5} 3 p^{2}$ & $3 s^{2}$ & $157-160$ \\
\hline & $2 p^{5} 3 p^{2}$ & $3 s^{1} 3 p^{1}$ & $136-155$ \\
\hline & $2 p^{5} 3 s^{1} 3 p^{3}$ & $3 s^{0} 3 p^{2}$ & $136-155$ \\
\hline & $2 p^{5} 3 p^{2}$ & $3 s^{1} 3 p^{1}$ & $132-136$ \\
\hline $\mathrm{Ar}^{6+} \rightarrow \mathrm{Ar}^{7+}$ & $2 p^{5} 3 s^{1} 3 p^{2}$ & $3 s^{1} 3 p^{0}+3 s^{0} 3 p^{1}$ & $130-160$ \\
\hline
\end{tabular}

Table 2. Electronic configurations of initial and final electronic states in the calculated $L_{2,3} M M$ Auger decay after argon 1 s core ionization (from [74], reproduced with permission). 


\subsection{Core-hole localization/delocalization}

Core levels in molecules are usually considered highly localized around one particular atomic site. However, in case of symmetric molecules with two chemically equivalent atoms, a debate exists in the literature on whether when a core hole is created by photoionization it is localized or delocalized over the two equivalent sites ([75] and references therein).

In an experimental and theoretical study of a prototypical system with two equivalent atoms, namely core-ionized carbon disulphide $\left(\mathrm{CS}_{2}\right)$, we have shown that it is feasible to distinguish between molecular fragmentation pathways in which the core hole can be assumed to be either localized on one sulphur atom or delocalized between the two equivalent sulphur atoms. The experimental technique used was to obtain photoelectron angular distributions within the molecular frame (see [75] for experimental details).

We were able to directly probe entanglement or disentanglement of quantum pathways as a function of how the molecule dissociates. Furthermore, a conceptual analogy with the iconic Schrödinger's cat thought experiment is established. In the present work, a molecular equivalent to this thought experiment is studied. Two states of equal probability are created by ionization of the $S$ 1s core level in carbon disulphide, $\mathrm{CS}_{2}$. After photoionization, the system emits Auger electrons, and dissociates into several ions.

Several of these charged particles were measured in coincidence mode. In some pathways, a specific fragmentation pattern points out at which sulphur atom was initially ionized. Therefore, the core hole is localized. Other fragmentation patterns cannot distinguish between the sulphur atoms, and therefore the core hole is delocalized. This selectivity is demonstrated by obtaining MFPADs (molecular-frame photoelectron angular distributions.

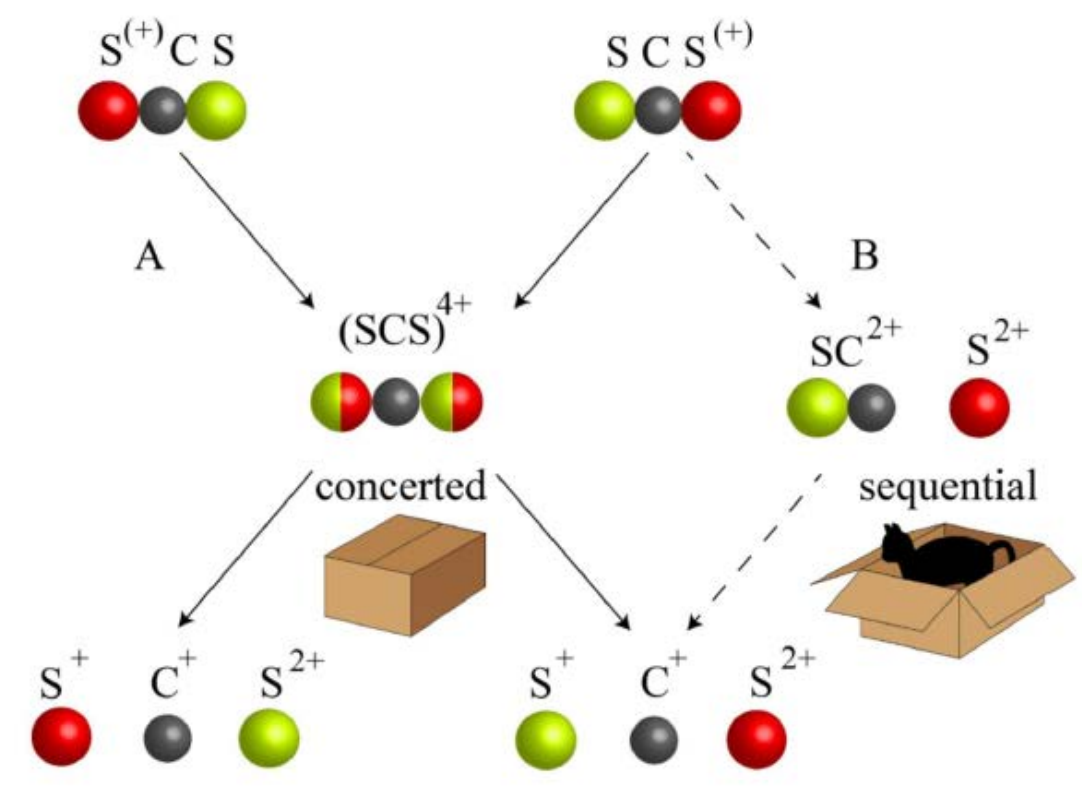

Fig.45. Top: $\mathrm{CS}_{2}{ }^{+}$ions created by S 1s photoionization of $\mathrm{CS}_{2}$ at 2,488 eV photon energy. The site of the initial $\mathrm{S}$ 1s core hole is indicated in red (left, 1sL; right, 1sR). Middle: intermediate multiply charged cations created by Auger decay of the $\mathrm{S} 1 \mathrm{~s}$ core hole. On the left is a $\mathrm{CS}_{2}{ }^{4+}$ ion produced when charge transfer is faster than molecular fragmentation. On the right, fragmentation competes with charge transfer, leading to a partially fragmented intermediate state. Bottom: final fully fragmented state, with the $\mathrm{S}^{2+}$ ion detected to the right. The final state is reached in two distinct ways: (1) concerted fragmentation of $\mathrm{CS}_{2}{ }^{4+}$ (Case A, solid arrows), or (2) sequential fragmentation of the molecule (Case $\mathrm{B}$, dashed arrows. The opened and unopened boxes (chambers) refer to the analogy to Schrödinger's cat (from [75], reproduced with permission). 
In Figure 45 we show the heart of the experiment.

The starting point is the core-ionized $\mathrm{CS}_{2}{ }^{+}$molecule (top). During Auger decay and subsequent molecular fragmentation, different pathways can lead to $\mathrm{S}^{+}+\mathrm{C}^{+}+\mathrm{S}^{2+}$ (bottom).

The electronic decay starts with KLL Auger decay on the ionized sulphur atom, followed by charge redistribution and asymmetric fragmentation. Such process can lead either to a symmetrical multiply ionized cation before dissociation, or to an asymmetrical charge distribution specific to the initial core-hole site.

In Figure 45, two different decay pathways can be identified. Their difference depends on whether there is charge transfer in the $\mathrm{CS}_{2}{ }^{+4}$ ion before it dissociates. In the pathway A (solid lines), the Auger decay produces a symmetric $\mathrm{CS}_{2}{ }^{+4}$ cation, with equal decay probabilities from either $S$ atom. In this case, charge redistribution occurs faster than molecular fragmentation, and the dissociation must be concerted: the three atomic cations are ejected at the same time in a Coulomb explosion. Therefore, the information concerning from which $\mathrm{S}$ atom the initial vacancy was created is lost.

Back to the analogy with the Schrödinger's cat, pathway A corresponds to a situation where the box stays unopened, and the status of the cat (dead or alive) is not revealed by observation.

In the pathway B (dotted lines in Figure 45), the molecular ion fragments before charge redistribution, the fragmentation is sequential and asymmetrical, leading to the formation of $\mathrm{CS}^{2+}+\mathrm{S}^{2+}$ and subsequently to $\mathrm{C}^{+}+\mathrm{S}^{+}+\mathrm{S}^{2+}$.

Therefore, the photoionization-fragmentation process is best described in a localized corehole framework. In the Schrödinger's cat picture, this corresponds to opening the box and experimentally observing the status of the cat.

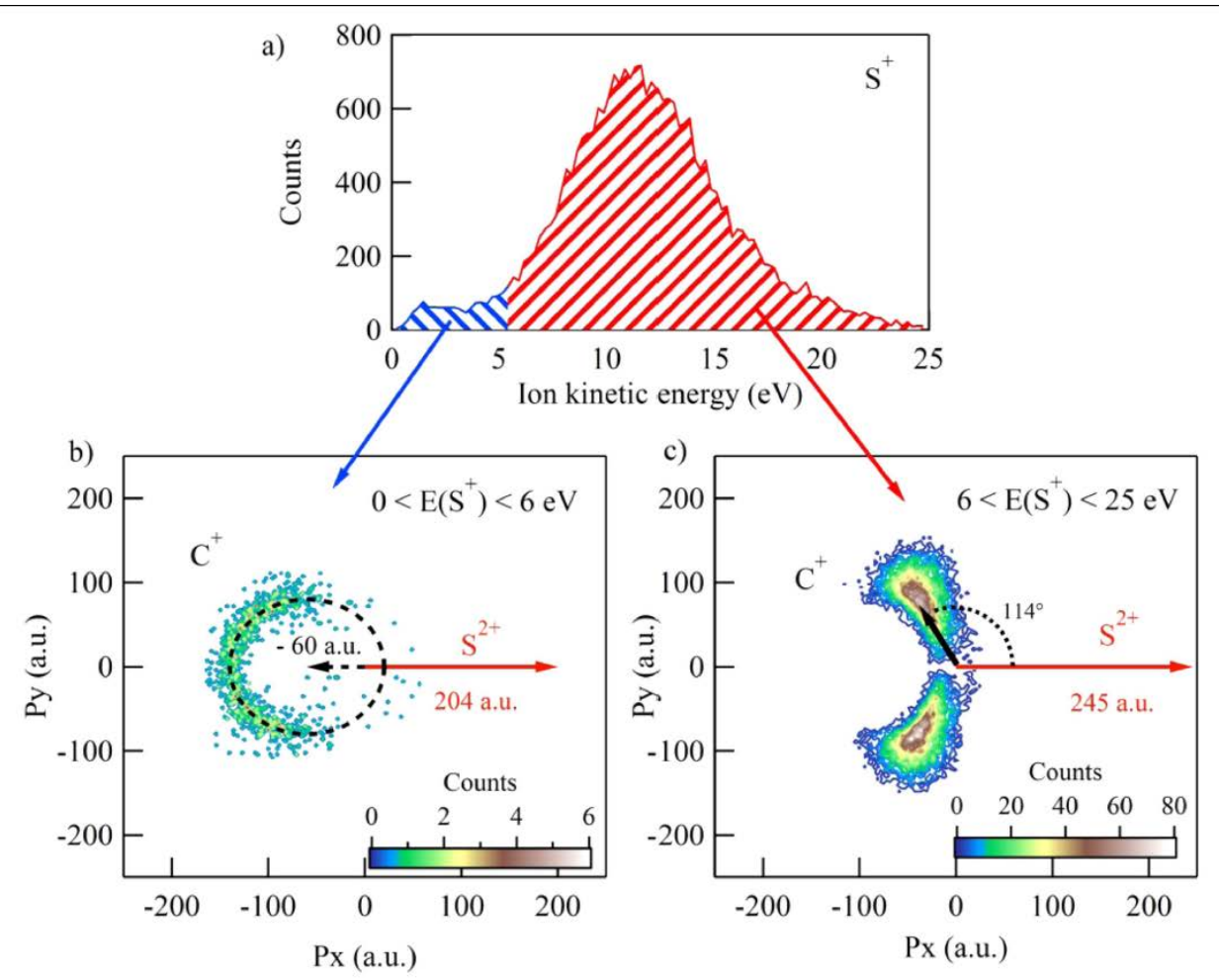

Fig.46. Selection of the fragmentation pathway. Top: (a) kinetic-energy distribution of the $\mathrm{S}^{+}$fragment in the three-body fragmentation of $\mathrm{CS}_{2}$ following $\mathrm{S} 1 \mathrm{~s}$ photoionization. Bottom: Newton diagrams showing momentum distributions of the $\mathrm{C}^{+}$fragment with respect to a fixed direction of the $\mathrm{S}^{2+}$ ion (red arrows in (b) and (c)). (b) Newton diagram associated with low kinetic-energy $\mathrm{S}^{+}$ions. (c) Newton diagram associated with high kinetic-energy $\mathrm{S}^{+}$ions (from [75], reproduced with permission). 
To confirm this model, we measured the kinetic energy distribution of the ionic fragments and derived the MFPADs.

In Figure 46 we show that concerted and sequential fragmentation of $\mathrm{CS}_{2}$ can be distinguished by filtering the events with the kinetic energy of the $\mathrm{S}^{+}$ion. The $\mathrm{S}^{+}$ions with higher kinetic energies, from 6 to $25 \mathrm{eV}$, are associated with concerted fragmentation, as illustrated by the Newton diagram in Figure 46(c), where the dissociation appears to be sudden. In contrast, $\mathrm{S}^{+}$ions with lower kinetic energies, from 0 to $6 \mathrm{eV}$, correspond to kinetic energy distributions of both $\mathrm{S}^{2+}$ and $\mathrm{C}^{+}$centred around $10 \mathrm{eV}$. For these events, $\mathrm{C}^{+}$ions gain more kinetic energy, and their momenta are distributed on a circle displaced by 60 a.u. relative to the centre of mass (Figure 46(b)). This Newton diagram illustrates that undissociated $\mathrm{CS}_{2}{ }^{+}$ions rotate with respect to $\mathrm{S}^{2+}$ before they dissociate, a clear footprint of sequential fragmentation (see [75] for more details).

To derive the MFPADs associated with each dissociative pathway, the direction of $\mathrm{S}^{2+}$ is taken as a reference to define the orientation of the molecular axis and thus the recoil frame.

MFPADs associated with concerted and sequential fragmentation are shown in Figure 47 for two orientations of the molecular axis with respect to the polarization vector of the incident radiation, together with theoretical calculations assuming either a localized or a delocalized core hole.

In case A (concerted fragmentation), the MFPADs for both molecular orientations are symmetrical with respect to the plane of symmetry of the molecule. The same characteristics are well reproduced by calculations assuming a delocalized core hole. Therefore, MFPADs measured in this case are insensitive to which sulphur atom is ionized. It is likely that rapid charge transfer occurs before dissociation, and any information on the initial site of the core hole is lost. At variance with that, for case B (sequential fragmentation), the MFPADs for both molecular orientations are quite asymmetrical with respect to the axis of the molecule, and the main structures agree very well with calculations assuming a localized core hole. The observed asymmetry is direct evidence for localization of the initial core hole on the sulphur atom ultimately detected as $\mathrm{S}^{2+}$, because the experiment selected events that lead to sequential dissociation.
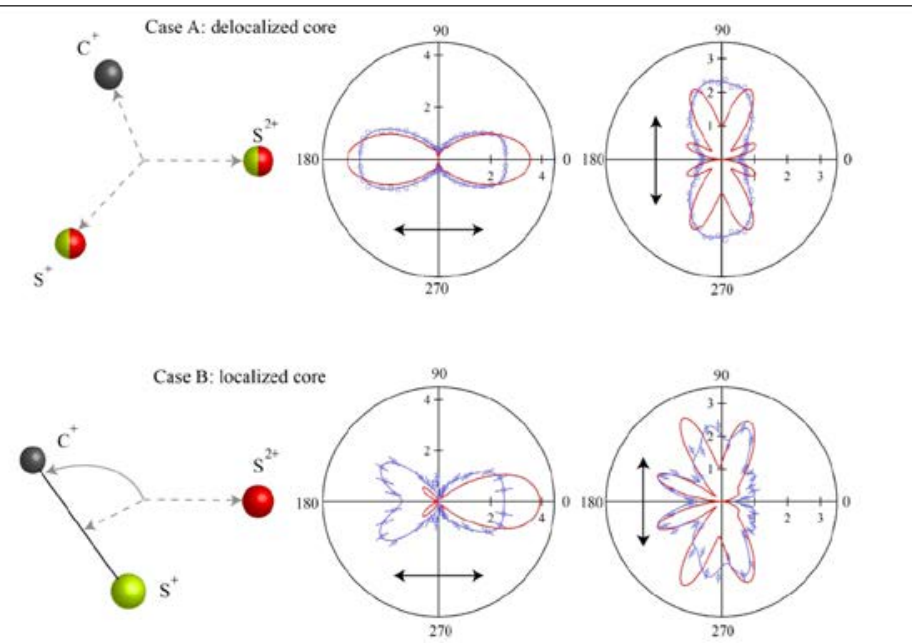

Fig.47. Polar plots of molecular-frame photoelectron angular distributions (MFPADs) obtained after photoionization of $\mathrm{CS}_{2} 10 \mathrm{eV}$ above the S 1s threshold. Top: MFPADs for concerted fragmentation (Case A). Bottom: MFPADs for sequential fragmentation (Case B). In each case, MFPADs are shown for the molecular axis oriented either parallel or perpendicular to the polarization vector of the incident radiation (black arrows) (from [75], reproduced with permission). 


\section{Perspectives}

We present here the state of the art for research in atomic and molecular physics by spectroscopic techniques in the 2-12 keV regime. We show that the new opportunities offered by the recent development of instrumentation, including both beam lines and spectrometers, has given a great impulse to the field, mainly in terms of new phenomena which could be identified. However, we would like to underline that this field is just at the beginning, and further important developments can be forecasted which shall take place in the next years.

One possible issue that we can point out is that most of the pioneering work we have reviewed here has been performed at one facility, namely SOLEIL. However, following this lead, we wish to underline that the field is expanding, and several new activities are developing at other synchrotron radiation facilities.

At SPring-8, Japan, hard x-rays up to $40 \mathrm{keV}$ are available. Recently some experiments on isolated atoms and molecules have been performed by M. Oura and the local team in collaboration with some of us on two RIKEN beam lines [76]. Xe 1s photoionization and decay [77] and I 1s photoionization and decay in $\mathrm{CH}_{3} \mathrm{I}$ and $\mathrm{CF}_{3} \mathrm{I}$ [78] were recently measured there.

At the ESRF, Grenoble, France, new experiments are in progress on Compton scattering and non-dipole effects in the hard $\mathrm{x}$-ray range, around $40 \mathrm{keV}$ [79].

In general, we can predict that the investigation on deeper core levels and shorter lifetimes will continue and expand, and new effects will be revealed. A typical example is the Compton scattering, which is basically uncharted (and challenging) territory for electron spectroscopy of isolated species.

New developments are planned by our group. Some of those concern the field of x-ray emission spectroscopies. On the GALAXIES beam line at SOLEIL there is a permanent end station for x-ray emission and RIXS measurements, and we have some preliminary results from there. Furthermore, we are building a new X-ray spectrometer, called MOSARIX, to be used at synchrotron as well as at free-electron laser sources. MOSARIX will operate in the tender $\mathrm{x}$-ray domain (2-5 keV) with high efficiency, allowing performing $\mathrm{x}$-ray emission and coincidence experiments between x-ray photons and ions or electrons. It would be the first time for such coincidences with energy-resolved photons. MOSARIX is a multi-crystal HAPG von Hamos spectrometer, equipped with a fast time and position sensitive detection system, allowing performing coincidences, or with a CCD camera. RIXS measurements with high detuning are nowadays difficult because the absorption cross section becomes very small, but those will become feasible with the new spectrometer.

Another development concerns the possibility of deriving Molecular Frame Photon Angular Distributions (MFPhADs). While MFPADs are currently obtained (see 4.10), the possibility of measuring photon angular distributions in the molecular frame is basically unexplored. This would become possible through x-ray photon - ion -ion coincidences measurements thanks to a just-built state-of-the-art x-ray photon detector with time and position capability [80] which would be coupled to an ion time-of-flight detector with position-sensitive capabilities.

Further planned progresses include a general method for observation and control of electron dynamics in large organic molecules in the energy domain. The ambition of the current project is to explore and push the potential of the core-hole clock spectroscopy towards the measurements of electron delocalization and transfer in organic molecules placed in different media in the attosecond timescale. We also plan to build an Auger electron - ion coincidence apparatus in the tender x-ray domain which will provide a valuable extension for the studies on Auger decay cascades to larger molecular systems, including molecules of biological 
interest. This project is based on an efficient electron analyser coupled to an ion time-offlight with position-sensitive detection.

Finally, a sought-after goal is to be able to induce site selective fragmentation, which has been very seldom observed in the soft x-ray domain (see e.g. [81,82]). The difficulty in systems containing light elements is that the relaxation processes after core excitation/ionization involve typically valence orbitals, which are delocalized, and therefore a really selective bond breaking is rare. At variance with that, after ionization of deep core shell the first steps of the Auger decay reach shallower orbitals which are still localized on the initial atom. We plan to take advantage of such cascade Auger effect to increase the possibility of obtaining site-selective fragmentation.

\section{Conclusion}

We have reviewed the current status of research in atomic and molecular physics by HAXPES (HArd X-ray PhotoElectron Spectroscopy) and related techniques such as Auger electron spectroscopy, x-ray emission and electron-ion coincidence measurements in the tender x-ray regime. In general, a combination of such tools can provide a deep understanding of electron and nuclear dynamics of photoexcited atoms and molecules. We show here how these techniques can be applied in a newly available photon energy range, the so-called tender $\mathrm{x}$-ray regime $(2-12 \mathrm{keV})$, and on which kind of new phenomena it is nowadays possible to shed light.

Beam lines and spectrometers providing state-of-the-art photon bandwidth and electron kinetic energy resolution have become available only in the last few years. These recent much improved experimental conditions have allowed extending to the hard x-ray range some concepts and methods already well established in soft x-ray spectroscopies. Deeper and deeper core levels are now reachable under state-of-the-art experimental conditions. Their shorter and shorter lifetime before a relaxation process occurs allows entering a dynamical regime not previously accessible. A profound knowledge of such excitation and decay processes is very important from a fundamental point of view, but it has foreseeable applications in various fields such as assessment of radiation damage in human tissues and biosystems, plasma physics and astrophysics, and many other fields outside physics.

In particular, investigations of electron and nuclear dynamics in the femtosecond and even attosecond time scale have become possible. Complex multistep processes following deepcore ionization can now be enlightened in great detail. Other phenomena, like e.g. recoilinduced effects and a new kind of ionic states with double core holes, can be observed by xray single-photon absorption and have been discussed at length. Future perspectives have been also illustrated. While the pioneering work illustrated here has been mostly performed at the French synchrotron SOLEIL, new experimental programs under development at other international facilities have also been mentioned. Future developments and challenges include e.g. the investigation of Compton scattering in isolated atoms and molecules by electron spectroscopy techniques.

\section{Acknowledgments}

The authors are grateful to the GALAXIES and LUCIA teams for a smooth running of the two facilities. In particular, the contribution from D. Céolin is acknowledged. A large collaboration has been established at SOLEIL, with many different international teams. All coworkers can be found in the reference list. Special thanks are due to R. Püttner, who has participated in most of the experiments on GALAXIES. 
[1] A. Einstein, Ann. Phys. 17, 132 (1905)

[2] K. Siegbahn, C. Nordling, G. Johansson, J. Hedman, P. F. Hedén, K. Hamrin, U. Gelius,

T. Bergmark, L. O. Werme, R. Manne, Y. Baer, ESCA Applied to Free Molecules (North Holland, Amsterdam, 1971)

[3] N. Mårtensson, S. Svensson and U. Gelius, J. Phys. B: At. Mol. Opt. Phys. 20, 6243 (1987), and references therein

[4] O. Björneholm, A. Nilsson, A. Sandell, B. Hernnäs and N. Mårtensson, Phys. Rev. Lett. 68, 1892 (1992)

[5] O. Björneholm, S. Sundin, S. Svensson, R. R. T. Marinho, A. Naves de Brito, F. Gel'mukhanov and H. Ågren, Phys. Rev. Lett. 79, 3150 (1997)

[6] P. A. Brühwiler, O. Karis and N. Mårtensson, Rev. Mod. Phys. 74, 703 (2002)

[7] M. Simon, L. Journel, R. Guillemin, W. C. Stolte, I. Minkov, F. Gel'mukhanov, P. Sałek, H. Ågren, S. Carniato, R. Taïeb, A. C. Hudson, and D. W. Lindle, Phys. Rev. A 73, 020706 (2006).

[8]I. Nenner and P. Morin, in "VUV and Soft X-Ray Photoionisation Studies in Atoms and Molecules”, eds. U. Becker and D. A. Shirley (Plenum Press, London, 1996)

[9] D. M. Hanson, Adv. Chem. Phys. 77, 1 (1990)

[10] R. Dörner, V. Mergel, O. Jagutzki, L. Spielberger, J. Ullrich, R. Moshammer and H. Schmidt-Böcking, Phys. Rep. 330, 95 (2000), and references therein

[11] https://sites.google.com/a/lbl.gov/haxpes2017/agenda

[12] M. Simon, M. N. Piancastelli and D. W. Lindle, "Hard X-Ray Photoelectron Spectroscopy from Atoms and Molecules" in "HAXPES”, ed. J. Woicik, Springer Series in Surface Science Vol. 59, Springer Heidelberg New York Dordrecht London, 2016

[13]G. S. Brown, M. H. Chen, B. Crasemann and G. E. Ice, Phys. Rev. Lett. 45, 1937 (1980)

[14] O. Björneholm, M. Bässler, I. Hjelte, R. Feifel, H. Wang, C. Miron, M. N. Piancastelli, S. Svensson, S. L. Sorensen, F. Gel'mukhanov and H. Ågren, Phys. Rev. Lett. 84, 2826 (2000)

[15] R. Feifel, F. Burmeister, P. Sałek, M. N. Piancastelli, M. Bässler, S. L. Sorensen, C. Miron, H. Wang, I. Hjelte, O. Björneholm, A. Naves de Brito, F. Gel'mukhanov, H. Ågren and S. Svensson Phys. Rev. Lett. 85, 3133 (2000)

[16] I. Hjelte, M. N. Piancastelli, R. F. Fink, O. Björneholm, M. Bässler, R. Feifel, A. Giertz, H. Wang, K. Wiesner, A. Ausmees, C. Miron, S. L. Sorensen and S. Svensson, Chem. Phys. Lett. 334, 151 (2001)

[17] L. Rosenqvist, K. Wiesner, A. Naves de Brito, M. Bässler, A. Ausmees, R. Feifel, I. Hjelte, S. L. Sorensen, C. Miron, H. Wang, M. N. Piancastelli, S. Svensson and O. Björneholm, J. Chem. Phys. 115, 3614 (2001)

[18] I. Hjelte, M. N. Piancastelli, C. M. Jansson, K. Wiesner, O. Björneholm, M. Bässler, S. L. Sorensen and S. Svensson, Chem. Phys. Lett. 370, 781 (2003)

[19] O. Travnikova, V. Kimberg, R. Flammini, X.-J. Liu, M. Patanen, C. Nicolas, S. Svensson and C. Miron, J.Phys.Chem.Lett. 4, 2361 (2013)

[20] H. Sann, T. Havermeier, C. Müller, H.-K. Kim, F. Trinter, M. Waitz, J. Voigtsberger, F. Sturm, T. Bauer, R. Wallauer, D. Schneider, M. Weller, C. Goihl, J. Tross, K. Cole, J. Wu, M. S. Schöffler, H. Schmidt-Böcking, T. Jahnke, M. Simon and R. Dörner, Phys.Rev.Lett. 117, 243002 (2016)

[21] F. Gel'mukhanov and H. Ågren, Phys. Rep. 312, 87 (1999)

[22] F. Gel'mukhanov, P. Sałek, T. Privalov, and H. Ågren, Phys. Rev. A 59, 380 (1999 
[23] G. B. Armen, T. Åberg, J. C. Levin, B. Crasemann, M. H. Chen, G. E. Ice and G. S. Brown, Phys. Rev. Lett. 54, 1142 (1985)

[24] U. Arp, T. LeBrun, S. H. Southworth, M. A. MacDonald and M. Jung, Phys.Rev.A 55, 4273 (1997), and references therein

[25] L. S. Cederbaum, F. Tarantelli, A. Sgamellotti and J. Schirmer, J. Chem. Phys. 85, 6513 (1986)

[26] D. L. Matthews, B. M. Johnson, J. J. Mackey and C. F. Moore, Phys.Rev.Lett. 31, 1331 (1973)

[27] C. W. Woods, Robert L. Kauffman, K. A. Jamison, N. Stolterfoht and P. Richard, Phys.Rev.A 12, 1393 (1975)

[28] S. Hagmann, S. Kelbch, C. L. Cocke, P. Richard, and A. Skutlartz, Phys.Rev.A 34, 2897 (1986)

[29] Ž. Šmit, M. Žitnik, L. Avaldi, R. Camilloni, E. Fainelli, A. Muhleisen and G. Stefani, Phys.Rev.A 49, 1480 (1994)

[30] J. H. D. Eland, M. Tashiro, P. Linusson, M. Ehara, K. Ueda and R. Feifel, Phys. Rev. Lett. 105, 213005(2010)

[31] M. Nakano, P. Selles, P. Lablanquie, Y. Hikosaka, F. Penent, E. Shigemasa, K. Ito and S. Carniato, Phys. Rev. Lett. 111, 123001 (2013)

[32] P. Lablanquie et al, Phys. Rev. Lett. 107,193004 (2011)

[33] M. Nakano et al, Phys. Rev. Lett. 110, 163001 (2013)

[34] P. Linusson, S. Fritzsche, J. H. D. Eland, M. Mucke and R. Feifel, Phys. Rev. A 87 043409 (2013)

[35] N. Berrah et al, Proc. Natl Acad. Sci. USA 108, 16912 (2011)

[36] P. Salén et al, Phys. Rev. Lett. 108, 153003 (2012)

[37] M. Larsson et al, J.Phys.B: At. Mol. Opt. Phys. 46, 164034 (2013)

[38] V. Zhaunerchyk et al, J.Phys.B: At. Mol. Opt. Phys. 46, 164030 (2013)

[39] L.J.Frasinski et al, Phys.Rev.Lett. 111, 073002 (2013)

[40] https://www.esrf.eu/UsersAndScience/Experiments/EMD/ID26

[41] J. M. Ablett, J. M. Dubuisson, T. Moreno, D. Céolin, E. Raimon, D. Prieur, D. Corruble, J. Coquet, A. Lestrade, C. Bourgoin and J. P. Rueff, Journal of Physics Conferences Series. 425, 052007 (2013)

[42] A.-M. Flank, G. Cauchon, P. Lagarde, S. Bac, M. Janousch, R. Wetter, J.-M. Dubuisson, M. Idir, F. Langlois, T. Moreno and D. Vantelon, Nucl. Instr. Meth. Phys. Res. B 246, 269 (2006)

[43] M. Kavčič, M. Budnar, A. Muhleisen, F. Gasser, M. Žitnik, K. Bučar, and R. Bohinc, Rev. Sci. Instrum. 83, 033113 (2012)

[44] D. Céolin, J. M. Ablett, D. Prieur, T. Moreno, J.-P. Rueff, T. Marchenko, L. Journel, R. Guillemin, B. Pilette, T. Marin and M. Simon, J. Electron Spectrosc. Relat. Phenom. 190, 188 (2013)

[45] C. Bomme, R. Guillemin , T. Marin , L. Journel , T. Marchenko , D. Dowek , N. Trcera , B. Pilette, A. Avila, H. Ringuenet, R. K. Kushawaha and M. Simon, Rev. Sci. Instrum. 84, 103104 (2013)

[46] T. Marchenko, L. Journel, T. Marin, R. Guillemin, S. Carniato, M. Žitnik, M. Kavčič, K. Bučar, A. Mihelič, J. Hoszowska, W. Cao and M. Simon, J. Chem.Phys.134, 144308 (2011)

[47] M. N. Piancastelli, G. Goldsztejn, T. Marchenko, R. Guillemin, R. K. Kushawaha, L. Journel, S. Carniato, J.-P. Rueff, D. Céolin and M. Simon, J. Phys. B: At. Mol. Opt. Phys. 47, 124031 (2014)

[48] T. Marchenko, S. Carniato, L. Journel, R. Guillemin, E. Kawerk, M. Žitnik, M. Kavčič, K. Bučar, R. Bohinc, M. Petric, V. Vaz da Cruz, F. Gel'mukhanov and M. Simon, Phys.Rev.X 5, 031021 (2015) 
[49] R. K. Kushawaha, M. Patanen, R. Guillemin, L. Journel, C. Miron, M. Simon, M. N. Piancastelli, C. Skates and P. Decleva, PNAS 110, 15201 (2013)

[50]R. Püttner, G. Goldsztejn, D. Céolin, J.-P. Rueff, T. Moreno, R. K. Kushawaha, T. Marchenko, R. Guillemin, L. Journel, D. W. Lindle, M. N. Piancastelli and M. Simon, Phys.Rev.Lett. 114, 093001 (2015)

[51] ) G. Goldsztejn, T. Marchenko, R. Püttner, L. Journel, R. Guillemin, S. Carniato, P. Selles, O. Travnikova, D. Céolin, A. Lago, R. Feifel, P. Lablanquie, M. N. Piancastelli, F. Penent and M. Simon, Phys.Rev.Lett. 117, 133001 (2016)

[52] G. Goldsztejn, R. Püttner, L. Journel, R. Guillemin, O. Travnikova, B. Cunha de Miranda, I. Ismail, S. Carniato, P. Selles, D. Céolin, A. F. Lago, R. Feifel, P. Lablanquie, F. Penent, M. N. Piancastelli, M. Simon and T. Marchenko, Phys.Rev.A 96, 012513 (2017)

[53] Y. Li, L. Liu, C. Gao, J. Zeng and J. Yuan, J.Electron Spectrosc.Relat.Phenom. 226, 26 (2018)

[54] R. Feifel, J. H. D. Eland, S. Carniato, P. Selles, R. Püttner, D. Koulentianos, T. Marchenko, L. Journel, R. Guillemin, G. Goldsztejn, O. Travnikova, I. Ismail, B. Cunha de Miranda, A. F. Lago, D. Céolin, P. Lablanquie, F. Penent, M. N. Piancastelli and M. Simon, Sci.Rep. 7, 13317 (2017)

[55] D. Koulentianos, R. Püttner, G. Goldsztejn, T. Marchenko, O. Travnikova, L. Journel, R. Guillemin, D. Céolin, M. N. Piancastelli, M. Simon and R. Feifel, Phys.Chem.Chem.Phys. 20, 2724 (2018)

[56] D. Koulentianos, S. Carniato, R. Püttner, G. Goldsztejn, T. Marchenko, O. Travnikova, L. Journel, R. Guillemin, D. Céolin, M. L. M. Rocco, M. N. Piancastelli, R. Feifel and M. Simon, J.Chem.Phys. 149, 134313 (2018)

[57] T. Marchenko, L. Inhester, G. Goldsztejn, O. Travnikova, L. Journel, R. Guillemin, I. Ismail, D. Koulentianos, D. Céolin, R. Püttner, M. N. Piancastelli and M. Simon, Phys.Rev.A 98, 063403 (2018)

[58] S. Carniato, P. Selles, P. Lablanquie, J. Palaudoux, L. Andric, M. Nakano, Y. Hikosaka, K. Ito, M. Simon, G. Goldsztejn, T. Marchenko, L. Journel, R. Guillemin, O. Travnikova, D. Céolin, M. N. Piancastelli and F. Penent, Phys.Rev.A 94, 013416 (2016)

[59] T. Marchenko, G. Goldsztejn, K. Jänkälä, O. Travnikova, L. Journel, R. Guillemin, N. Sisourat, D. Céolin, M. Žitnik, M. Kavčič, K. Bučar, A. Mihelič, B. Cunha de Miranda, I. Ismail, A. F. Lago, F. Gel'mukhanov, R. Püttner, M. N. Piancastelli and M. Simon, Phys.Rev.Lett 119, 133001 (2017)

[60] O.Travnikova, T. Marchenko, G. Goldsztejn, K. Jänkälä, N. Sisourat, S. Carniato, R. Guillemin, L. Journel, D. Céolin, R. Püttner, H. Iwayama, E. Shigemasa, M. N. Piancastelli and M. Simon, Phys.Rev.Lett. 116, 213001 (2016), and references therein

[61] O.Travnikova, N.Sisourat, T. Marchenko, G. Goldsztejn, R. Guillemin, L. Journel, D. Céolin, I. Ismail, A. Lago, R. Püttner, M. N. Piancastelli and M. Simon, Phys.Rev.Lett. 118, 213001 (2017)

[62] M. Simon, R. Püttner, T. Marchenko, R. Guillemin, R. K. Kushawaha, L. Journel, G. Goldsztejn, M. N. Piancastelli, J. M. Ablett, J.-P. Rueff and D. Céolin, Nature Comm. 5, 4069 (2014)

[63] E. Kukk, T. D. Thomas, K. Ueda, D. Céolin, S. Granroth, K. Kooser, O. Travnikova, D. Iablonsky, P. Decleva, D. Ayuso, R. Püttner, H. Levola, G. Goldsztejn, T. Marchenko, M. N. Piancastelli and M. Simon, Phys.Rev.A 95, 042509 (2017), and references therein

[64] E. Kukk, T. D. Thomas, D. Céolin, S. Granroth, O. Travnikova, M. Berholts, T. Marchenko, R. Guillemin, L. Journel, I. Ismail, R. Püttner, M. N. Piancastelli, K. Ueda and M. Simon, Phys.Rev.Lett. 121, 073002 (2018)

[65] M. Žitnik et al, Phys. Rev. A 93, 021401(R) (2016) 
[66] D. Céolin, T. Marchenko, R. Guillemin, L. Journel, R. K. Kushawaha, S. Carniato, S.-M Huttula, J.-P. Rueff, G.B. Armen, M.N. Piancastelli and M. Simon, Phys.Rev.A 91, 022502 (2015)

[67] M. Neeb, J.-E. Rubensson, M. Biermann and W. Eberhardt, J. Electron Spectrosc. Relat. Phenom. 67, 261(1994)

[68] M. N. Piancastelli, J. Electron Spectrosc. Relat. Phenom. 107, 1 (2000) and references therein

[69] R. K. Kushawaha, K.Jänkälä, T. Marchenko, G. Goldsztejn, R. Guillemin, L. Journel, D. Céolin, J.-P. Rueff, A. F. Lago, R. Püttner, M. N. Piancastelli and M. Simon, Phys.Rev.A 92, 013427 (2015)

[70] G. Goldsztejn, T. Marchenko, R. Püttner, L. Journel, R. Guillemin, O. Travnikova, R. K. Kushawaha, B. Cunha de Miranda, I. Ismail, D. Céolin, M. N. Piancastelli and M. Simon, Phys.Rev.A 95, 012509 (2017)

[71] G. Goldsztejn, T. Marchenko, D. Céolin, L. Journel, R. Guillemin, J.-P. Rueff, R. K. Kushawaha, R. Püttner, M. N. Piancastelli and M. Simon, Phys.Chem.Chem.Phys. 18, 15133 (2016)

[72] R. Guillemin, S. Sheinerman, T. Marchenko, G. Goldsztejn, L. Journel, D. Céolin, R. K. Kushawaha, M.N. Piancastelli and M. Simon, Phys.Rev.A 92, 012503 (2015)

[73] R. Guillemin, S. Sheinerman, C. Bomme, T. Marin, L. Journel, T. Marchenko, R. K. Kushawaha, N. Trcera, M. N. Piancastelli and M. Simon, Phys.Rev.Lett. 109, 013001 (2012)

[74] R. Guillemin, K. Jänkälä, B. Cunha de Miranda, T. Marin, L. Journel, T. Marchenko, O. Travnikova, G. Goldsztejn, I. Ismail, R. Püttner, D. Céolin, B. Lassalle-Kaiser, M. N. Piancastelli and M. Simon, Phys. Rev.A 97, 013418 (2018)

[75] R. Guillemin, P. Decleva, M. Stener, C. Bomme, T. Marin, L. Journel, T. Marchenko, R. K. Kushawaha, K. Jänkälä, N. Trcera, K. P. Bowen, D. W. Lindle, M. N. Piancastelli and M. Simon, Nature Comm. 6, 6166 (2015)

[76] M. Oura, T. Gejo, K. Nagaya, Y. Kohmura, K. Tamasaku, L. Journel, M. N. Piancastelli and M. Simon, New J. Phys.21, 043015 (2019)

[77] M. N. Piancastelli, K. Jänkälä, L. Journel, T. Gejo, Y. Kohmura, M. Huttula, M. Simon, and M. Oura, Phys.Rev.A 95, 061402(R) (2017)

[78] N. Boudjemia, K. Jänkälä, T. Gejo, K. Nagaya, K. Tamasaku, M. Huttula, M. N. Piancastelli, M. Simon and M. Oura, Phys.Chem.Chem.Phys. 21, 5448 (2019)

[79] https://journals.aps.org/prl/accepted/45075YbaT2b1f273f3206dd5b6a9e2d4af340d701

[80] I. Ismail, R. Guillemin, T. Marchenko, O. Travnikova, J. M. Ablett, J.-P. Rueff, M. N. Piancastelli, M. Simon and L. Journel, Rev.Scient.Instrum. 89, 063107 (2018)

[81] H. Schmelz et al, J. Chem. Phys. 101, 3742 (1994)

[82] I Nenner et al, Zeitschrift für Physik D 195, 43 (1996) 